\title{
Isogeometric Unstructured Tetrahedral and Mixed-Element Bernstein-Bézier Discretizations
}

\author{
Luke Engvall, John A. Evans \\ Aerospace Engineering Sciences, University of Colorado at Boulder
}

February 15, 2017

\begin{abstract}
A well-known problem in the field of isogeometric analysis is that of surface-tovolume parameterization. In CAD packages, solid objects are represented by a collection of NURBS or T-spline surfaces, but to perform engineering analysis for many real world problems, we must find a way to parameterize the volumes of these objects as well. This has proven to be difficult using traditional isogeometric methods, as the tensor-product nature of trivariate NURBS and T-splines limit their ability to provide analysis suitable parameterizations of arbitrarily complex volumes. To overcome the limitations of trivariate NURBS and T-splines, we propose the use of unstructured Bernstein-Bézier discretizations. In earlier work, we demonstrated the feasibility of this approach in two dimensions through the construction of an automatic mesh generation environment capable of generating geometrically exact unstructured meshes comprised of rational Bézier triangles. This paper extends the concepts presented in our previous work to unstructured tetrahedral and mixed-element meshes in three dimensions. The main contributions of this paper are three-fold. First, we present a framework for creating geometrically exact meshes comprised of rational Bézier hexahedra, tetrahedra, wedges and pyramids through degree elevation of suitable linear meshes. We additionally discuss how our approach may be applied to higher-order mesh generation for more traditional finite element approaches. Next, we propose two quality metrics for threedimensional curvilinear meshes, and discuss some important considerations for mesh quality of higher-order meshes. Finally, we demonstrate the analysis suitability of the meshes constructed using our framework through numerical examples.
\end{abstract}

(C) 2016. This manuscript version is made available under the Elsevier user license http://www.elsevier.com/open-access/userlicense/1.0/ 


\section{Contents}

1 Introduction 3

2 CAD Objects 6

2.1 NURBS Objects . . . . . . . . . . . . . . . . . . . . . . . . 6

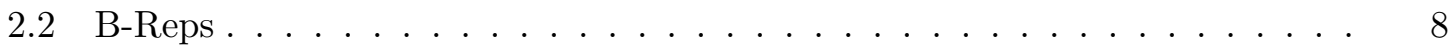

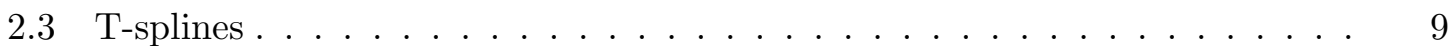

3 Meshing Geometric Primitives $\quad 13$

3.1 The Bernstein-Bézier Form . . . . . . . . . . . . . . . . . . . . . 13

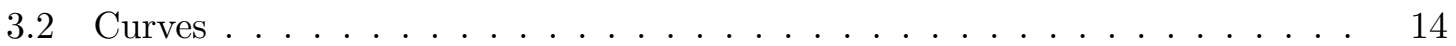

3.3 Quadrilaterals . . . . . . . . . . . . . . . . . . . . . 14

3.4 Triangles . . . . . . . . . . . . . . . . . . . . . . . 15

3.5 Hexahedra . . . . . . . . . . . . . . . . . . . . 17

3.6 Tetrahedra . . . . . . . . . . . . . . . . . 17

3.7 Wedges . . . . . . . . . . . . . . . . . . . 18

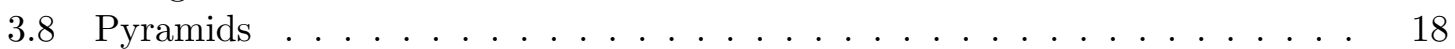

4 Bézier Extraction and Bézier Projection $\quad 19$

4.1 Bézier Extraction . . . . . . . . . . . . . . . . . . . . . 21

4.2 Bézier Projection . . . . . . . . . . . . . . . . . 22

5 Geometric Polynomial Complexity 24

6 Higher Order Mesh Construction $\quad 27$

6.1 Generation of a Compatible Linear Mesh . . . . . . . . . . . . . . . . 28

6.2 Degree Elevation of the Linear Mesh . . . . . . . . . . . . . . . . . 29

6.3 Surface Reconstruction . . . . . . . . . . . . . . . . . . 30

6.4 Smoothing of Control Points and Weights . . . . . . . . . . . . 33

6.5 Applications to Higher-Order FEA . . . . . . . . . . . . . . . . . 34

6.6 Construction of Structured Surface Meshes _ . . . . . . . . . . . . . . 35

7 Element/Mesh Refinement $\quad 36$

8 Mesh Gallery $\quad 40$

9 Mesh Quality $\quad 44$

$\begin{array}{ll}10 \text { Numerical Examples } & 48\end{array}$

10.1 Patch Test . . . . . . . . . . . . . . . . . . . . 48

10.2 Method of Manufactured Solutions . . . . . . . . . . . . . 51

10.3 Practical Examples . . . . . . . . . . . . . . . . . . 51

11 Conclusions $\quad 53$

12 Acknowledgements $\quad 54$ 


\section{Introduction}

Isogeometric analysis (IGA) was proposed as a means to remove the design-to-analysis bottleneck inherent in the traditional Computer Aided to Design (CAD) to Finite Element Analysis (FEA) design cycle [16]. By using the NURBS basis functions native to CAD packages as the basis for finite element analysis, IGA serves to provide a seamless transition between design (CAD) and analysis (FEA). However, in CAD packages, solids objects are usually represented by a collection of bounding surfaces, commonly referred to as Boundary representations or B-reps, or through a Boolean combination of geometric primitives, a process known as Constructive Solid Geometry (CSG). While these representations are adequate for providing a visual representation of objects, they do not provide an explicit parameterization of the interior volume. Unfortunately, an explicit parametrization of the interior volume is required to perform FEA for a wide array of engineering applications involving structural mechanics, fluid dynamics, and electromagnetics. Thus arises the problem of surface-to-volume parameterization, which for our purposes, is defined as follows:

Given a NURBS or T-spline surface from CAD, automatically parameterize the corresponding volume such that:

1. The parameterization is explicit.

2. The parameterization exactly matches the geometry.

3. The parameterization is analysis suitable. ${ }^{1}$

4. The parameterization is defined by piecewise polynomial or rational basis functions that satisfy linear independence, partition of unity, positivity, and higher-order continuity.

There exists a large body of work addressing this singular issue, and a wide array of numerical methods have been developed to create volume parameterizations using trivariate B-splines [25, 43], NURBS [1, 3], and T-splines [11, 12, 22, 23, 39]. Additionally, some researchers have investigated the use of unstructured Bernstein-Bézier discretizations for volume completion [13,44]. In particular, Xia and Qian have succeeded in creating geometrically exact, $C^{r}$-continuous discretizations using Bernstein-Bézier tetrahedra [41]. It should be noted, though, that $C^{1}$-continuous tetrahedral discretizations require the use of either nonic tetrahedra [45], or sextic tetrahedral macro-elements [18]. Moreover, achieving $C^{2}$-and-higher continuity requires the use of even higher order bases.

In our previous paper, we provide an extensive literature review of the state of the art of surface-to-volume parameterization for isogeometric analysis [10], and we include here a table that briefly compares each respective method. Specifically, we evaluate each method based on how well it accomplishes the goals of surface-to-volume parameterization as outlined above, based on four criteria:

1. Automatic: Is the method capable of automatically creating a volumetric parameterization from a CAD surface without user interaction?

2. Arbitrary and Complex Geometries: Is the method capable of parameterizing complex geometries, such as arbitrary genus objects, and objects with large variations in length scales?

\footnotetext{
${ }^{1}$ We say a parameterization is analysis suitable if it is both invertible and well-conditioned. A parameterization is invertible if the element-wise Jacobian is bounded from both above and below. A parameterization is well-conditioned if it preserves optimal convergence rates.
} 
3. Exact Geometry: Is the method capable of creating a geometrically exact parameterization of engineering geometries, including conic sections?

4. Analysis Suitable: Is it demonstrated that the method produces analysis suitable geometries?

For each method and each criteria, we say that the method either (a) fulfills the criteria $(\checkmark),($ b) does not fulfill the criteria $(\times)$, or $(c)$ fulfills the criteria with some caveats $(\sim)$. Of course, this ternary classification system does obscure many of the nuances of each individual method. Additionally, terminology such as "automatic" and "complex geometry" is somewhat vague, and can vary from application to application. As such, Table 1 should be used as a quick visual reference to compare various methods, and interested readers are directed to the original works for more information.

Table 1: Comparison of existing surface-to-volume approaches.

\begin{tabular}{|l|c|c|c|c|}
\hline & Automatic & $\begin{array}{l}\text { Arbitrary } \\
\text { and } \\
\text { Complex } \\
\text { Geometries }\end{array}$ & $\begin{array}{l}\text { Exact } \\
\text { Geometry }\end{array}$ & $\begin{array}{c}\text { Analysis } \\
\text { Suitable }\end{array}$ \\
\hline Swept Volumes [1] & $\times$ & $\times$ & $\checkmark$ & $\checkmark$ \\
\hline NURBS from B-Reps [3] & $\checkmark$ & $\sim$ & $\times$ & $\sim$ \\
\hline Trivariate B-splines [25] & $\sim$ & $\times$ & $\times$ & $\checkmark$ \\
\hline Multi-Block Domain [43] & $\sim$ & $\times$ & $\times$ & $\checkmark$ \\
\hline $\begin{array}{l}\text { Hybrid B-spline/Tetrahedral Dis- } \\
\text { cretizations [44] }\end{array}$ & $\sim$ & $\sim$ & $\checkmark$ & $\sim$ \\
\hline Meccano Method [12, 11] & $\checkmark$ & $\times$ & $\checkmark$ & $\checkmark$ \\
\hline $\begin{array}{l}\text { T-splines from Boundary } \\
\text { Triangulations [39] }\end{array}$ & $\checkmark$ & $\times$ & $\checkmark$ & $\checkmark$ \\
\hline $\begin{array}{l}\text { T-splines From Boolean } \\
\text { Operators [22] }\end{array}$ & $\sim$ & $\sim$ & $\checkmark$ & $\checkmark$ \\
\hline Skeleton Based Polycubes [23] & & & & \\
\hline
\end{tabular}

From the review of the available literature, it is our opinion that none of the previous work fully addresses the problem of surface-to-volume parameterization as it is stated above. In this work, we propose to address this problem through the use of unstructured BernsteinBézier discretizations. The primary motivation for considering unstructured BernsteinBézier discretizations comes from the flexibility and robustness of state-of-the-art unstructured mesh generation approaches. Unstructured tetrahedral mesh generation based on NURBS is a well established technology with many commercial and open source software options available $[14,36]$. Additionally, mixed element mesh generation has been a research topic of great interest recently, with a focus on generating hexahedral dominant meshes $[5,37]$, as well as generating boundary layer meshes for fluid flow simulations [15, 26, 29]. By employing Bézier geometric primitives, our approach combines the robustness of unstructured mesh generation with the advantageous properties of IGA, namely geometric exactness and a tight link between CAD and FEA.We do note that our approach does limit the basis to $C^{0}$-continuity on element boundaries. However, we believe that for many 
problems of practical interest, the loss of the higher-order continuous basis is an acceptable sacrifice for the both the geometric flexibility provided by unstructured discretizations, as well as the ease of implementation of our approach. Moreover, it is possible to combine our approach to surface-to-volume parameterization with the $C^{r}$-continuous tetrahedra of Xia and Qian to recover higher continuity, though this requires the use of relatively high order elements, and is limited to purely tetrahedral meshes.

In this work, we will focus our efforts on the creation of geometrically exact tetrahedral and mixed-element discretizations, as well as demonstrating their analysis suitability by example for a few practical applications. In our previous paper, we considered the analogous two-dimensional problem of area-to-volume parameterization as a proof-of-concept. We demonstrated the feasibility of this approach by developing meshing routines capable of automatically generating geometrically exact meshes composed of Bézier triangles. We also demonstrated that these meshes were suitable for isogeometric analysis. In the present work, we extend our prior work to three dimensions by:

1. Presenting a framework for creating geometrically exact tetrahedral and mixed-element Bernstein-Bézier discretizations for solid volumes in three dimensions.

2. Introducing quality metrics for higher-order curvilinear meshes.

3. Demonstrating the analysis suitability of mixed element Bernstein-Bézier discretizations through numerical examples.

We do note that the analysis suitability of mixed-element tetrahedral-hexahedral-pyramidal Bernstein-Bézier discretizations has already been demonstrated from a theoretical point of view, and it has also been shown that these disctretizations benefit from good computational efficiency [2]. However, the previous work is somewhat limited in scope, and does not consider the creation of curvilinear discretization to match complex engineering geometries.

It should further be mentioned that our approach for mesh generation is quite similar to existing curvilinear mesh generation approaches [17, 24, 28, 33, 34, 42]. However, besides the fact that our approach preserves exact geometry, there are two more key differences that should be highlighted. First of all, our approach employs variational projection for surface fitting while most existing mesh generation approaches employ point interpolation. Consequently, our approach is not subject to the issue of instability, including the presence of spurious surface oscillations, that may result from a poor choice of interpolation nodes [40]. Second of all, our approach employs a Bernstein-Bézier parametrization of the surface geometry as opposed to a Lagrange parametrization. This allows us to define necessary conditions for ensuring higher-order mesh quality, as seen in our earlier work [27].

The rest of this paper is organized as follows. In Section 2 we provide background on how surfaces are represented in CAD packages, with a particular focus on geometric modeling using NURBS, B-Reps and T-splines. Section 3 then presents the Bernstein-Bézier forms of the four geometric primitives most commonly used in unstructured mixed-element mesh generation: tetrahedra, wedges, hexahedra, and pyramids. We present both polynomial and rational Bernstein-Bézier forms with complex engineering geometries in mind. We then present some relevant mathematical preliminaries in Sections 4 and 5. With this background established, we move on to the main contribution of this work and present our procedure for mesh construction in Section 6, as well as our method for mesh refinement in Section 
7. Section 8 provides a gallery of meshes produced by our mesh generation procedure, and Section 9 touches upon some important mesh quality considerations. Finally, we conclude the paper in Section 10 with some numerical examples demonstrating the analysis suitability of the discretizations produced by our mesh construction framework.

\section{CAD Objects}

Before discussing our approach to surface-to-volume parameterization, it is useful to provide an overview of the manner in which physical objects are represented in CAD packages. We begin this section by discussing the construction of NURBS curves and surfaces. Because these objects share largely the same construction, we will collectively refer to them

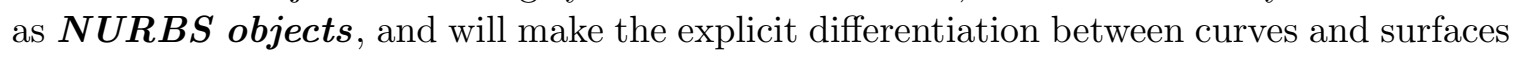
where necessary. We then present an introduction to B-Reps, which are one of the de-facto standards for representing complex geometries in modern CAD packages ${ }^{2}$. Finally, we discuss some of the limitations of these representations, and discuss T-splines as an attractive alternative.

\section{$2.1 \quad$ NURBS Objects}

NURBS (Non-Uniform Rational B-spline) objects lie at the heart of modern CAD packages due to their flexibility in representing a wide variety of geometries. The simplest NURBS object is a B-spline curve. To define a B-spline curve of polynomial degree $p$, we require:

1. A set of basis functions defined in $d_{r}$-dimensional parameter space ${ }^{3}$. This is given by a set of $n$ univariate $\left(d_{r}=1\right)$ B-spline basis functions of degree $p,\left\{N_{i, p}\right\}_{i=1}^{n}$.

2. A control net in $d_{s}$-dimensional physical space ${ }^{3}$. This is given by a set of $n$ control points, $\left\{\mathbf{P}_{i}\right\}_{i=1}^{n}$, that lie in $\mathbb{R}^{d_{s}}$.

To define the B-spline basis in parameter space, we first define a knot vector, a set of non-decreasing coordinates in parameter space, $\Xi=\left\{\begin{array}{lll}\xi_{1} & \xi_{2} \ldots \xi_{n+p+1}\end{array}\right\}$. The B-spline basis functions of degree $p$ are then defined via the Cox-deBoor recursion formula as:

$$
\begin{gathered}
N_{i, 0}(\xi):=\left\{\begin{array}{l}
1 \text { if } \xi_{i} \leq \xi<\xi_{i+1} \\
0 \text { otherwise }
\end{array}\right. \\
N_{i, p}(\xi):=\frac{\xi-\xi_{i}}{\xi_{i+p}-\xi_{i}} N_{i, p-1}(\xi)+\frac{\xi_{i+p+1}-\xi}{\xi_{i+p+1}-\xi_{i+1}} N_{i+1, p-1}(\xi) \quad \forall \xi \in\left[\xi_{1}, \xi_{n+p+1}\right]
\end{gathered}
$$

\footnotetext{
${ }^{2}$ We note that CSG representations are also commonly encountered in CAD packages, but as we are specifically interested in the problem of surface-to-volume parameterization, we limit our discussion to BReps.

${ }^{3}$ Here, the subscript $r$ in $d_{r}$ stands for reference, and the subscript $s$ in $d_{s}$ stands for spatial.
} 
The desired B-spline curve is then defined as the sum of the B-spline control points multiplied with their respective basis functions, viz.:

$$
\mathbf{C}(\xi)=\sum_{i=1}^{n} N_{i, p}(\xi) \mathbf{P}_{i}
$$

As their name implies, NURBS are a generalization of B-splines, with the key difference being that each NURBS control point has an associated weight, and the NURBS basis is a rational basis. To define a NURBS curve, we require:

1. A set of $n$ B-spline basis functions $\left\{N_{i}\right\}_{i=1}^{n}$, in $\mathbb{R}^{d_{r}}$.

2. A set of $n$ control points, $\left\{\mathbf{P}_{i}\right\}_{i=1}^{n}$, in $\mathbb{R}^{d_{s}}$.

3. A set of $n$ control weights, $\left\{w_{i}\right\}_{i=1}^{n}$.

Physically, a NURBS curve is the projective mapping of a B-spline curve in $\mathbb{R}^{d_{s}+1}$ to $\mathbb{R}^{d_{s}}$. The control points of the B-spline curve in $\mathbb{R}^{d_{s}+1}$ are the projective or homogenous NURBS control points, $\widetilde{\mathbf{P}}_{i}$. The homogenous NURBS control points are found as:

$$
\begin{array}{r}
\left(\widetilde{\mathbf{P}}_{i}\right)_{j}=\left(\mathbf{P}_{i}\right)_{j} w_{i} \quad i \in\{1, \ldots, n\}, \quad j \in\left\{1, \ldots, d_{s}\right\} \\
\left(\widetilde{\mathbf{P}}_{i}\right)_{d_{s}+1}=w_{i}
\end{array}
$$

Numerically, a NURBS curve is defined as a linear combination of the product of the NURBS control points with their respective basis functions, viz.:

$$
\mathbf{C}(\xi)=\sum_{i=1}^{n} R_{i, p}(\xi) \mathbf{P}_{i}=\sum_{i=1}^{n} \frac{N_{i, p}(\xi) w_{i}}{\sum_{j=1}^{n} N_{j, p}(\xi) w_{j}} \mathbf{P}_{i}
$$

where the NURBS basis, $\left\{R_{i}\right\}_{i=1}^{n}$, is defined from the B-spline basis and control point weights via the relation:

$$
R_{i, p}(\xi)=\frac{N_{i, p}(\xi) w_{i}}{\sum_{j=1}^{n} N_{j, p}(\xi) w_{j}}
$$

Note that when all the control point weights are identically unity, the NURBS basis functions reduce to the B-spline basis functions, and the corresponding NURBS curve reduces to a B-spline curve. In this sense, the B-spline basis functions and B-spline curves are a restricted subset of the NURBS basis and NURBS curves, respectively. From an analysis point of view, the NURBS basis exhibits several beneficial properties, including pointwise non-negativity, partition of unity, linear independence and compact support. From a geometric modeling point of view, NURBS curves exhibit the beneficial properties of affine covariance and a strong convex hull.

Extending our discussion to NURBS surfaces is relatively straight forward, as the bivariate NURBS basis is simply the tensor product of two sets of univariate NURBS basis 
functions of degrees $p_{1}$ and $p_{2}$, respectively. That is, we define the bivariate $\left(d_{r}=2\right)$ NURBS basis functions as:

$$
R_{i j}(\boldsymbol{\xi})=\frac{N_{i, p_{1}}\left(\xi_{1}\right) N_{j, p_{2}}\left(\xi_{2}\right) w_{i j}}{\sum_{r=1}^{n} \sum_{s=1}^{m} N_{r, p_{1}}\left(\xi_{1}\right) N_{s, p_{2}}\left(\xi_{2}\right) w_{r s}}
$$

and we can then define a NURBS surface as:

$$
\boldsymbol{S}(\boldsymbol{\xi})=\sum_{i=1}^{n} \sum_{j=1}^{m} R_{i j}(\boldsymbol{\xi}) \mathbf{P}_{i j}=\sum_{i=1}^{n} \sum_{j=1}^{m} \frac{N_{i, p_{1}}\left(\xi_{1}\right) N_{j, p_{2}}\left(\xi_{2}\right) w_{i j}}{\sum_{r=1}^{n} \sum_{s=1}^{m} N_{r, p_{1}}\left(\xi_{1}\right) N_{s, p_{2}}\left(\xi_{2}\right) w_{r s}} \mathbf{P}_{i j}
$$

Figure 1a shows a representative bivariate NURBS basis function, and Figure 1b shows the corresponding control net and resulting NURBS surface.

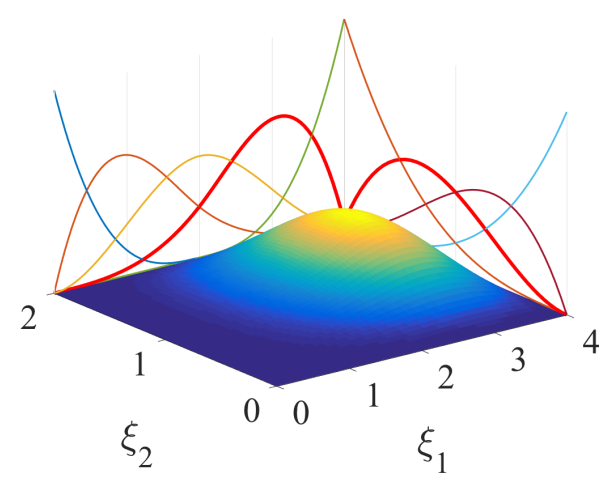

(a)

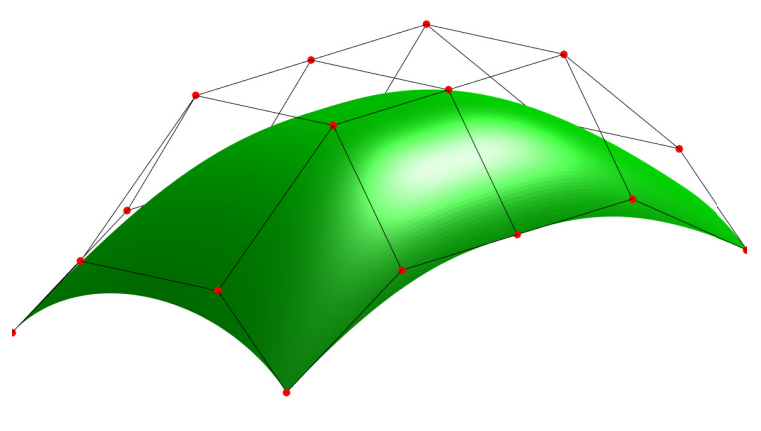

(b)

Figure 1: (a) The NURBS basis function $R_{4,3}$. (b) Corresponding NURBS control net and resultant NURBS surface.

\subsection{B-Reps}

As we have already alluded to, the surface-to-volume problem arises from the fact that in CAD packages, solid objects are visually represented as a collection of surfaces. These representations of solid objects by a collection of surfaces are called boundary representations, or B-Reps. Along with NURBS, B-Reps have long been the standard in CAD packages, due to their relative ease of implementation and flexibility in representing a wide array of complex geometries.

When constructing B-Reps, NURBS surfaces are the predominant geometric entities used for representing the bounding surfaces. The collection of NURBS surfaces comprising a B-Rep is known as a multi-patch NURBS surface. With multipatch NURBS surfaces, we are able to represent a wide array of geometries. Figure 2 shows some simple examples of multipatch geometries, with the individual NURBS patches shown in different colors. 
However, while many geometries can be represented using multi-patch NURBS surfaces, there are certain surfaces that cannot be represented in this way. This is predominantly due to the tensor product nature of the NURBS basis, which requires that any surface being represented by a NURBS surface be mappable to a rectangle in parametric space.

Traditionally, this limitation has been overcome by the introduction of trimming curves. Complex objects are first modeled using a collection of NURBS surfaces. In the locations where these surfaces intersect, the modeler introduces trimming curves to truncate the respective surfaces. The trimmed surfaces can then be connected along this trimming curve. We provide context for our discussion of B-Reps and trimming curves by considering the simple (yet commonly encountered) example of modeling the T-junctions of two cylinders, shown in Figure 3. First, the NURBS surfaces defining the surfaces of the cylinder are created (Figure 3a). Next, a trimming curve is created along the intersection of the two cylinders, and the overlapping segments are removed. Figures $3 \mathrm{~b}$ and c show this process, with the trimmed sections highlighted in red, and the resulting surface is shown in Figure $3 \mathrm{~d}$. Next, the ends of the cylinders, are capped with trimmed NURBS surfaces (Figure 3e). The final geometry is then defined by the volume bounded by the union of the trimmed surfaces, shown in Figure 3f.

While NURBS and B-Reps are undoubtably powerful tools for CAD, as evidenced by their widespread use, there are some notable disadvantages to these representations. First, whereas a NURBS parameterization is explicit, the introduction of trimming curves causes the underlying parameterization to be implicit, which is undesirable from a numerical analysis standpoint. Additionally, multi-patch geometries often have gaps and overlaps, and we therefore say they are not watertight. While these discontinuities in the geometry may not be large, and thus not readily apparent in CAD, they pose serious problems in the analysis framework. For instance, gaps and overlaps in geometry result in crack-tip stress concentrations in structural analysis and leakage in fluid flow simulations. Finally, it is impossible to perform local refinement of a NURBS surface. Because of the tensor product nature of the NURBS basis, any refinement must propagate across an entire NURBS patch. It is these shortcomings that motivated the advent of T-splines as an alternative to NURBS based B-Reps in CAD packages [32].

\section{$2.3 \quad$ T-splines}

T-splines are a generalization of NURBS that allow for T-junctions in the parameterization $[4,32]$. To define a NURBS basis, one must simply define the knot vectors in each parametric direction that define the basis. Because the knot span is then defined as the tensor product of these knot vectors, NURBS are inherently a structured basis. T-splines on the other hand allow for more geometric flexibility by defining a set of local knot vectors for each basis function. Each local set is made up of $d_{r}$ knot vectors, one in each parametric direction, with each knot vector defining a single basis function, $N_{\alpha}^{l}$. Once we have defined the local knot vectors and their corresponding basis functions, we can then define a single $d_{r}$-variate basis function for each control point, $\mathbf{P}_{\alpha}$, viz:

$$
B_{\alpha}(\boldsymbol{\xi})=\prod_{l=1}^{d_{r}} N_{\alpha}^{l}\left(\xi^{l}\right)
$$




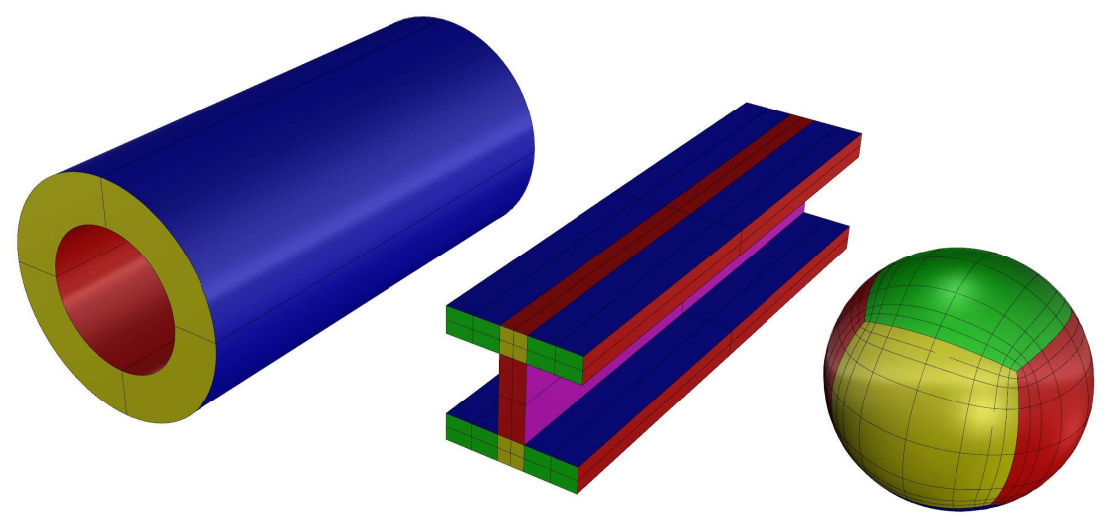

Figure 2: Examples of multipatch NURBS surfaces. Each NURBS patch is shown in a different color.

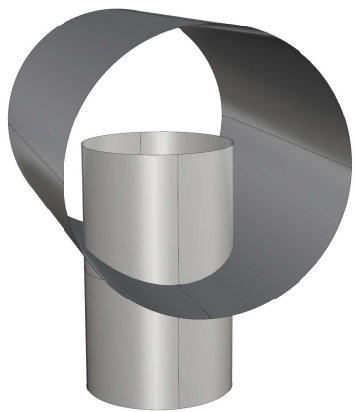

(a)

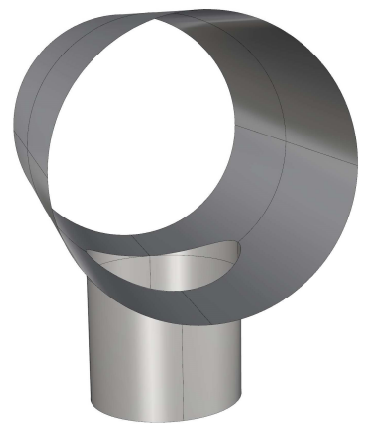

(d)

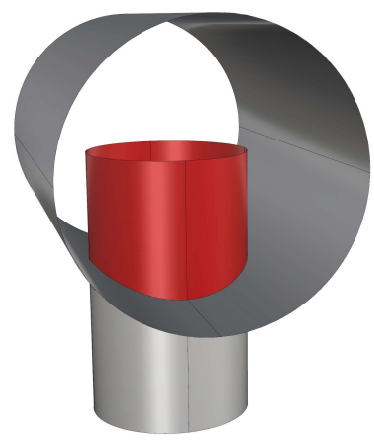

(b)

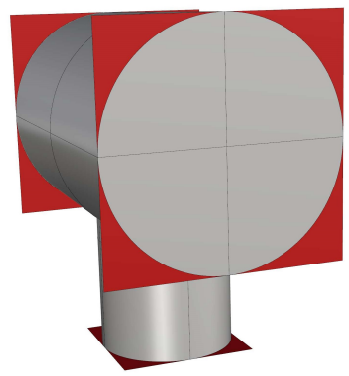

(e)

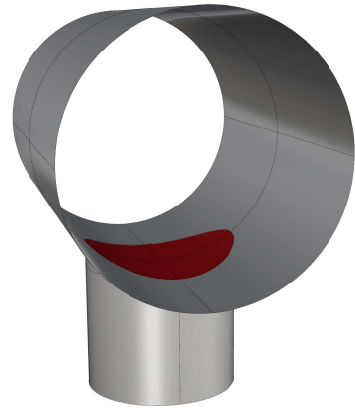

(c)

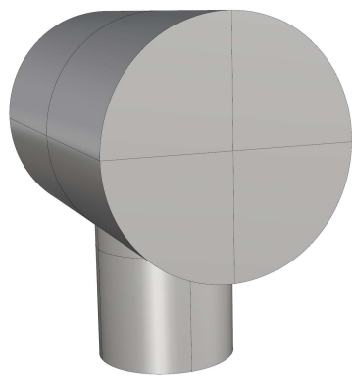

(f)

Figure 3: Creating a junctions of two cylinders using trimming curves. 
Consider, for example, the point $\mathbf{P}_{\{3,4\}}$ with local knot vectors $\Xi_{1}=\left[\begin{array}{lllll}1 & 2 & 3 & 4 & 5\end{array}\right]$ and $\Xi_{2}=\left[\begin{array}{lllll}2 & 3 & 4 & 5 & 6\end{array}\right]$ shown in Figure 4a. We can define the cubic basis functions $N_{3}^{1}$ and $N_{4}^{2}$ over their respective local knot vectors (Figure $4 \mathrm{~b}$ ). Then, the bivariate basis function, $B_{\{3,4\}}$, is defined as the tensor product of the two univariate basis functions, $B_{\{3,4\}}=N_{3}^{1} \otimes N_{4}^{2}$ (Figure 4c).

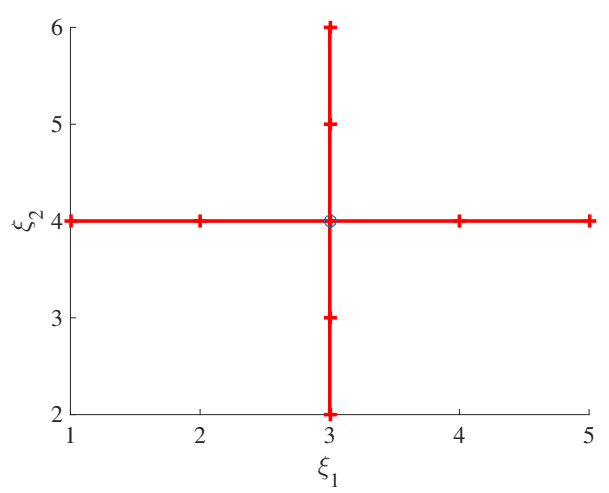

(a)

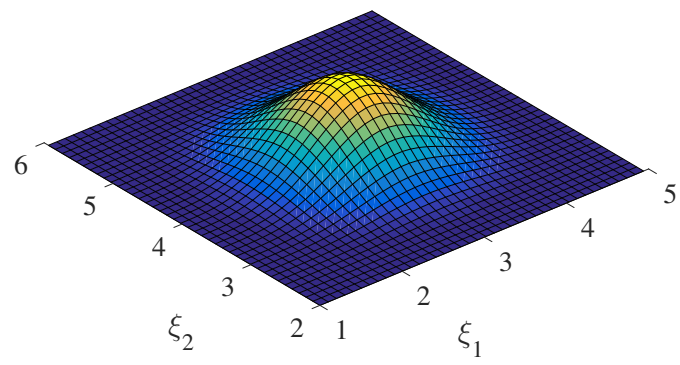

(c)

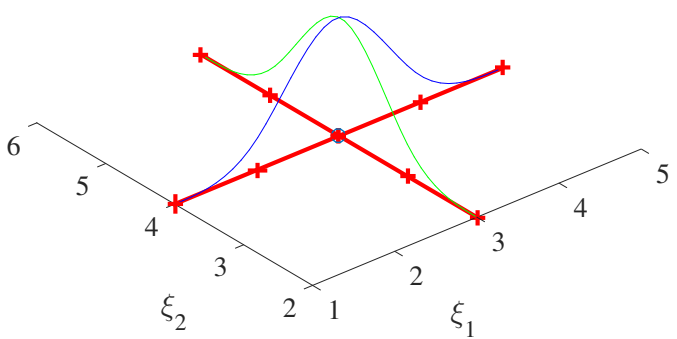

(b)

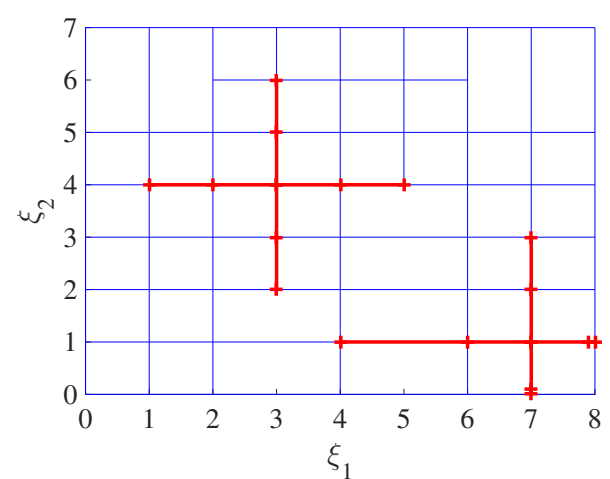

(d)

Figure 4: (a) The local knot vectors. (b) Local univariate basis functions defined over the knot vectors. (c) The resulting local bivariate basis function. (d) A T-mesh with the representative local knot vectors shown in red.

Thus, much like with NURBS surfaces, each control point $\mathbf{P}_{\alpha}$ in physical space will have a corresponding basis function defined in parametric space, $B_{\alpha}$. Then, to define a T-spline surface, we need a set of T-spline control points, as well as a way to relate these control points to each other. This is done via a T-mesh, which defines the topology and parameterization for the corresponding T-spline surface. A representative T-mesh for a cubic T-spline is shown in Figure 4d. Note that it is at this point, that the theory for T-splines diverges slightly. For odd polynomial degree T-splines, each vertex in the T-mesh defines an anchor, $s_{\alpha}$, and there is a direct mapping from every anchor in the T-mesh to every control point, $\mathbf{P}_{\alpha}$ in physical space. For even polynomial degree, the anchors are instead defined at the 
center of each face in the T-mesh. However, bi-cubic T-splines have come to be the de-facto standard in the CAD community, so we limit our discussion of T-splines to this case. Also shown in Figure 4 are representative local knot vectors for the anchors $s_{\{3,4\}}$, and $s_{\{7,1\}}$, where the hash marks indicate the location of a knot. Observe that for a cubic T-spline, the local knot vectors are formed by extending a line in each parametric direction, until the line intersects with two orthogonal T-mesh lines in each direction. For the anchor $s_{\{3,4\}}$ the local knot vectors are $\Xi_{1}=\left[\begin{array}{lllll}1 & 2 & 3 & 4 & 5\end{array}\right]$ and $\Xi_{2}=\left[\begin{array}{lllll}2 & 3 & 4 & 5 & 6\end{array}\right]$. In the case of the anchor $s_{\{7,1\}}$, the local knot vectors are $\Xi_{1}=\left[\begin{array}{lllll}4 & 6 & 7 & 8 & 8\end{array}\right]$ and $\Xi_{2}=\left[\begin{array}{lllll}0 & 0 & 1 & 2 & 3\end{array}\right]$, where we have repeated knots because of the intersection with the T-mesh boundary.

Finally, as with NURBS, each T-spline control point has an associated control weight. Thus, for each anchor in the T-mesh, we define a set of T-spline blending functions:

$$
R_{\alpha}(\xi)=\frac{w_{\alpha} B_{\alpha}(\xi)}{\sum_{\beta \in A} w_{\beta} B_{\beta}(\xi)}
$$

With these blending functions defined, we can then define our T-spline in physical space as:

$$
S(\boldsymbol{\xi})=\sum_{\alpha=A} \mathbf{P}_{\alpha} R_{\alpha}(\boldsymbol{\xi})
$$

Figure 5b shows a T-spline surface, and Figure 5a shows the corresponding T-mesh. Note the direct mapping of anchors in the T-mesh to control points of the T-spline. Finally, it is worth noting that NURBS are simply a restricted subset of T-splines that do not permit T-junctions.

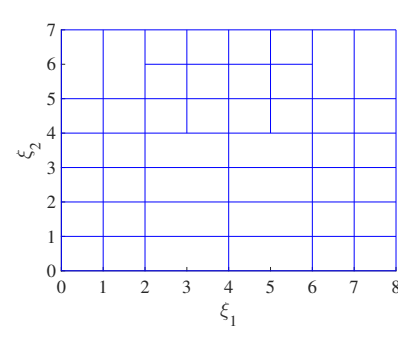

(a)

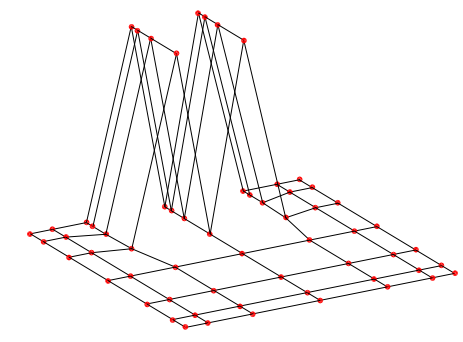

(b)

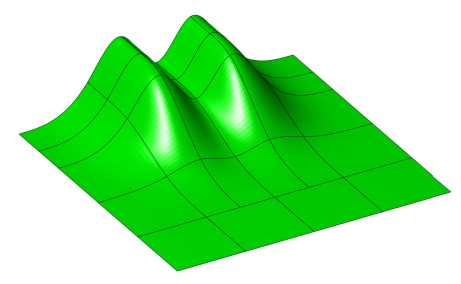

(c)

Figure 5: (a) The T-mesh in parametric space. (b) The T-spline control net. (c) The corresponding T-spline surface.

A substantial amount of work has been done in recent years on the applications of Tsplines, both in the context of design and analysis. In this paper, we use T-splines only from the design point of view, as mathematical representations of surfaces from CAD. In this context, T-spline surfaces exhibit many of the nice properties of NURBS surfaces such as affine covariance and a strong convex hull. However, is it worth mentioning that using Tsplines as basis for isogeometric analysis is an area of active research. As they are presented here, T-spline blending functions are not necessarily linearly independent. However, it has been shown that we can define a restricted subset of T-splines, called analysis-suitable $\boldsymbol{T}$-splines, which are guaranteed to exhibit linear independence, point-wise non-negativity 
and partition of unity [20,31]. Finally, as we have discussed at length in our previous paper, much work has been done to solve the surface-to-volume parameterization problem using T-splines. While there have been many proposed methods for creating trivariate T-spline discretizations for the use in isogeometric analysis, this is still largely an unsolved problem. Because of this, we limit our treatment of T-splines in this paper to the bi-variate case, and focus on their use as purely a design technology for representing surfaces in CAD.

\section{Meshing Geometric Primitives}

We continue this paper by proceeding forward to the construction of analysis-suitable, high-order, geometrically exact volumetric meshes. We begin our discussion with the Bézier geometric primitives that comprise these meshes. These are hexahedra, tetrahedra, wedges, and pyramids. Note that mesh generation can be done using other geometric primitives (arbitrary polyhedra), but the four primitives discussed in this paper are the most commonly used polyhedra in mesh generation, and are sufficient for our meshing purposes. Additionally, we provide a gallery of Bézier geometric primitives for the cubic case.

\subsection{The Bernstein-Bézier Form}

Generally speaking, to define an arbitrary Bézier element $\Omega$, we need three items:

1. Bernstein basis functions defined over a reference element in parametric space $\left\{B_{i}(\boldsymbol{\xi})\right\}_{i \in I}$.

2. A control net defining the element in physical space $\left\{\mathbf{P}_{i}^{b}\right\}_{i \in I}$, and

3. Corresponding weights for each control point in the control net $\left\{w_{i}\right\}_{i \in I}$.

Here, $I$ is an index set defining the ordering of the control points on the reference geometry. The Bézier element is then defined using a linear combination of the product of rational Bernstein-Bézier basis functions with their respective control points, viz:

$$
\vec{x}(\boldsymbol{\xi})=\sum_{i \in I} R_{i}(\boldsymbol{\xi}) \mathbf{P}_{i}^{b}
$$

where $\left\{R_{i}(\boldsymbol{\xi})\right\}_{i \in I}$ are the rational Bernstein-Bézier basis functions, defined as:

$$
R_{i}(\boldsymbol{\xi})=\frac{B_{i}(\boldsymbol{\xi}) w_{i}}{\sum_{j \in I} B_{j}(\boldsymbol{\xi}) w_{j}}
$$

The Bernstein polynomials, and the resulting rational Bernstein-Bézier basis functions, exhibit several nice properties that make them a suitable as a set of basis functions for finite element analysis. These include partition of unity, point-wise non-negativity, linear independence and compact support. From our general definition of a Bézier element in Equation (13), together with the properties of the Bernstein polynomials, it can be shown that any Bézier element will exhibit affine covariance, and a strong convex hull. Most importantly, note that in order to define an arbitrary Bézier element, we need only to be able to define the Bernstein polynomials over a corresponding parametric reference geometry. 
With this in mind, the rest of this section will provide more concrete examples for the geometries of interest in this paper. We start by considering Bézier Curves, Quadrilaterals and Triangles, before considering the four geometric primitives used in meshing. This approach illuminates an important property of all Bézier elements: the boundary of a Bézier element is simply another Bézier element. That is, the faces of Bézier hexahedra are simply Bézier quadrilaterals, and the faces of Bézier tetrahedra are simply Bézier triangles. Likewise, the edges of Bézier quadrilaterals and triangles are simply Bézier curves.

At this juncture, it is useful to make a few remarks regarding terminology. We use the clarifier "Bernstein" when referring to the Bernstein polynomial basis and the clarifier "Bézier" when referring to an element in physical space or the control net defining said element. We use the clarifier "Bernstein-Bézier" when referring to the rational basis or the parametrization of the element as these involve both the Bernstein basis and the Bézier control net.

\subsection{Curves}

Bézier curves are the simplest of all Bézier elements considered in this paper. Let the reference curve in parametric space be defined as the unit line:

$$
\hat{\Omega}^{\text {curve }}=(0,1)
$$

Then a Bézier curve of polynomial degree $p$ is simply defined using the mapping:

$$
\vec{x}^{\text {curve }}(\xi)=\sum_{i=0}^{p} R_{i, p}(\xi) \mathbf{P}_{i}^{b} \quad \forall \xi \in \hat{\Omega}^{\text {curve }}
$$

where $\left\{R_{i, p}\right\}_{i=0}^{p}$ are the univariate rational Bernstein-Bézier basis functions, defined as:

$$
R_{i, p}(\xi)=\frac{B_{i, p}(\xi) w_{i}}{\sum_{j=0}^{p} B_{j, p}(\xi) w_{j}}
$$

and $\left\{B_{i, p}\right\}_{i=0}^{p}$ are the univariate Bernstein polynomials of degree $p$, defined as:

$$
B_{i, p}(\xi)=\left(\begin{array}{c}
p \\
i
\end{array}\right) \xi^{i}(1-\xi)^{i-p}
$$

Note that the Bernstein polynomials form a restricted subset of the B-spline basis that arise when evaluating the B-spline basis functions over the knot vector: $\Xi=[\underbrace{0 \ldots 0}_{\mathrm{p}+1 \text { times } \mathrm{p}+1 \text { times }} \underbrace{1 \ldots 1}]$. Also, for notational simplicity, for the remainder of this paper we choose to drop the subscript $p$ where the polynomial degree can be inferred from context.

\subsection{Quadrilaterals}

Bézier quadrilaterals are defined from tensor product Bernstein polynomials. Thus, we first define the reference quadrilateral as: 


$$
\hat{\Omega}^{\text {quad }}=(0,1)^{2}
$$

and then define a Bézier quadrilateral of degree $\left(p_{1}, p_{2}\right)$ using the mapping:

$$
\vec{x}^{\text {quad }}(\boldsymbol{\xi})=\sum_{i=0}^{p_{1}} \sum_{j=0}^{p_{2}} R_{i j}^{q u a d}(\boldsymbol{\xi}) \mathbf{P}_{i j}^{b} \quad \forall \boldsymbol{\xi} \in \hat{\Omega}^{\text {quad }}
$$

where the bivariate rational Bernstein-Bézier basis is defined as:

$$
R_{i j}^{q u a d}(\boldsymbol{\xi})=\frac{B_{i, p_{1}}\left(\xi_{1}\right) B_{j, p_{2}}\left(\xi_{2}\right) w_{i j}}{\sum_{r=0}^{p_{1}} \sum_{s=0}^{p_{2}} B_{r, p_{1}}\left(\xi_{1}\right) B_{s, p_{2}}\left(\xi_{2}\right) w_{r s}}
$$

Figure 6a shows the bicubic Bernstein-Bézier control net in parametric space, and Figure 6b shows a corresponding bicubic Bézier quadrilateral in physical space.

\subsection{Triangles}

We first define the unit reference triangle in parameter space as:

$$
\hat{\Omega}^{t r i}=\left\{\left(\xi_{1}, \xi_{2}\right) \in \mathbb{R}^{2}: \xi_{1}, \xi_{2} \geq 0, \xi_{1}+\xi_{2} \leq 1\right\}
$$

Then a Bézier triangle of degree $p$ is defined using the mapping:

$$
\vec{x}^{t r i}(\boldsymbol{\xi})=\sum_{i+j+k=p} R_{i j k}^{t r i}(\boldsymbol{\xi}) \mathbf{P}_{i j k}^{b} \quad \forall \boldsymbol{\xi} \in \hat{\Omega}^{t r i}
$$

where we define the triangular rational Bernstein-Bézier basis functions in terms of the barycentric coordinates as:

$$
\begin{array}{r}
R_{i j k}^{t r i}(\boldsymbol{\xi})=\frac{B_{i j k}^{t r i}(\boldsymbol{\xi}) w_{i j k}}{\sum_{r+s+t=p} B_{r s t}^{t r i}(\boldsymbol{\xi}) w_{r s t}} \\
i+j+k=p \\
i, j, k \in[0, p]
\end{array}
$$

and the Bernstein polynomials of degree $p$ over a triangle as:

$$
B_{i j k}^{t r i}(\boldsymbol{\xi})=\frac{p !}{i ! j ! k !} \xi_{1}^{i} \xi_{2}^{j}\left(1-\xi_{1}-\xi_{2}\right)^{k}
$$

Figures $6 \mathrm{c}$ and $6 \mathrm{~d}$ show the control net for a cubic Bézier triangle in parametric space and physical space. 


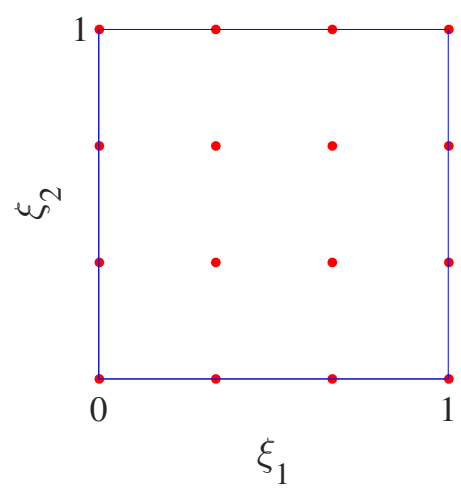

(a)

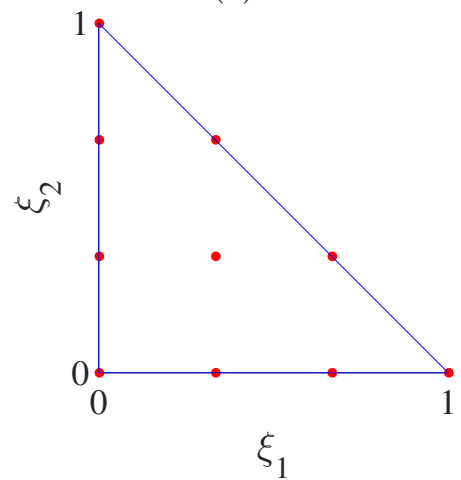

(c)

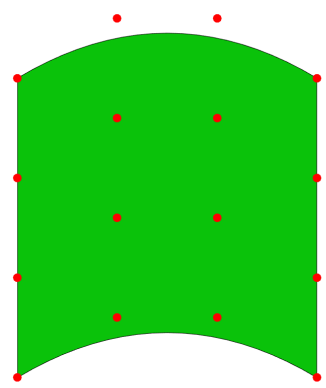

(b)

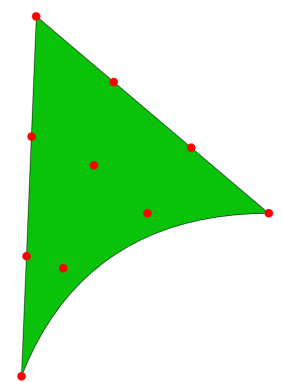

(d)

Figure 6: Bi-variate Bézier element gallery. (a) The bicubic quadrilateral control net in parametric space. (b) A bicubic Bézier quadrilateral in physical space. (c) The cubic triangular control net in parametric space. (d) A cubic Bézier triangle in physical space. 


\subsection{Hexahedra}

Like Bézier quadrilaterals, Bézier hexahedra are defined from tensor product Bernstein polynomials. Thus, we first define the reference hexahedron as:

$$
\hat{\Omega}^{h e x}=(0,1)^{3}
$$

and then define a Bézier hexahedron of degree $\left(p_{1}, p_{2}, p_{3}\right)$ using the mapping:

$$
\vec{x}^{h e x}(\boldsymbol{\xi})=\sum_{i=0}^{p_{1}} \sum_{j=0}^{p_{2}} \sum_{k=0}^{p_{3}} R_{i j k}^{h e x}(\boldsymbol{\xi}) \mathbf{P}_{i j k}^{b} \quad \forall \boldsymbol{\xi} \in \hat{\Omega}^{h e x}
$$

where $\left\{\mathbf{P}_{i j k}^{b}\right\}_{i, j, k}$ denotes the control net in physical space, and $\left\{R_{i j k}^{h e x}(\boldsymbol{\xi})\right\}_{i, j, k}$ are the trivariate rational Bernstein-Bézier basis functions, defined as:

$$
R_{i j k}^{h e x}(\boldsymbol{\xi})=\frac{B_{i, p_{1}}\left(\xi_{1}\right) B_{j, p_{2}}\left(\xi_{2}\right) B_{k, p_{3}}\left(\xi_{3}\right) w_{i j k}}{\sum_{r=0}^{p_{1}} \sum_{s=0}^{p_{2}} \sum_{t=0}^{p_{3}} B_{r, p_{1}}\left(\xi_{1}\right) B_{s, p_{2}}\left(\xi_{2}\right) B_{t, p_{3}}\left(\xi_{3}\right) w_{r s t}}
$$

Figure 7a shows the control net for the tricubic Bézier hexahedron in parametric space, and Figure $7 \mathrm{~b}$ shows a corresponding tricubic Bézier hexahedron in physical space.

\subsection{Tetrahedra}

We first define the reference tetrahedron as the unit trirectangular tetrahedron:

$$
\hat{\Omega}_{t e t}=\left\{\left(\xi_{1}, \xi_{2}, \xi_{3}\right) \in \mathbb{R}^{3}: \xi_{1}, \xi_{2}, \xi_{3} \geq 0, \xi_{1}+\xi_{2}+\xi_{3} \leq 1\right\}
$$

A Bézier tetrahedron of degree $p$ is then defined using the mapping:

$$
\vec{x}^{t e t}(\boldsymbol{\xi})=\sum_{i+j+k+l=p} R_{i j k l}^{t e t}(\boldsymbol{\xi}) \mathbf{P}_{i j k l}^{b} \quad \forall \boldsymbol{\xi} \in \hat{\Omega}^{t e t}
$$

where we define the rational Bernstein-Bézier basis functions over a tetrahedron as:

$$
\begin{array}{r}
R_{i j k l}^{t e t}(\boldsymbol{\xi})=\frac{B_{i j k l}^{t e t}(\boldsymbol{\xi}) w_{i j k l}}{\sum_{r+s+t+u=p} B_{r s t u}^{t e t}(\boldsymbol{\xi}) w_{r s t u}} \\
i+j+k+l=p \\
i, j, k, l \in[0, p]
\end{array}
$$

and the Bernstein polynomials of degree $p$ over a tetrahedron are defined as:

$$
B_{i j k l}^{\text {tet }}(\boldsymbol{\xi})=\frac{p !}{i ! j ! k ! l !} \xi_{1}^{i} \xi_{2}^{j} \xi_{3}^{k}\left(1-\xi_{1}-\xi_{2}-\xi_{3}\right)^{l}
$$

Figure 7c shows the control net for a Bézier tetrahedron in parametric space, and Figure $7 \mathrm{~d}$ shows the corresponding Bézier tetrahedron in physical space. 


\subsection{Wedges}

A Bézier wedge is defined from the tensor product of Bernstein polynomials over a triangle with the univariate Bernstein polynomials. Thus, we define the reference wedge in parameter space as:

$$
\hat{\Omega}^{\text {wedge }}=\left\{\left(\xi_{1}, \xi_{2}, \xi_{3}\right) \in \mathbb{R}^{3}: \xi_{1}, \xi_{2}, \xi_{3} \geq 0, \xi_{1}+\xi_{2} \leq 1, \xi_{3} \leq 1\right\}
$$

and the Bézier wedge of degree $p$ in physical space using the mapping:

$$
\vec{x}^{\text {wedge }}(\boldsymbol{\xi})=\sum_{l=0}^{p} \sum_{i+j+k=p} R_{i j k l}^{\text {wedge }}(\boldsymbol{\xi}) \mathbf{P}_{i j k l}^{b} \quad \forall \boldsymbol{\xi} \in \hat{\Omega}^{\text {wedge }}
$$

where the basis functions $\left\{R_{i j k l}^{\text {wedge }}(\boldsymbol{\xi})\right\}_{i, j, k, l}$ are defined from the tensor product of triangular Bernstein basis functions and univariate Bernstein basis functions, viz.:

$$
R_{i j k l}^{\text {wedge }}(\boldsymbol{\xi})=\frac{B_{i j k}^{t r i}\left(\xi_{1}, \xi_{2}\right) B_{l}\left(\xi_{3}\right) w_{i j k} w_{l}}{\sum_{r+s+t=p} \sum_{u=0}^{p} B_{r s t}^{t r i}\left(\xi_{1}, \xi_{2}\right) B_{u}\left(\xi_{3}\right) w_{r s t} w_{u}}
$$

Figure 7e shows the control net for the cubic Bézier wedge in parametric space, and Figure $7 \mathrm{f}$ shows a corresponding cubic Bézier wedge in physical space.

\subsection{Pyramids}

Bézier pyramids take more care to define than the other primitives considered in this paper. First, let us define the unit reference pyramid as:

$$
\hat{\Omega}^{\text {pyr }}=\left\{\left(\xi_{1}, \xi_{2}, \xi_{3}\right) \in \mathbb{R}^{3}: \xi_{1}, \xi_{2}, \xi_{3} \geq 0, \xi_{1}+\xi_{3} \leq 1, \xi_{2}+\xi_{3} \leq 1, \xi_{3} \leq 1\right\}
$$

However, unlike the other primitives considered in the paper, we do not have a notion of Bernstein polynomials defined over $\hat{\Omega}^{p y r}$. Rather, Chan et. al. [7] recently showed that the Bernstein basis of degree $p$ for a pyramid can first be defined over the unit cube as:

$$
B_{i j k}(\boldsymbol{a})=B_{i, p-k}(a) B_{j, p-k}(b) B_{k, p}(c) \quad \forall \boldsymbol{a}=(a, b, c) \in \hat{\Omega}^{h e x}
$$

We can then map the basis to the reference pyramid via the Duffy transform. That is, the coordinates $(a, b, c)$ are found from $\left(\xi_{1}, \xi_{2}, \xi_{3}\right)$ as:

$$
\begin{array}{r}
a=\frac{\xi_{1}}{1-\xi_{3}} \\
b=\frac{\xi_{2}}{1-\xi_{3}} \\
c=\xi_{3}
\end{array}
$$

and we can then define the rational Bernstein-Bézier basis over the pyramid as: 


$$
R_{i j k}^{p y r}(\boldsymbol{\xi})=\frac{B_{i, p-k}(a(\boldsymbol{\xi})) B_{j, p-k}(b(\boldsymbol{\xi})) B_{k, p}(c(\boldsymbol{\xi})) w_{i j k}}{\sum_{t=0}^{p} \sum_{r=0}^{p-t} \sum_{s=0}^{p-t} B_{r, p-t}(a(\boldsymbol{\xi})) B_{s, p-t}(b(\boldsymbol{\xi})) B_{t, p}(c(\boldsymbol{\xi})) w_{r s t}}
$$

We can finally define a Bernstein-Bézier pyramid of degree $p$ using the mapping:

$$
\vec{x}^{p y r}(\boldsymbol{\xi})=\sum_{k=0}^{p} \sum_{j=0}^{p-k} \sum_{i=0}^{p-k} R_{i j k}^{p y r}(\boldsymbol{\xi}) \mathbf{P}_{i j k}^{b} \quad \forall \boldsymbol{\xi} \in \hat{\Omega}^{p y r}
$$

The Duffy transform can also be employed to define the Bernstein basis for a triangle by collapsing the coordinate system for a quadrilateral. Under the Duffy transform, the polynomial basis over the unit cube is transformed to a rational basis over the reference pyramid. Nevertheless, it can be shown that this rational basis is complete in that it contains all polynomials of degree $p$. Moreover, it can be shown that over each triangular face of the pyramid, the trace of the rational basis is spanned by the triangular Bernstein basis of degree $p$, and over the rectangular face of the pyramid, the trace is spanned by the tensorproduct Bernstein basis of degree $(p, p)$. It can also be demonstrated that the Bernstein basis over a pyramid maintains all the beneficial properties of the Bernstein polynomials, such as partition of unity, point-wise non-negativity, and linear independence.

A cubic Bézier pyramid is defined by a 30-node control net. Figure $7 \mathrm{~g}$ shows the control net for the cubic Bézier pyramid in parametric space, and Figure $7 \mathrm{~h}$ shows a corresponding cubic Bézier pyramid in physical space. One very important property of the Bézier pyramid is that it serves as a transition element between Bézier hexahedra and Bézier tetrahedra.

\section{Bézier Extraction and Bézier Projection}

Thus far, we have presented NURBS and T-spline surfaces as methods for representing objects in $\mathrm{CAD}$, and we have presented various Bézier primitives to be used to create analysis suitable meshes. However, it remains to establish a relationship between these global NURBS and T-spline representations, and the local Bézier elements. Specifically, we would like to establish a relationship between NURBS curves and Bézier curves, and a relationship between NURBS or T-spline surfaces and Bézier surfaces. Note that because the faces of Bézier hexahedra, tetrahedra, wedges and pyramids are themselves Bézier quadrilaterals or triangles, this will then give us a relationship between CAD surfaces (NURBS/T-splines) and the faces of the Bézier meshing primitives presented above. The motivation for establishing this relationship will become clear in Section 6.3, where we use face swapping on Bézier elements to reconstruct the surface mesh to match the CAD geometry. For now, we introduce two tools necessary to do this task: Bézier extraction and Bézier projection.

In this section, and throughout the rest of this paper, it will be useful to introduce some additional notation. When working with sets of points, we often find it useful to arrange the members of the set into a matrix. We denote this matrix representation by dropping the index on the variable. That is, for a set of Bézier points, we write the matrix representation as: 


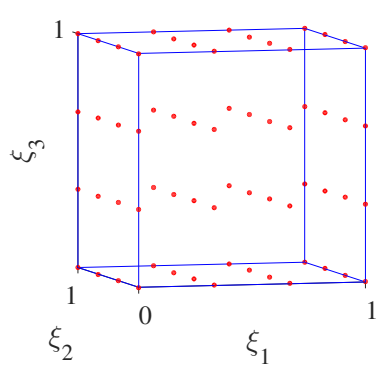

(a)

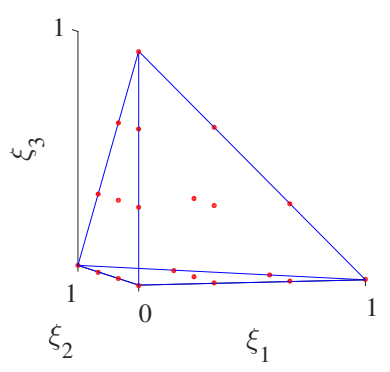

(c)

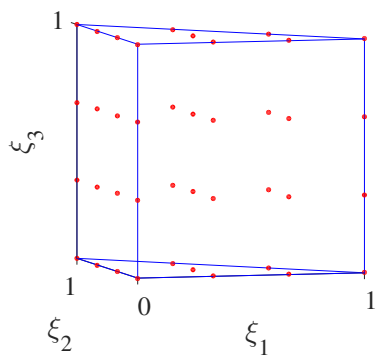

(e)

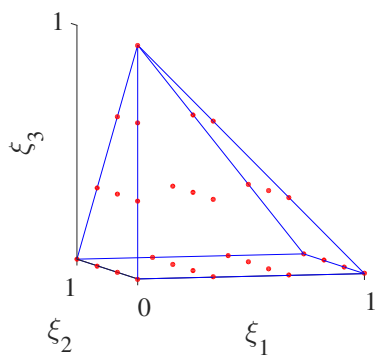

$(\mathrm{g})$

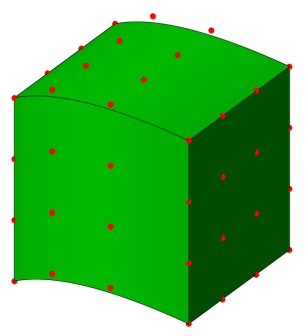

(b)

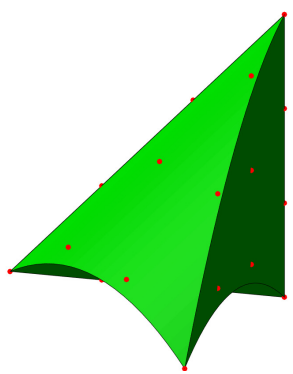

(d)

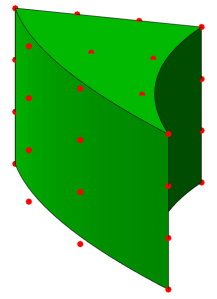

(f)

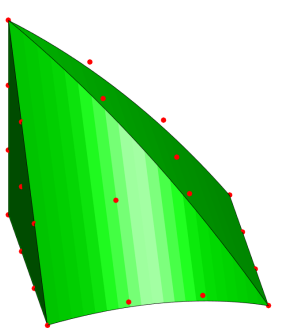

(h)

Figure 7: Meshing geometric primitives. (a-b) Tricubic Bézier hexahedra in parametric and physical space. (c-d) Cubic Bézier tetrahedra in parametric and physical space.(e-f) Cubic Bézier wedges in parametric and physical space.(g-h) Cubic Bézier pyramids in parametric and physical space. 


$$
\mathbf{P}^{b}=\left[\begin{array}{c}
\left(\mathbf{P}_{1}^{b}\right)^{T} \\
\left(\mathbf{P}_{2}^{b}\right)^{T} \\
\cdot \\
\cdot \\
\left(\mathbf{P}_{n}^{b}\right)^{T}
\end{array}\right]=\left[\begin{array}{c}
\left(\mathbf{P}_{1}^{b}\right)_{1}\left(\mathbf{P}_{1}^{b}\right)_{2} \ldots\left(\mathbf{P}_{1}^{b}\right)_{d_{s}} \\
\left(\mathbf{P}_{1}^{b}\right)_{2}\left(\mathbf{P}_{2}^{b}\right)_{2} \ldots\left(\mathbf{P}_{2}^{b}\right)_{d_{s}} \\
\cdot \\
\cdot \\
\left(\mathbf{P}_{n}^{b}\right)_{1}\left(\mathbf{P}_{n}^{b}\right)_{2} \ldots\left(\mathbf{P}_{n}^{b}\right)_{d_{s}}
\end{array}\right]
$$

\subsection{Bézier Extraction}

Bézier extraction was introduced by Borden et al. [6] as a means to express the relationship between the B-spline basis and the Bernstein basis via an element-wise extraction operator, $\mathbf{C}^{e}$. Consider the set of univariate B-spline basis functions $\left\{N_{i}\right\}_{i=1}^{n}$, and corresponding control points $\left\{\mathbf{P}_{i}\right\}_{i=1}^{n}$. Over any given knot interval $\xi \in\left[\xi_{m}, \xi_{m+1}\right)$, there exist a set of $p+1$ non-zero basis functions:

$$
\left\{N_{i}^{e}\right\}_{i=0}^{p}:=\left\{N_{i} \mid N_{i}(\xi)>0 \forall \xi \in\left[\xi_{m}, \xi_{m+1}\right)\right\}
$$

We can then define these local B-spline basis functions $\left\{N_{i}^{e}\right\}_{i=0}^{p}$ in terms of the Bernstein basis via the element extraction operator:

$$
N_{i}^{e}=\sum_{j} \mathbf{C}_{i j}^{e} B_{j}
$$

Now let us also denote the set of $p+1$ local control points that correspond to to the element B-spline basis functions as $\left\{\mathbf{P}_{i}^{e}\right\}_{i=0}^{p}$. We can then define the relationship between the element B-spline control points and the Bézier control points for the current element, $\left\{\mathbf{P}_{i}^{b, e}\right\}_{i=0}^{p}$ by the equation:

$$
\mathbf{P}^{b, e}=\left(\mathbf{C}^{e}\right)^{T} \mathbf{P}^{e}
$$

When performing Bézier extraction on NURBS curves, the process is the same, but we

perform extraction on the homogenous element NURBS control points $\left\{\widetilde{\mathbf{P}}_{i}^{e}\right\}_{i=0}^{p}$ to arrive at the homogenous Bézier control points $\left\{\widetilde{\mathbf{P}}_{i}^{b, e}\right\}_{i=0}^{p}$, viz:

$$
\widetilde{\mathbf{P}}^{b, e}=\left(\mathbf{C}^{e}\right)^{T} \widetilde{\mathbf{P}}^{e}
$$

where we can find the homogenous NURBS control points via Equation (56). Finally, the Bézier control points $\left\{\mathbf{P}_{i}^{b, e}\right\}_{0=1}^{p}$ and weights $\left\{w_{i}^{b, e}\right\}_{i=0}^{p}$, are recovered by :

$$
\begin{array}{r}
\left(\mathbf{P}_{i}^{b, e}\right)_{j}=\frac{\left(\widetilde{\mathbf{P}}_{i}^{b, e}\right)_{j}}{w_{i}^{b, e}} \quad i \in\{0, \ldots, p\}, j \in\left\{1, \ldots, d_{s}\right\} \\
w_{i}^{b}=\left(\widetilde{\mathbf{P}}_{i}^{b, e}\right)_{d_{s}+1}
\end{array}
$$

A nice property of Bézier extraction is that it extends directly to the multivariate case from the univariate case. In fact, in order to perform Bézier extraction of NURBS or Tspline surfaces, Equation (44) still holds, and we must simply define the appropriate element 
extraction operators. This is powerful as it allows any NURBS or T-spline geometry to be defined by a collection of Bézier elements, provided an extraction operator can be computed. Figure 8 shows the result of performing Bézier extraction on the T-spline surface from Figure 5. Note that the T-junctions remain in the Bézier extracted form of the T-spline surface. Interested readers are directed to Borden et al. [6] for details on computing extraction operators for NURBS curves and surfaces, and Scott et at. [30] for details on computing extraction operators for T-splines.

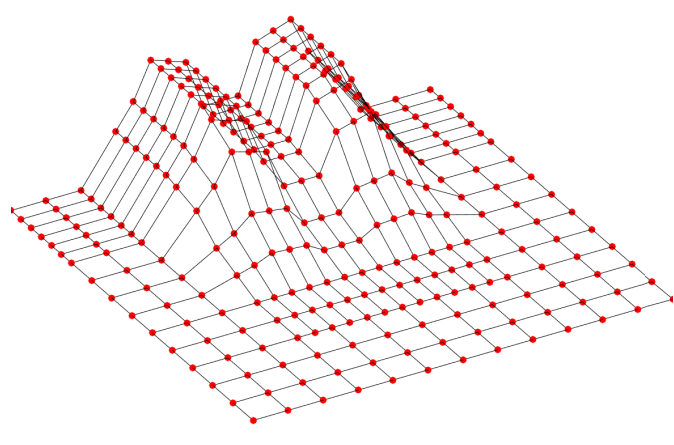

(a)

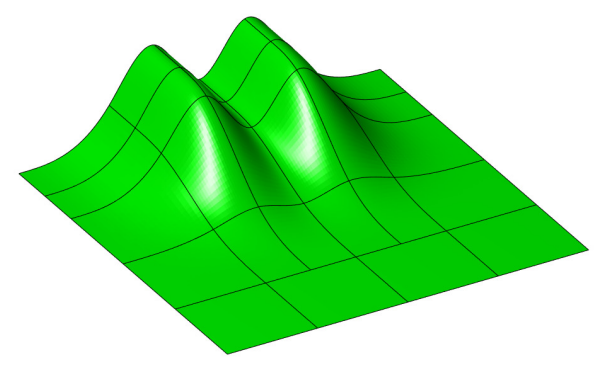

(b)

Figure 8: Bézier extraction on a T-spline surface. (a) The control net for the extracted Bézier elements. (b) The Bézier elements.

\subsection{Bézier Projection}

As stated earlier, we wish to define some relation between a global NURBS or T-spline surface, and triangular and quadrilateral faces of the Bézier elements that lie on the global boundary of the mesh. Bézier projection, proposed by Thomas et al. [38], is a elementbased local projection framework for splines that allows us to do exactly this. In Bézier extraction, we perform a local projection of the spline basis onto the Bernstein basis via an extraction operator, $\mathbf{C}^{e}$. In Bézier projection, we instead project any function onto a spline basis, and as a result, we can approximate any geometry as a spline object. More specifically, we are interested in projecting the global NURBS or T-spline surface onto a collection of piecewise Bézier elements. In this section, we introduce Bézier projection for the univariate case by example. In Section 6.3, we will demonstrate how we extend this to the bivariate case for the purposes of surface reconstruction.

First let us define what we mean by Bézier projection. Let $\hat{\Omega} \subseteq \mathcal{R}^{d_{r}}$ denote a given domain, and let $f: \hat{\Omega} \rightarrow \mathbb{R}^{d_{s}}$ be a given target function to be approximated. For the case of interest, $f$ is the parameterization of the global NURBS or T-spline surface, and $\hat{\Omega}$ is the parametric domain for the surface. We aim then to approximate $f$ using a spline function $f^{h}$ within a set of candidate spline funciton $\mathcal{T}$. There exist many means of doing so, including point collocation and $L^{2}$ projection. Generally speaking, we aim to define a projection operator $\Pi:\left(L^{2}(\hat{\Omega})\right) \rightarrow \mathcal{T}$ such that (1) $\Pi$ is a linear operator, and (2) $\Pi$ is 
spline perserving in that $\Pi f=f$ for all $f \in \mathcal{T}$. That is:

$$
f^{h}=\Pi(f)=\sum_{A} \mathbf{P}_{A}(f) N_{A}
$$

where $\mathbf{P}_{A}$ are the control points of the spline approximation and $N_{A}$ are the spline basis functions $N_{A} \in \mathcal{T}$. We note that if $f \in \mathcal{T}$, then Bézier projection is exact.

It remains then to describe precisely how the spline control points are found. With Bézier preojection, we define the spline control points via a three step process, which we demonstrate below in the univariate setting. Consider the function $f(\xi)=[\xi, \sin (\xi)+\xi], \xi \in$ $[0,3 \pi]$ shown in Figure 9a. We desire to project the spline onto the B-spline curve defined over the knot vector $\Xi=\left\{\begin{array}{lllll}0 & 0 & 0 & 0\end{array} \pi \pi 2 \pi 2 \pi 2 \pi 3 \pi 3 \pi 3 \pi 3 \pi\right\}$. The process for Bézier projection is then carried out as follows:

\section{Local projection onto the Bernstein Basis}

First, the domain $\hat{\Omega}$ is partitioned into $N$ elements, $\hat{\Omega}^{e} \subseteq \hat{\Omega}$, where the domain $\hat{\Omega}^{e}$ corresponds to the domain of a local Bézier curve. For each subdomain, there exists a mapping $\phi^{e}:\left[\begin{array}{ll}0 & 1\end{array}\right] \rightarrow \hat{\Omega}^{e}$ that maps the unit interval to the element $e$. Then, we find the local Bézier control points, $\mathbf{P}^{b, e}$ by solving the system:

$$
\sum_{j=0}^{p}\left(B_{i}, B_{j}\right) \mathbf{P}_{j}^{b, e}(f)=\left(B_{i}, f \circ \phi^{e}\right) \quad i=\{0, \ldots, p\}
$$

where $(f, g)$ denotes the $L_{2}$ inner product over the unit interval, defined as:

$$
(f, g)=\int_{0}^{1} f(\xi) g(\xi) d \xi
$$

Note that this yields the $L^{2}$ projection of $f$ onto the space of Bernstein polynomials. Thus the local projection of $f$ onto the Bézier curve is given by:

$$
\Pi^{e}(f)=\sum_{i=0}^{p} \mathbf{P}_{i}^{b, e}(f) B_{i}
$$

Figure $9 \mathrm{~b}$ shows the result of local projection of $f$ onto three Bézier curves.

\section{Projection of local Bézier values onto local NURBS values.}

Once we have determined the local Bézier control points for each element, we must project these values onto the NURBS control points. To do so, we employ the element reconstruction operator, which maps the Bernstein basis into the global spline basis for a given element. Recall that in Bézier extraction, the element extraction operator $\mathbf{C}^{e}$ maps the NURBS control points to the Bézier control points. It holds then that $\mathbf{R}^{e}$, can be defined as the inverse of the element extraction operator, $\mathbf{R}^{e}=\left(\mathbf{C}^{e}\right)^{-1}$. Then, to exactly preserve the function $\Pi^{e}(f)$ in terms of the spline basis, it is then sufficient to define the local spline control points as: 


$$
\mathbf{P}^{e}(f)=\left(\mathbf{R}^{e}\right)^{T} \mathbf{P}^{b, e}(f)
$$

\section{Blending of local values to find global values.}

Finally, if $f \notin \mathcal{T}$, the reconstructed NURBS control points will not coincide from element to element, as is readily seen in Figure $9 \mathrm{~b}$. It remains then to somehow select a global control point from the collection of element control points. We note that there exists a wide variety of possible choices for selecting, averaging, or recombining these control points, and of the possibilities, none have been shown to be provably optimal. However, Thomas et al. propose a weighted averaging scheme based on element size, that has been demonstrated to yield good results. The global NURBS control points are found from the local NURBS control point points via the relation:

$$
\mathbf{P}_{A}(f)=\sum \omega_{A}^{e} \mathbf{P}_{A}^{e}(f)
$$

where the weights, $\omega_{A}^{e}$, are defined as:

$$
\omega_{A}^{e}=\frac{\int_{\Omega^{e}} N_{A} d \Omega}{\sum_{e^{\prime}=E_{A}} \int_{\Omega^{e^{\prime}}} N_{A} d \Omega}
$$

Figure $9 \mathrm{c}$ shows the resulting global NURBS curve approximation of $f$.

With our description of the Bézier projection process concluded, we take a moment to comment briefly on a few more practical considerations. First, we note that much like with Bézier extraction, when working with a rational spline basis, it is required to first transform the spline to projective space, and perform projection there. Also, as with Bézier extraction, Bézier projection extends readily to the multivariate case. Finally, we note that for the example considered here, and the applications considered in this paper, we desire to simply project onto the piecewise Bernstein basis. As such, the projection operation on the local Bézier control points simply has the effect of performing a direct mapping from local Bézier control points to local spline control points. Indeed, it can be verified that for the knot vector considered in this example that the element extraction operators, and therefore the element reconstruction operators, are all simply the identity matrix.

\section{Geometric Polynomial Complexity}

Before proceeding forward to a discussion on mesh generation, it is useful to introduce one more fundamental concept. When dealing with higher-order surfaces, it is useful to define some notion of the total (rational) polynomial complexity of the surface. That is, we would like to define an upper bound on the polynomial degree needed to exactly represent the surface. Recall from Section 4.2 that Bézier projection is exact for a target function $f$ if $f \in \mathcal{T}$. Thus, if we can determine the polynomial complexity of the surface, we can then determine what polynomial degree elements are needed to create a geometrically exact mesh of the object. With this in mind, we introduce the following two definitions. 


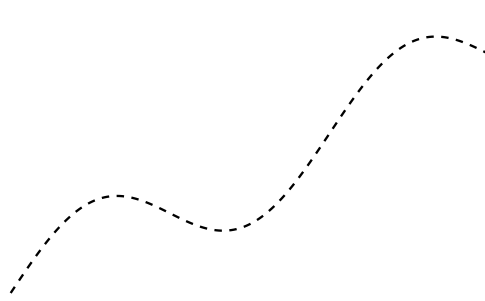

(a)

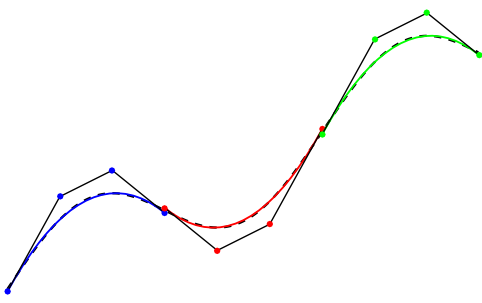

(b)

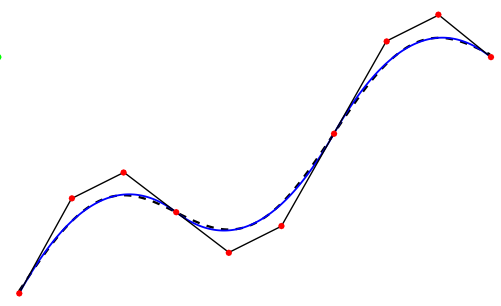

(c)

Figure 9: (a) The target function, $f$. (b) Local projection of $f$ onto three Bézier curves. The three Bézier curves are shown in different colors along with their control nets. (c) The NURBS approximation to $f$.

Definition 5.1. $P_{1}$ Complexity

A geometry $\Omega \subset \mathbb{R}^{d_{s}}$ is said to have polynomial complexity, or $P_{1}$-complexity, of degree $p$ if the following hold:

1. There exists a set of non-overlapping open sets, $\Omega^{e}$, such that $\bar{\Omega}=\overline{\bigcup_{e=1}^{n} \Omega^{e}}$

2. For each open set $\Omega^{e}$, there exists a bijective polynomial or rational ${ }^{4}$ map of degree $p, \vec{x}_{e}: \hat{\Omega}^{e} \rightarrow \Omega^{e}$, where $\hat{\Omega}^{e}$ is an open set $\hat{\Omega}^{e} \subset \mathbb{R}^{d_{r}}$.

3. For each pair $\Omega^{i}$ and $\Omega^{j}$, where $i \neq j$, and $\partial \Omega^{i} \cap \partial \Omega^{j} \neq \emptyset$, the transition mapping, $\vec{t}_{i j}: \hat{\Gamma}_{i j} \rightarrow \hat{\Gamma}_{j i}$, defined by:

- $\vec{t}_{i j}:=\vec{x}_{j}^{-1} \circ \vec{x}_{i}$

- $\hat{\Gamma}_{i j}:=\vec{x}_{i}^{-1}\left(\partial \Omega^{i} \cap \partial \Omega^{j}\right)$

- $\hat{\Gamma}_{j i}:=\vec{x}_{j}^{-1}\left(\partial \Omega^{i} \cap \partial \Omega^{j}\right)$

is affine.

Definition 5.2. $P_{2}$ Complexity

A geometry $\Omega \subset \mathbb{R}^{d_{s}}$ is said to have polygonal polynomial complexity, or $P_{2}$-complexity, of degree $p$ if the above hold and the open sets $\hat{\Omega}^{e}$ are polygons.

It should be noted that our definitions for $P_{1}$ and $P_{2}$ complexity accommodate for rational maps in addition to polynomial maps. This is important as many geometric objects of engineering interest including conic sections cannot be represented using polynomial maps of finite degree. To illustrate the notion of polynomial complexity, we have included a collection of surfaces with different $P_{2}$ complexity in Figure 10. It remains to establish a simple computational test to determine the $P_{2}$ complexity of a surface. As it turns out, such a test is not readily available. However, recall that for any given NURBS or T-Spline surface, we can define the surface as a collection of Bézier quadrilaterals obtained through

\footnotetext{
${ }^{4}$ We identify the polynomial degree of a rational map as the maximum of the degrees of the numerator and denominator.
} 
Bézier extraction. Each Bézier quadrilateral is represented in terms of a parameterization $\mathbf{x}^{\text {quad }}: \hat{\Omega}^{\text {quad }} \rightarrow \mathbb{R}^{3}$. While we cannot directly measure the geometric complexity of the surface, we can in fact design a test to measure the complexity of the parameterization. To design such a test, we first consider the transformation from the bivariate Bernstein basis to the power basis, which is defined as the complete set of polynomials of degree $p$. For example, the bi-cubic power basis is defined as:

$$
P=\left[1, \xi_{1}, \xi_{2}, \xi_{1}^{2}, \xi_{2}^{2}, \xi_{1} \xi_{2}, \xi_{1}^{3}, \xi_{2}^{3}, \xi_{1}^{2} \xi_{2}, \xi_{1} \xi_{2}^{2}, \xi_{1}^{3} \xi_{2}, \xi_{1} \xi_{2}^{3}, \xi_{1}^{2} \xi_{2}^{2}, \xi_{1}^{3} \xi_{2}^{2}, \xi_{1}^{2} \xi_{2}^{3}, \xi_{1}^{3} \xi_{2}^{3}\right]
$$

We then define the transformation matrix $\mathbf{T}$, which defines the transformation from the power basis to the Bernstein basis through the relation:

$$
B_{i}=\sum_{j} \mathbf{T}_{i j} P_{j}
$$

This allows us to define the set of homogeneous power basis coefficients $\left\{\widetilde{\mathbf{P}}_{i}^{p}\right\}_{i=1}^{n}$ from the homogeneous Bézier control points $\left\{\widetilde{\mathbf{P}}_{i}^{b}\right\}_{i=1}^{n}$, via the relation:

$$
\widetilde{\mathbf{P}}^{p}=(\mathbf{T})^{T} \widetilde{\mathbf{P}}^{b}
$$

The power basis coefficients $\left\{\mathbf{P}_{i}^{p}\right\}_{i=1}^{n}$ and their corresponding weights $\left\{w_{i}^{p}\right\}_{i=1}^{n}$ can then be found as:

$$
\begin{array}{r}
\left(\mathbf{P}_{i}^{p}\right)_{j}=\left(\widetilde{\mathbf{P}}_{i}^{p}\right)_{j} /\left(\widetilde{\mathbf{P}}_{i}^{p}\right)_{d_{s}+1} \quad i \in\{1, \ldots, n\}, j \in\left\{1, \ldots, d_{s}\right\} \\
w_{i}^{p}=\left(\widetilde{\mathbf{P}}_{i}^{p}\right)_{d_{s}+1}
\end{array}
$$

It can be verified that the Bézier surface can then be defined in terms of the power basis and corresponding coefficients in much the same way as it can using the Bernstein basis and Bézier control points. That is:

$$
\vec{x}^{\text {quad }}(\boldsymbol{\xi})=\frac{\sum_{i} P_{i}(\boldsymbol{\xi}) \mathbf{P}_{i}^{p}}{\sum_{j} P_{j}(\boldsymbol{\xi}) w_{j}^{p}}
$$

Of course, the power basis coefficients give very little intuition to the shape of the surface, which is why they are not used in the geometric modeling context. However these coefficients do give insight into the polynomial degree of the parameterization. It can be readily seen from Equation (57) that any polynomial degree that is not needed to parameterize the surface will have a corresponding power basis coefficient $\mathbf{P}_{i}^{p}=\left[\begin{array}{lll}0 & 0 & 0\end{array}\right]$. In this way, we establish a simple, computationally efficient test for the $P_{2}$ complexity of a parameterization. The transformation matrix $\mathbb{T}$ is defined only as a function of the two bases, and needs only be computed once. Then, for each Bézier element, the power basis coefficients, and therefore the $P_{2}$ complexity of the parameterization, can be found via Equation (55).

Finally, we must clarify a somewhat subtle aspect of $P_{2}$ complexity, namely the difference between the $P_{2}$ complexity of the geometry, and the $P_{2}$ complexity of the parameterization. 


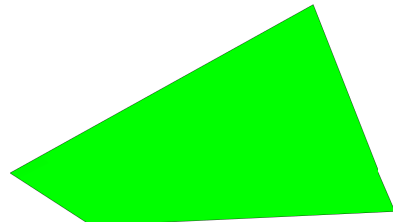

(a)

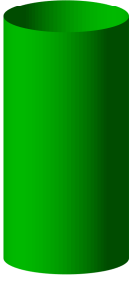

(b)

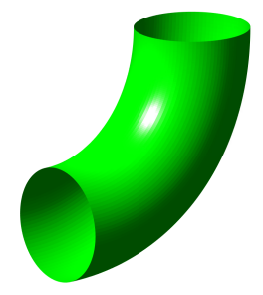

(c)

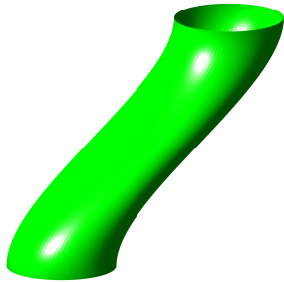

(d)

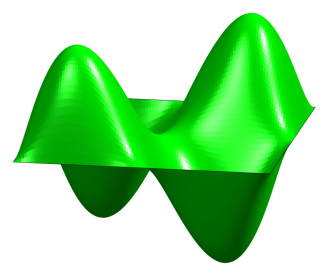

(e)

Figure 10: A variety of surfaces with different $P_{2}$ complexity: (a) Bilinear surface, $P_{2}=2$, (b) Cylinder, $P_{2}=3$, (c) Pipe Elbow, $P_{2}=4$, (d) S-Duct, $P_{2}=5$, (e) Bi-cubic surface, $P_{2}=6$.

The test for $P_{2}$ complexity outlined in Equation (55) will yield the $P_{2}$ complexity of the parameterization, which is not necessarily equal to the $P_{2}$ complexity of the geometry. For example, consider that it is possible to parametrize a circle by a collection of $C^{0}$-continuous quadratic Bézier curves, or by a single $C^{1}$-continuous quartic rational Bézier curve [9]. In both cases, the $P_{2}$ complexity of the geometry is $P_{2}=2$. However, the $P_{2}$ complexity of the $C^{0}$-continuous quadratic parameterization is $P_{2}=2$, while the polynomial complexity of the $C^{1}$-continuous quartic parameterization is $P_{2}=4$. It is useful to note that for any given geometry, the $P_{2}$ complexity of the parameterization serves as an upper bound for the $P_{2}$ complexity of the geometry. While it is beyond the scope of this work, it could be both interesting and useful in future work to develop a direct test of the $P_{2}$ complexity of the geometry, as well as developing an algorithm to re-parameterize the geometry to the lowest complexity parameterization.

\section{Higher Order Mesh Construction}

With the relevant background introduced, we now turn our attention to the main focus of this work: construction of higher-order, geometrically exact meshes. Generally speaking the procedure is broken into four steps:

Step 1: Generation of a compatible linear mesh.

Step 2: Degree elevation of the linear mesh.

Step 3: Surface reconstruction.

Step 4: Smoothing of control points and weights.

The rest of this section will provide detailed descriptions of each of the steps listed above. 


\subsection{Generation of a Compatible Linear Mesh}

The focus of this work is not on linear mesh generation technologies, and we do not propose any new methods for mesh generation in this paper. Instead, we rely heavily on existing mesh generation technologies, and this section serves to outline our requirements for the initial linear mesh. In traditional linear mesh generation, the meshing process begins by first creating a polygonal surface mesh, and a polyhedral volumetric mesh is created based off of this surface mesh. Since CAD geometries are encoded by surfaces, we are specifically interested in ensuring that we generate suitable linear surface meshes such that we are able to reconstruct the geometry in a later step. In order to ensure that this is possible, we impose several constraints on both the CAD surface and resulting linear surface mesh.

First, let the CAD surface be comprised of any number of non-intersecting, orientable, closed manifolds without boundary. For each manifold, $\mathcal{S}$, we require that the manifold be explicitly parameterized by a watertight ${ }^{5}$ NURBS or T-spline surface. Thus for each manifold, we can define a set of Bézier elements $\Omega^{e}$ through Bézier extraction such that:

$$
\overline{\mathcal{S}}=\overline{\bigcup_{e=1}^{n} \Omega^{e}}
$$

and for each Bézier element there exists a rational Bernstein-Bézier mapping $\vec{x}^{e}: \hat{\Omega}^{\text {quad }} \rightarrow$ $\Omega^{e}$. Thus the extracted elements provide an explicit, bijective, watertight parameterization of $\mathcal{S}$.

Once we have ensured that the CAD surface is valid, we must generate a suitable linear mesh of the surface. We call such a surface mesh compatible, and the requirements for a compatible mesh are roughly stated as follows:

Requirement 1: Each vertex in the mesh is a point on the surface $\mathcal{S}$.

Requirement 2: Each polygon in the mesh belongs to a unique Bézier element.

To make these requirements precise, we denote a linear surface mesh as $\mathcal{M}=\{\mathcal{V}, \mathcal{P}\}$, where $\mathcal{V}$ is the set of vertices in the mesh, and $\mathcal{P}$ is a polygonization of the vertices in $\mathcal{V}$. Requirement \#1, mathematically speaking, requires that $V \in \mathcal{S}$ for every vertex $V \in \mathcal{V}$. Requirement $\# 2$, on the other hand, requires that each vertex for a given polygon $p_{k}^{e} \in \mathcal{P}$ must lie on a unique Bézier element $\Omega^{e} \subset \mathcal{S}$. To illustrate this second requirement, Figure 11a shows an example of a compatible mesh, while Figure 11b shows an incompatible mesh, with the incompatible polygons highlighted in red. The motivation for this second requirement will become clear in Section 6.3, as it ensures that we will be able to perform Bézier projection in order to recover the exact geometry. Again, we note that there exists a wide variety of well established linear mesh generation algorithms [8, 14, 19]. Any one of these algorithms may be used produce the preliminary linear mesh, providing the above conditions are met.

We must mention that there is one caveat on the precise requirements on the CAD surfaces that we have listed above. Consider the cylindrical surface shown in Figure 12a. As it is, this surface does not satisfy our criteria because it is open at the ends, and is therefore not a manifold without boundary. However, for practical implementation, we

\footnotetext{
${ }^{5}$ By a water tight, we mean that the surface is free from gaps and self-intersections.
} 


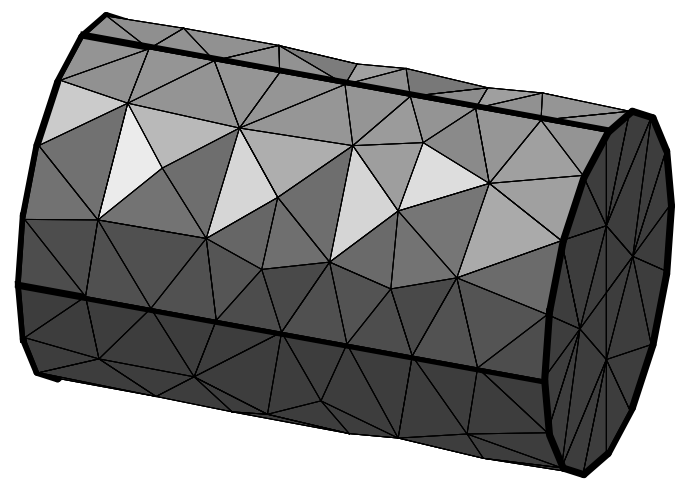

(a)

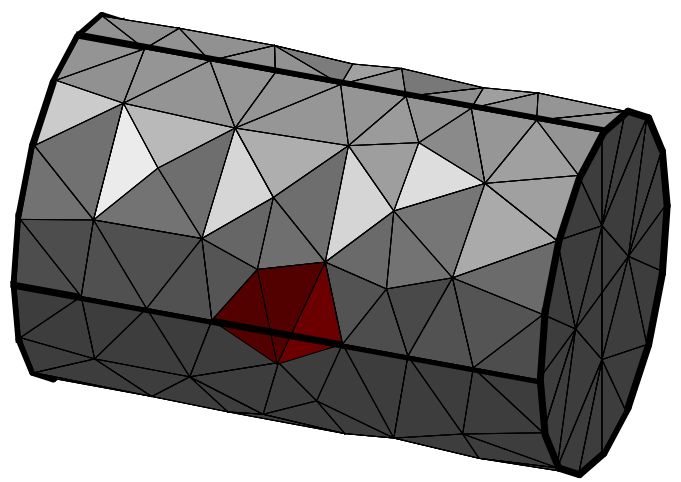

(b) b

Figure 11: a) A valid and (b) an invalid linear mesh. Bézier element boundaries are shown in bold, and invalid triangles are shown in red.

permit the closure of open surfaces using a trimmed surface, provided that the trimmed surface is planar. Figure $12 \mathrm{~b}$ shows the cylinder closed by a trimmed planar surface. The reasoning behind this caveat comes directly from the surface reconstruction constraints. If a surface is planar, we are not required to perform geometry reconstruction, and therefore do not require that the surface be explicitly parameterized by Bézier elements. With a suitable surface mesh generated, it then remains to generate a linear mesh of the volume. Figure 13 shows a cut view of the volume mesh of the cylinder from Figure 12c. The elements intersected by the cutting plane are highlighted in yellow.

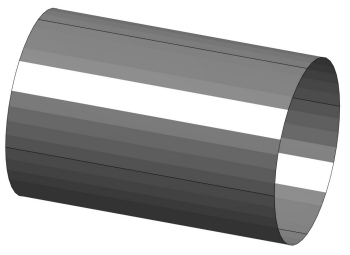

(a)

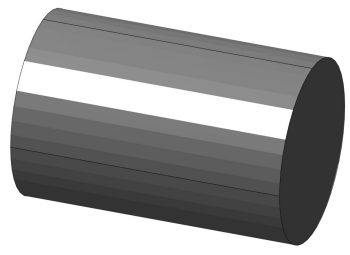

(b)

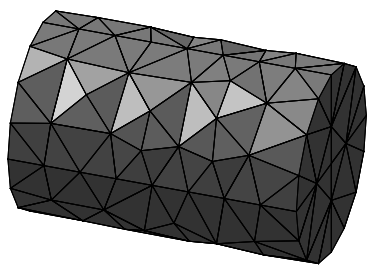

(c)

Figure 12: Triangular surface mesh generation. (a) The initial open cylindrical surface. (b) Closing the surface with a trimmed plane. (c) Generation of an unstructured surface mesh.

\subsection{Degree Elevation of the Linear Mesh}

Once we have generated a suitable linear mesh, we next degree elevate the mesh by locally degree elevating the linear elements to create the control nets for our higher-order Bézier elements. That is, we would like to generate some higher-order Bernstein-Bézier representation of the elements in the linear mesh. To achieve this, let us first denote the set of $n_{e l}$ polyhedrons in the linear mesh as $\left\{\Omega^{p o l y, E}\right\}_{E=1}^{n_{e l}}$. We then define a mapping $\vec{x}^{p o l y, E}: \hat{\Omega} \rightarrow \Omega^{\text {poly }, E}$, that maps the reference polyhedron $\hat{\Omega}$ to the physical polyhedron $\Omega^{\text {poly,E}}$ for each polyhedron in the mesh. Let us also define the equispaced control net over 


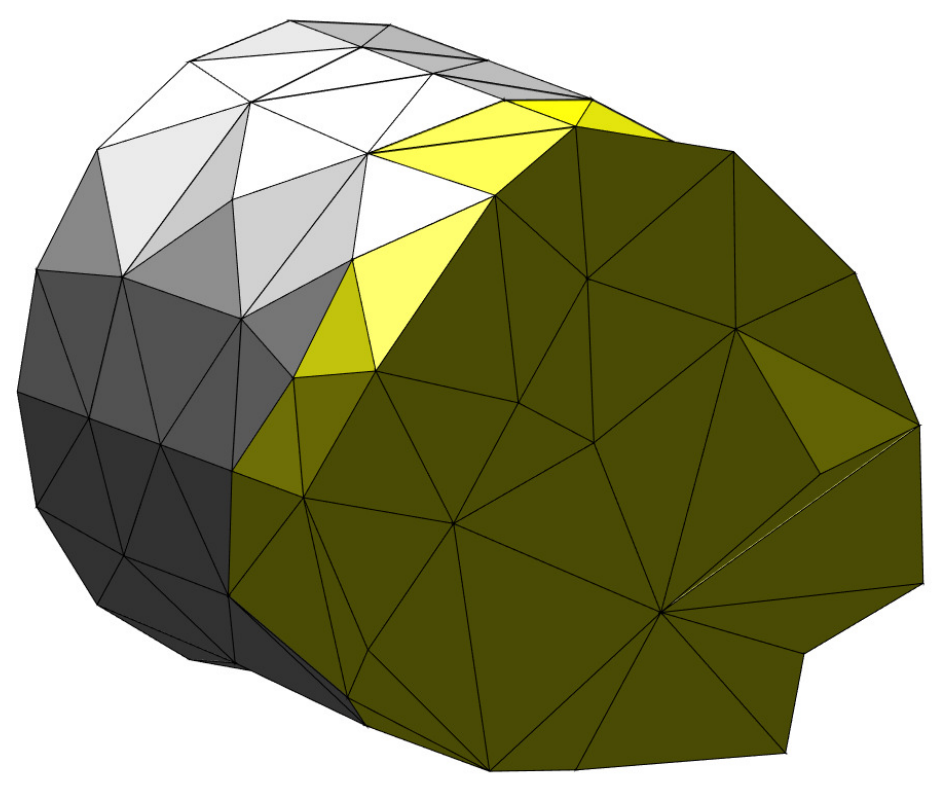

Figure 13: Cut view of the linear cylinder mesh.

the reference polyhedron $\left\{\boldsymbol{\xi}_{i}\right\}_{i \in I} \in \hat{\Omega}$. Examples of such reference control nets are visualized for the cubic geometric primitives in Figure 7 in Section 3. Then, for each polyhedron in the linear mesh, we can define the higher order Bézier control net via the mapping:

$$
\mathbf{P}_{i}^{b, E}=\vec{x}^{p o l y, E}\left(\boldsymbol{\xi}_{i}\right)
$$

We note that the mapping $\vec{x}^{p o l y, E}$ is affine for tetrahedra, bilinear for wedges, and trilinear for hexahedra and pyramids. Finally, during this step, we additionally set all of the control point weights to unity. The result of this process is shown in Figure 14, which displays the degree elevated linear mesh of the cylinder from Figure 13 to a cubic tetrahedral mesh. With this step complete, we have obtained a higher-order mesh, but it remains to encode the geometry from the surface in the mesh. This is achieved through surface reconstruction procedure described in the next section.

\subsection{Surface Reconstruction}

The next step is to update the newly generated control nets so that the mesh surface exactly matches the CAD surface. Recall from Section 4.2 that Bézier projection allows us to project any function onto a NURBS basis. In the context of surface reconstruction, we desire to project the Bézier elements from our CAD surface onto a collection of Bézier quadrilaterals and triangles. However, before we describe the exact process for performing surface reconstruction, we first take a moment to introduce some useful notation. Figure 15 serves to illustrate the notation introduced here. 


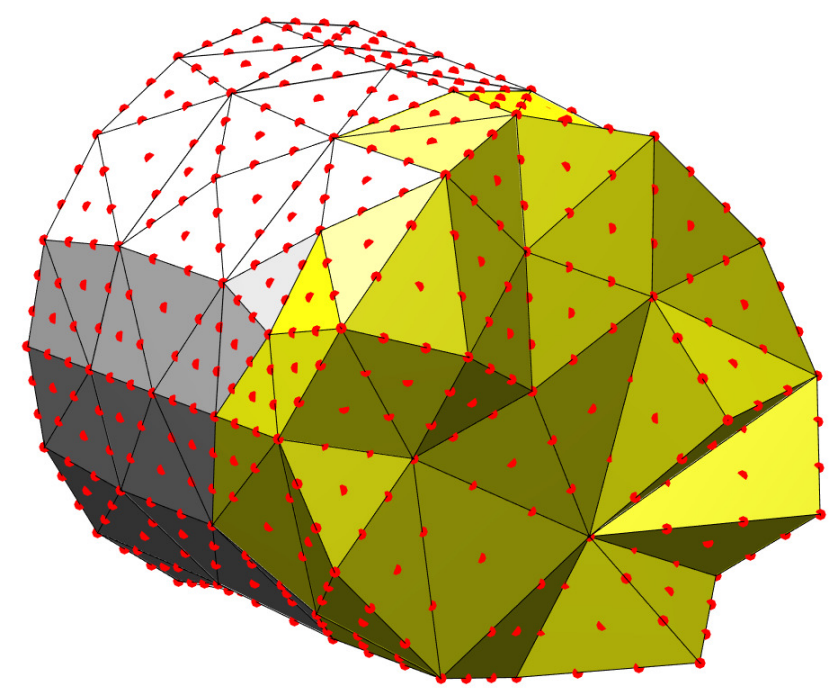

Figure 14: Degree elevated cylindrical mesh.

Recall from Section 6.1 that each polygon in the surface mesh belongs to a unique Bézier element. As a result, we can associate a unique set of polygons, which we denote as $\left\{p_{k}^{e}\right\}_{k=1}^{n}$, with each Bézier element $\Omega^{e}$. Then, for a given polygon $p_{k}^{e}$, we define $\vec{\psi}_{k}^{e}: \hat{\Omega}^{k} \rightarrow p_{k}^{e}$ to be the unique mapping such that:

$$
\vec{\psi}_{k}^{e}\left(\left(\vec{x}^{e}\right)^{-1}(V)\right)=V
$$

for every vertex $\mathrm{V}$ of the polygon $p_{k}^{e}$. This mapping is affine for triangles and bilinear for quadrilaterals. Next, let us define the corresponding parametric polygon $\hat{p}_{k}^{e}$ as:

$$
\hat{p}_{k}^{e}=\left(\vec{\psi}_{k}^{e}\right)^{-1}\left(p_{k}^{e}\right)
$$

Note that the set of parametric polygons $\left\{\hat{p}_{k}^{e}\right\}_{k=1}^{n}$ forms a non-overlapping cover of $\hat{\Omega}^{q u a d}$, meaning:

$$
\begin{aligned}
\overline{\hat{\Omega}^{q u a d}} & =\overline{\cup \overline{\hat{p}_{k}^{e}}} \\
\cap \hat{p}_{k}^{e} & =\emptyset
\end{aligned}
$$

Consequently, $\left\{\hat{p}_{k}^{e}\right\}_{k=1}^{n}$ forms a watertight polygonization of the reference quadrilateral.

Now, let us introduce a few more mappings. Namely, let $\vec{\phi}_{k}^{e}: \hat{\Omega}^{k} \rightarrow \hat{p}_{k}^{e}$ be the unique mapping from the reference polygon $\hat{\Omega}^{k}$ to the parametric polygon $\hat{p}_{k}^{e}$. As with $\vec{\psi}_{k}^{e}$, this mapping is affine for triangles and bilinear for quadrilaterals. With $\vec{\phi}_{k}^{e}$ defined, we can define the composite mapping:

$$
\vec{x}_{k}^{e}:=\vec{x}^{e} \circ \vec{\phi}_{k}^{e}
$$


where $\vec{x}_{k}^{e}$ is a bijective mapping between $\hat{\Omega}^{k}$ and the physical entity $\vec{x}^{e}\left(\hat{p}_{k}^{e}\right)=\Omega_{k}^{e} \subseteq \Omega^{e}$. The set of physical entities $\left\{\Omega_{k}^{e}\right\}_{k=1}^{n}$ form a non-overlapping cover of $\Omega^{e}$, and thus our goal is to construct a Bernstein-Bézier representation of each mapping $\vec{x}_{k}^{e}: \hat{\Omega}^{k} \rightarrow \Omega_{k}^{e}$. That is, for each entity $\Omega_{k}^{e}$, we would like to find a representation of the form $\Omega_{k}^{e}=\sum_{i \in I} R_{i}(\boldsymbol{\xi}) \mathbf{P}_{i}^{b, k}$, where $\left\{\mathbf{P}_{i}^{b, k}\right\}_{i \in I}$ are the set of Bézier control points for $\Omega_{k}^{e}$.

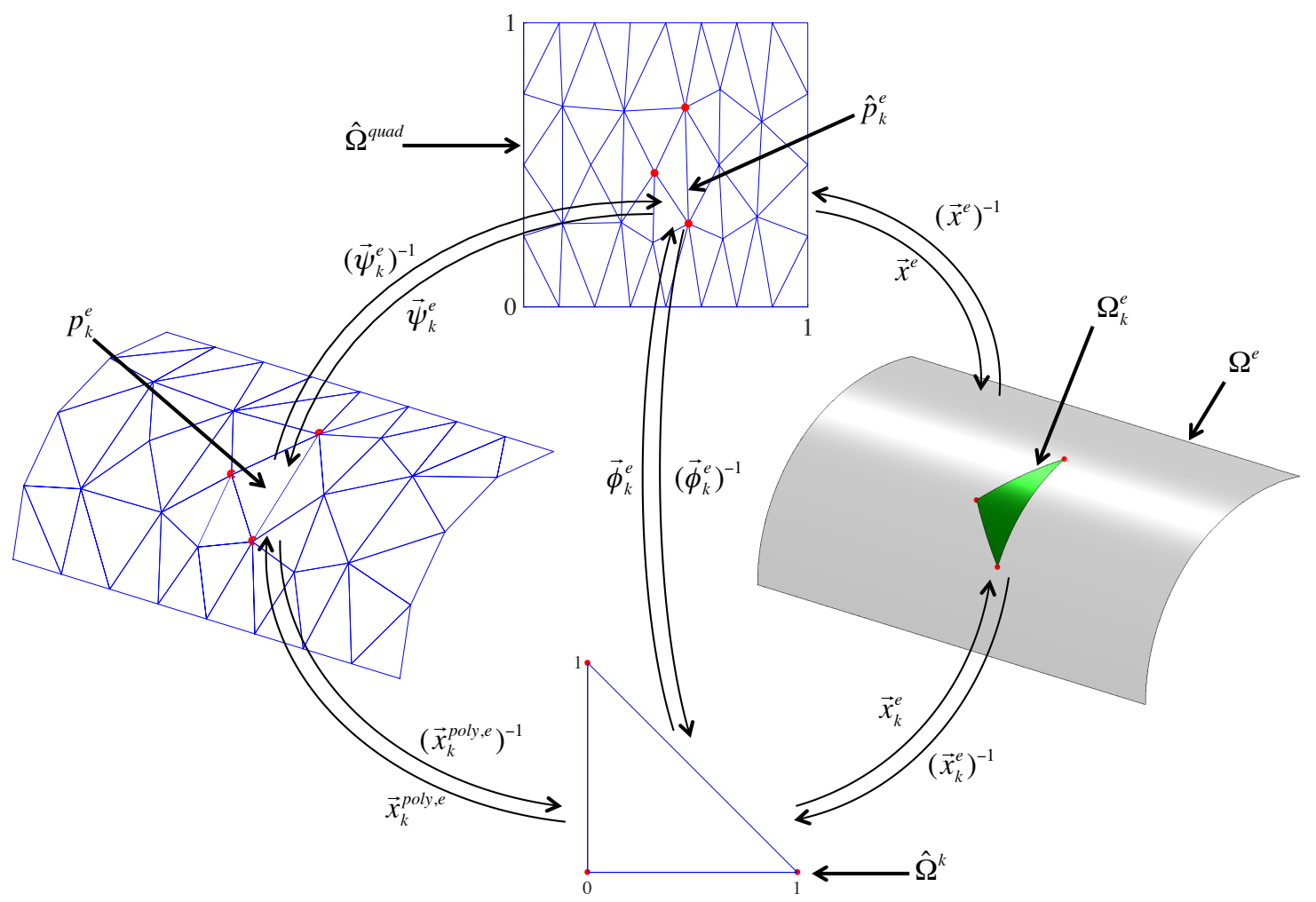

Figure 15: Visualization of Bézier projection of a Bézier element onto a collection of Bézier polygons.

Thus, it remains to provide a method to find the Bézier control points for the surface elements. Let us denote the control points of the Bézier element from the CAD surface as $\mathbf{P}_{j}^{b, e}$. Let us also denote the Bernstein basis functions defined over the unit reference polygon as $\left\{B_{i}^{k}\right\}_{i \in I}$ and the basis functions defined over the unit reference quadrilateral as $\left\{B_{i}^{\text {quad }}\right\}_{i \in I}$. Then, following Bézier projection, we can then find the control points $\left\{\mathbf{P}_{i}^{b, k}\right\}_{i \in I}$ by the relation:

$$
\mathbf{P}^{b, k}=\mathbf{M}^{-1} \mathbf{T} \mathbf{P}^{b, e}
$$

where $\mathbf{M}_{i j}$ is defined as:

$$
\mathbf{M}_{i j}=\int_{\hat{\Omega}^{k}} B_{i} B_{j} d \hat{\Omega}^{k}
$$


and $\mathbf{T}_{i j}$ is defined as:

$$
\mathbf{T}_{i j}=\int_{\hat{\Omega}^{k}} B_{i}\left(B_{j}^{q u a d} \circ \vec{\phi}_{k}^{e}\right) d \hat{\Omega}^{k}
$$

In the case when the Bézier element $\Omega^{e}$ is rational, we proceed similarly, but enforce Equation (65) on the homogenous control points, viz.:

$$
\widetilde{\mathbf{P}}^{b, k}=\mathbf{M}^{-1} \mathbf{T} \widetilde{\mathbf{P}}^{b, e}
$$

Once we have successfully reconstructed the surface, we simply replace the control points that lie on the surface of the degree elevated linear mesh with the control points obtained through surface reconstruction. We likewise update the corresponding control point weights with the weights obtained through surface reconstruction. Figure 16 shows the mesh of the cylinder displayed in Figure 14 after surface replacement.

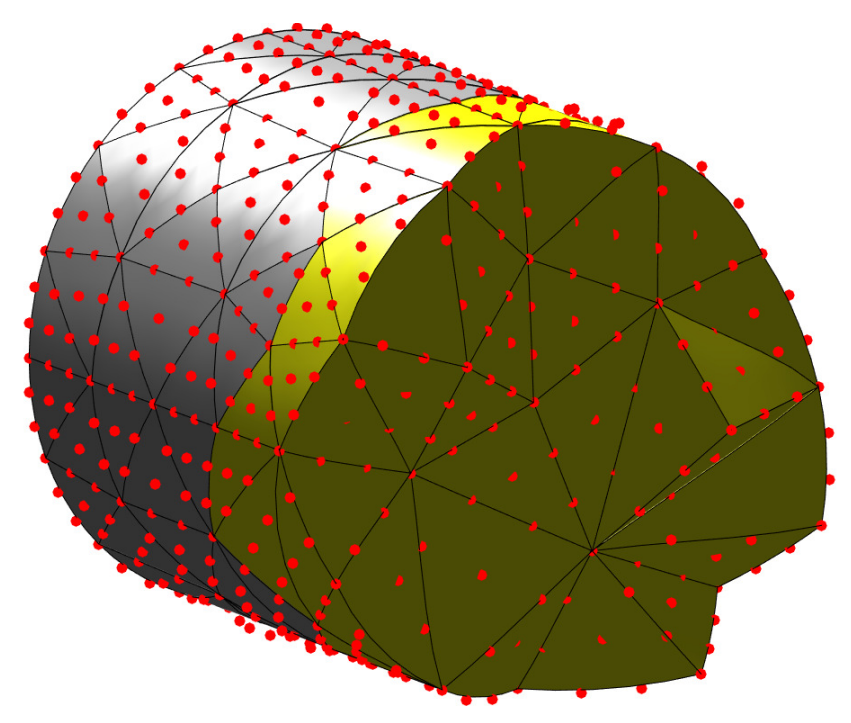

Figure 16: Surface reconstructed cylindrical mesh.

\subsection{Smoothing of Control Points and Weights}

At this point we have succeeded in our original goal of performing isogeometric degree elevation of our mesh. However, we note that the process of surface reconstruction can often lead to large distortion of certain elements. Section 9 provides a more detailed discussion of mesh quality metrics, but for now we present a method for smoothing the internal control point locations and weights in the reconstructed mesh. We have found that in many cases, this final smoothing step can help to improve mesh quality, so we present it here as a useful tool in the meshing process.

When the control point weights are not all identically unity, we begin by solving a Laplace smoothing problem to smooth the control point weights on the interior of the 
mesh. Let $\Omega$ denote the degree-elevated, non-reconstructed mesh, and let $w_{s}$ denote the weighting function associated with the surface obtained during the surface reconstruction process. Then, let $\mathcal{V}^{h}$ be the set of candidate weighting functions that satisfy $\left.w^{h}\right|_{\Gamma}=w_{s}$. We can then smooth the interior control point weights $w^{h}$ by solving the minimization problem:

$$
\min _{w^{h} \in \mathcal{V}^{h}} \frac{1}{2} \int_{\Omega}\left|\nabla w^{h}\right|^{2} d \Omega
$$

We can similarly smooth the internal control point locations by solving a linear elasticity problem. Again, let $\Omega$ denote the degree-elevated, non-reconstructed mesh, and let $\vec{d}_{s}$, denote the displacement of the surface nodes under the surface reconstruction process. We define $\mathcal{V}^{h}$ to be the set of candidate displacement functions that satisfy $\left.\overrightarrow{d^{h}}\right|_{\Gamma}=\vec{d}_{s}$. Note that $\mathcal{V}^{h}$ is a set of rational functions, with respect to the control point weights obtained in the weight smoothing step. We can then smooth the interior control point locations by solving the following linear elasticity problem for the control point displacement, $\overrightarrow{d^{h}}$ :

$$
\min _{\overrightarrow{d^{h}} \in \overrightarrow{\mathcal{V}}^{h}} \frac{1}{2} \int_{\Omega} \boldsymbol{\epsilon}\left(\overrightarrow{d^{h}}\right) \mathbf{D} \boldsymbol{\epsilon}\left(\overrightarrow{d^{h}}\right) d \Omega
$$

where $\mathbf{D}$ and $\boldsymbol{\epsilon}$ are the elasticity tensor and strain vector, respectively.

\subsection{Applications to Higher-Order FEA}

We take this opportunity to discuss the nature of exact geometry. Recall from section 4.2 that Bézier projection is exact if and only if $\vec{x}_{k}^{e} \in \mathcal{T}$. Thus in order to guarantee that our surface reconstruction process is exact, we must ensure that the polynomial degree of our mesh is at least as large as the $P_{2}$ complexity of the parameterization given by $\vec{x}^{e}$. That is, if our parameterization has a $P_{2}$ complexity of $P_{2}=6$, we require sextic triangles on the surface to exactly match the geometry. Additionally, we note that, generally speaking, the mapping $\vec{\phi}_{k}^{e}$ for a quadrilateral is bilinear. As a result the composition of the mappings

$\vec{x}_{k}^{e}=\vec{\phi}_{k}^{e} \circ \vec{x}^{e}$ has twice the polynomial degree of the mapping $\vec{x}^{e}$. Thus we would require dodecic quadrilaterals to exactly match the geometry!

It would appear at this point that we have reached an impasse, as dodecic, or even sextic elements are often impractical for simulation applications. However, we briefly note here two alternatives. First, we recall that even in the case that Bézier projection is not exact, it will still yield a curve or surface of best fit. Thus, it is still possible to use Bézier projection to perform surface reconstruction as we have presented it in Section 6.3. While this surface reconstruction might not be truly isogeometric, it does provide a best fit to the CAD surface, and it is certainly more accurate than the linear mesh. Additionally, despite not being exact, we still recover an unstructured Bernstein-Bézier representation of the volume. We can then use these lower-order meshes to perform an isoparametric FEA simulation on the geometry of interest.

Another possible method for reducing the computational complexity is to use a superparametric representation as is sometimes employed in traditional FEA. In a superparametric approach, the geometry is represented by a basis that is of a higher polynomial degree 
than the basis used to represent the simulation variables. For example, one could employ a discretization of sextic Bernstein-Bézier elements to represent the geometry but use cubic Bernstein polynomials as the basis for FEA.

There are varying advantages and disadvantages of the approaches listed above. Lower order mesh elevation can be computationally less intensive, but suffers from one of the main drawbacks of traditional mesh generation: the initial mesh is still just an approximation, and does not encode the exact geometry. As a result, the geometric approximation error is "set" at the coarsest level of mesh generation. Under mesh refinement, the geometric accuracy cannot be increased without querying the CAD model. The superparametric approach on the other hand does not suffer from this drawback. Because the initial mesh is exact, it can be refined without subsequent queries to CAD. Additionally, a superparametric simulation is less computationally intensive than a corresponding isogeometric simulation but is more computationally intensive than a lower-order isoparametric simulation. In either case, it is most important to note that one of the main advantages to our approach is that it allows for a great deal of flexibility in both mesh generation and simulation. We demonstrate this in Section 10 by providing numerical examples of purely isogeometric simulations, as well as superparametric and lower-order isoparametric simulations.

\subsection{Construction of Structured Surface Meshes}

Before concluding this section, we take a moment to make a quick aside regarding structured surface meshes. While surface meshes of this type form a very restricted subset of all surface meshes, they arise frequently when considering applications such as boundary layer meshing or hex-dominant meshing. As such, it is useful to briefly make special mention of this subset of meshes. Consider the surface mesh of the cylinder shown in Figure 17. The cylindrical surface shown in Figure 17a is refined via knot insertion, resulting in the surface shown in Figure 17b. The surface can then be degree reduced to form a surface mesh comprised of linear quadrilaterals, as shown in Figure 17c.

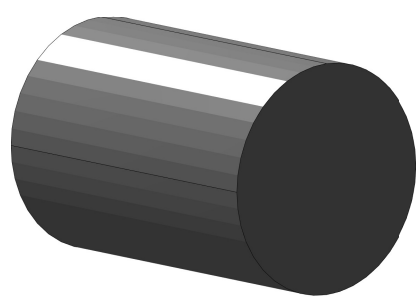

(a)

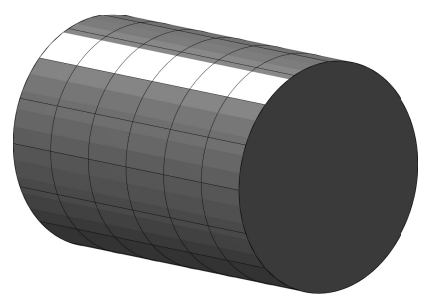

(b)

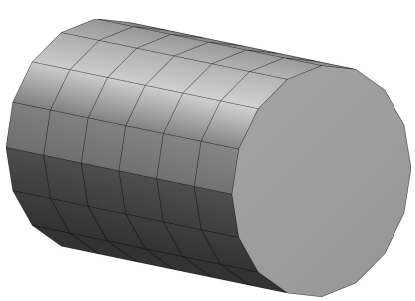

(c)

Figure 17: Structured surface mesh generation. (a) Start with a closed surface. (b) Refine the Bézier surface using Bézier extraction. (c) Degree reduce to arrive at a structured quadrilateral surface mesh.

In the case when the surface mesh over a Bézier element is structured, the surface reconstruction step is much simpler. Since the linear mesh is derived from a refined surface, for each quadrilateral on the surface, there exists an extraction operator $\mathbf{C}^{k}$ such that: 


$$
\widetilde{\mathbf{P}}^{b, k}=\left(\mathbf{C}^{k}\right)^{T} \widetilde{\mathbf{P}}^{b, e}
$$

It is therefore a simple task to find the reconstructed surface control points for each element in the structured surface mesh, and reconstruct the original surface. We note that in the case of extraction based structured surface meshes, the reconstructed surface is guaranteed to be geometrically exact. This gives us some confidence in the ability to generate geometrically exact meshes without the use of dodecic elements.

We also note that this highlights one of the key advantages of Bézier pyramids, as they allow us to transition from a structured surface mesh to an unstructured volume mesh, and preserve the exact geometry in the process. However, while mixed element mesh generation may seem like a panacea, offering both geometric exactness and the benefits of unstructured meshing at relatively low polynomial degree, we note that there are some distinct drawbacks to this approach. First, mixed element mesh generation is still a relatively new field, and it will be some time before it reaches the maturity and robustness of tetrahedral mesh generation. Second, refinement, and especially anisotropic refinement, of mixed element meshes is a challenging task and an ongoing topic of research interest. Finally, the use of all-tetrahedral meshes is still much more popular than mixed-element meshes in a number of applications, including computational fluid dynamics. This is largely due to the aforementioned reasons. As such, we have presented both tetrahedral and mixed-element approaches in this paper in hopes to provide a flexible framework capable of handling a wide array of simulation challenges.

Finally, much like Bézier extraction is a special case of Bézier projection, surface reconstruction of structured meshes is simply a special case of the more general unstructured reconstruction procedure. To wit, we will obtain exactly the same results if we use the Bézeir projection method in lieu of the Bézier extraction method to reconstruct a structured surface mesh. However, if we know a priori that we are working with a structured surface mesh, it is desirable to reconstruct the surface using the extraction method, as it is simpler.

\section{$7 \quad$ Element/Mesh Refinement}

Before considering some numerical examples, it remains to briefly touch upon the topic of mesh refinement. Indeed, mesh refinement is a topic as rich and complex as mesh generation itself. There exist a broad array of algorithms for performing both local and global refinement, as well as more advanced refinement techniques, such as adaptive mesh refinement for applications such as shock capturing. We note that it is always possible to simply refine the linear mesh and degree elevate the resulting refined linear mesh, provided that the refined linear mesh meets the requirements outlined in Section 6.1. However, while this approach eases implementation, it can be costly, as we must repeat the degree elevation process each time we refine. Additionally, this method does not guarantee that the parameterization is exactly preserved, which is often undesirable. In light of this, we present in this section the detailed method for performing refinement via uniform subdivision of the coarse mesh. However, we are secure in the knowledge that we are not restricted to only this type of refinement. 
Consider a parent unit polyhedron in parametric space $\hat{\Omega} \subset \mathbb{R}^{d_{r}}$, and the corresponding Bézier polyhedra in physical space defined by $m$ Bézier control points $\mathbf{P}_{i}^{b} \subset \mathbb{R}^{d_{s}} i=$ $1, \ldots, m$. Then, for each subdivision polyhedra defined in parametric space $\hat{\Omega}^{k} \subset \hat{\Omega}$, we seek a corresponding control net in physical space defined by $n$ Bézier control points, $\mathbf{P}_{j}^{b, k} \subset$ $\mathbb{R}^{d_{s}} \quad j=1, \ldots, n$. To compute these control points, we first define two sets of collocation points. Let $\left\{\boldsymbol{\xi}_{l}\right\}_{l=1}^{n} \in \hat{\Omega}$ denote the set of collocation points in the reference polyhedra, and let $\left\{\boldsymbol{\xi}_{l}^{k}\right\}_{l=1}^{n} \in \hat{\Omega}^{k}$ denote the corresponding set of collocation points on the $k^{t h}$ subdivision polyhedron. Then, we can find the control points $\mathbf{P}_{j}^{b, k}$ by solving the system of equations:

$$
\sum_{j=1}^{n} \mathbf{P}_{j}^{b, k} B_{j}\left(\boldsymbol{\xi}_{l}^{k}\right)=\sum_{i=1}^{n} \mathbf{P}_{i}^{b} B_{i}\left(\boldsymbol{\xi}_{l}\right) \quad l=1, \ldots, n
$$

where $B_{j}$ and $B_{j}^{k}$ are the Bernstein basis functions defined over the parent unit polyhedra and subdivision polyhedra, respectively. Rearranging, we can define a more explicit relationship of the form:

$$
\mathbf{P}^{b, k}=\left(\mathbf{M}^{k}\right)^{-1} \mathbf{S} \mathbf{P}^{b}
$$

where $\mathbf{M}_{i j}^{k}=B_{j}\left(\boldsymbol{\xi}_{i}^{k}\right)$ and $\mathbf{S}_{i j}=B_{j}\left(\boldsymbol{\xi}_{i}\right)$. The astute reader will recognize that with this operation, we are simply projecting the parent polyhedra onto the subdivision polyhedra. When dealing with a rational Bézier polyhedra, we simply evaluate Equation (73) on the homogenous control points, viz.:

$$
\widetilde{\mathbf{P}}^{b, k}=\left(\mathbf{M}^{k}\right)^{-1} \mathbf{S} \widetilde{\mathbf{P}}^{b}
$$

For brevity and ease of implementation, we can define a projection matrix for each subdivision element of the form $\mathbf{T}^{k}=\left(\mathbf{M}^{k}\right)^{-1} \mathbf{S}$. Thus, to perform uniform subdivision on any given Bézier element, we must simply define the projection operators. This in turn simply requires defining collocation points at which to evaluate $\mathbf{M}_{i j}^{k}$ and $\mathbf{S}_{i j}$. Figures 18 through 21 shows the collocation points for calculating $\mathbf{S}_{i j}$ and $\mathbf{M}_{i j}^{k}$, for the four geometric primitives discussed in this paper and for the case of cubic Bernstein-Bézier discetizations. Note that the choice of the collocation points is somewhat arbitrary, but the selection of equispaced control points as shown in Figures 18-21 is both simple and yields suitable results. Finally, it is worth recognizing that with this approach, we are not required to subdivide a Bézier element into like elements. For example, uniform subdivision of a pyramid will result in 6 subdivision pyramids, and 4 subdivision tetrahedra. Figure 22 shows the result of performing uniform subdivision on some representative elements.

Finally, we make one last observation on our refinement process. Consider the uniform subdivisions on hexahedra, tetrahedra, and wedges as presented above. The subdivisions of the parent elements will result in a collection of child subdivision elements. Also note that the faces of the parent elements are subdivided into a number of child subdivision faces. The key observation is this: Subdivision of the parent mesh element yields the same subdivision on the face, as would a subdivision of that face. The key consequence of this is that the coincident faces of two neighboring elements will remain coincident after subdivision! This means that we can locally subdivide each element in a mesh, and the resulting mesh will be valid. 


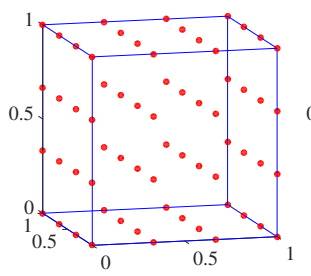

(a)

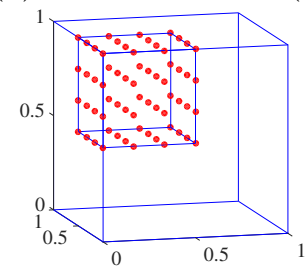

(f)

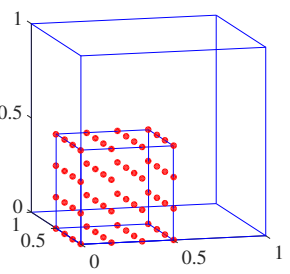

(b)

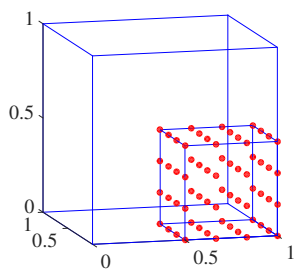

(c)

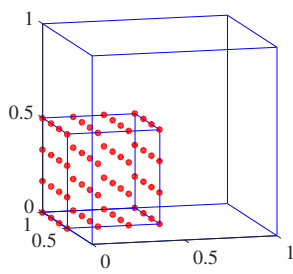

(d)

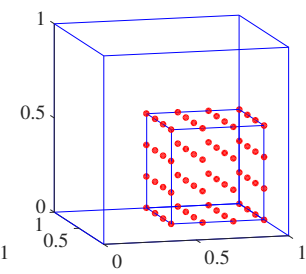

(e)

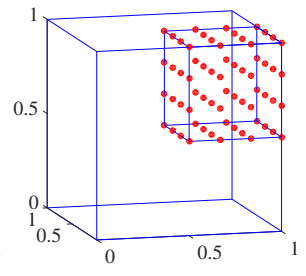

(g)

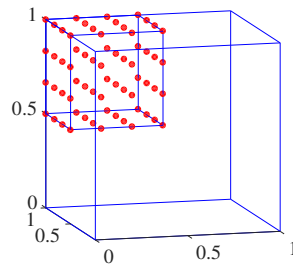

(h)

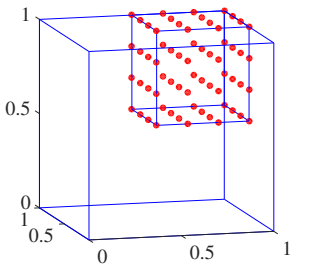

(i)

Figure 18: Refinement of a tricubic Bézier hexahedron. (a) Collocation points for calculating $\mathbf{S}_{i j}$. (b-i) Collocation points for calculating $\mathbf{T}_{i j}^{k}$.

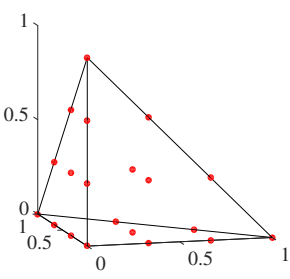

(a)

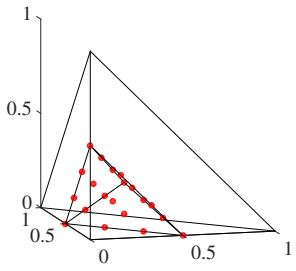

(f)

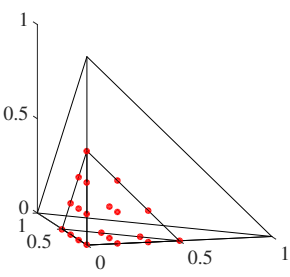

(b)

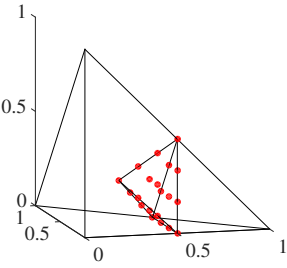

$(\mathrm{g})$

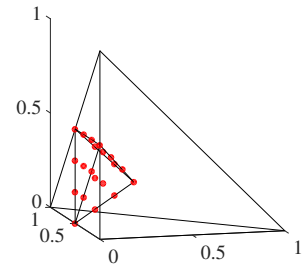

(k)

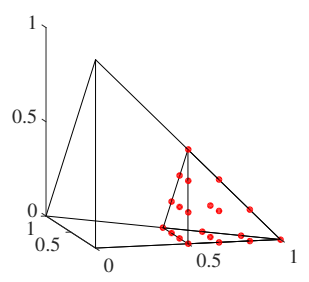

(c)

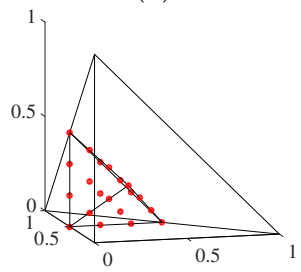

(h)

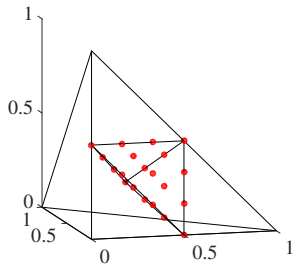

(1)

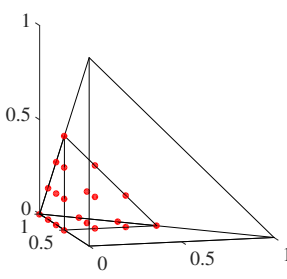

(d)

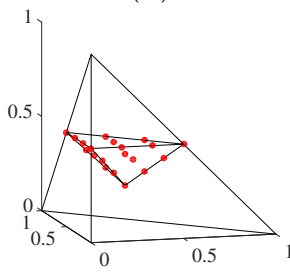

(i)

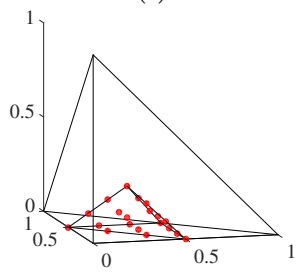

(m)

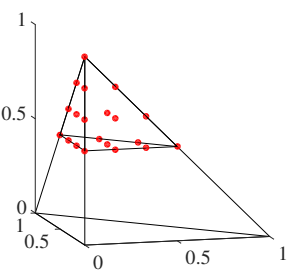

(e)

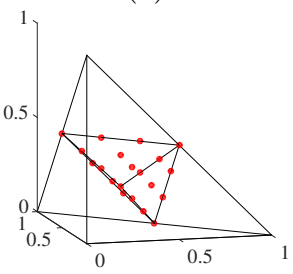

(j)

Figure 19: Refinement of a cubic Bézier tetrahedron. (a) Collocation points for calculating $\mathbf{S}_{i j}$. (b-m) Collocation points for calculating $\mathbf{T}_{i j}^{k}$. We note that uniform subdivision of a tetrahedron results in 4 tetrahedra and an a octahedron in the center. We choose to split this octahedron into 8 tetrahedra as shown above, but other methods of splitting are readily implemented. 


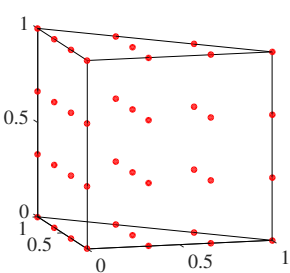

(a)

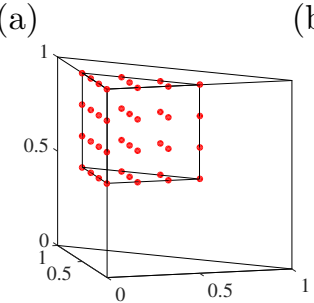

(f)

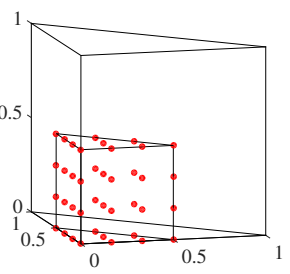

(b)

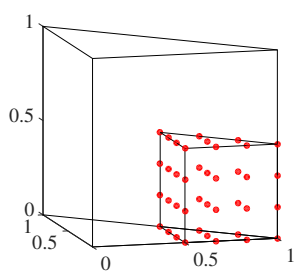

(c)

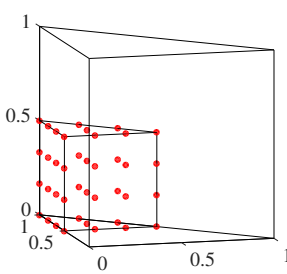

(d)

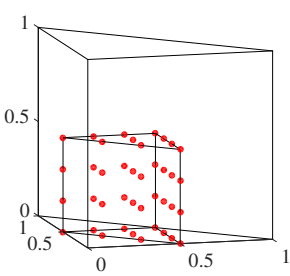

(e)

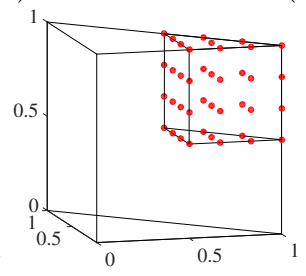

(g)

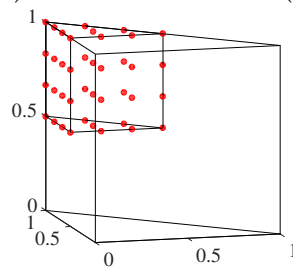

(h)

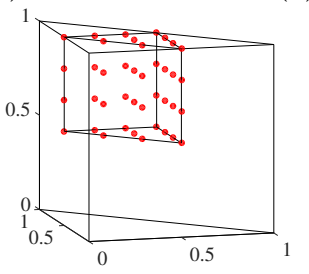

(i)

Figure 20: Refinement of a cubic Bézier wedge. (a) Collocation points for calculating $\mathbf{S}_{i j}$. (b-i) Collocation points for calculating $\mathbf{T}_{i j}^{k}$.

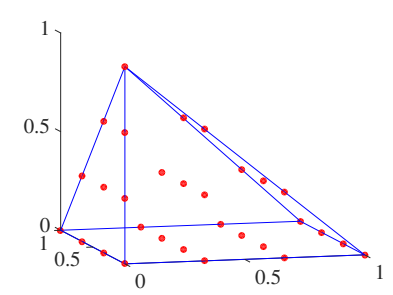

(a)

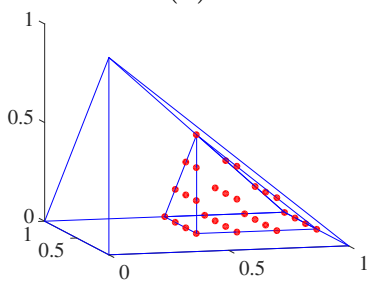

(e)

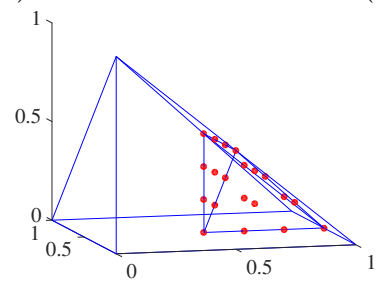

(i)

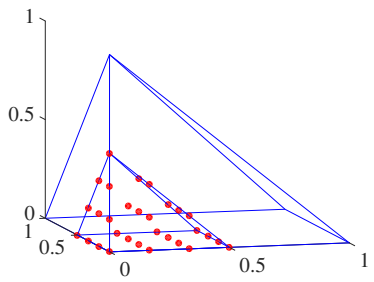

(b)

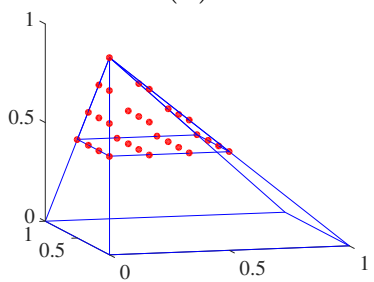

(f)

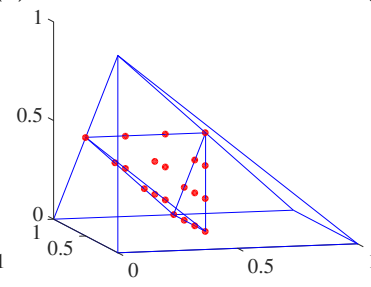

(j)

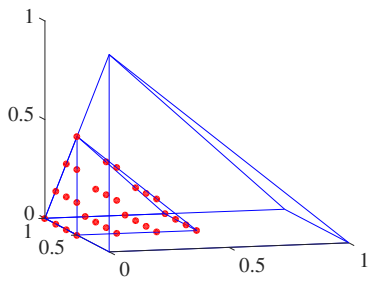

(c)

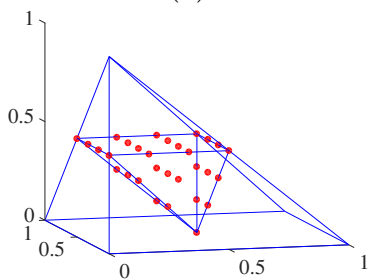

(g)

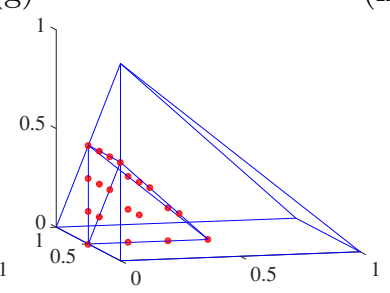

(k)

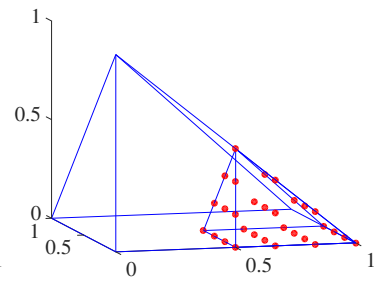

(d)

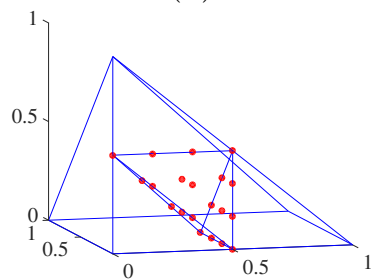

(h)

Figure 21: Refinement of a cubic Bézier pyramid. (a) Collocation points for calculating $\mathbf{S}_{i j}$. (b-k) Collocation points for calculating $\mathbf{T}_{i j}^{k}$. 

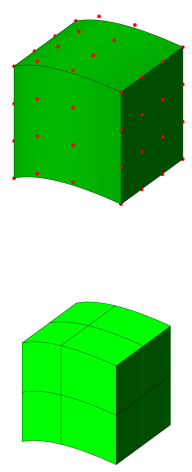

(a)
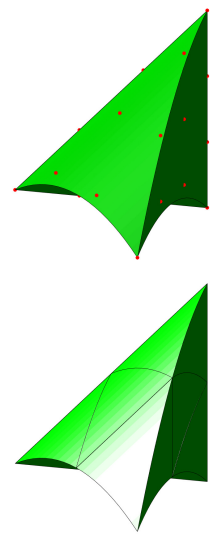

(b)
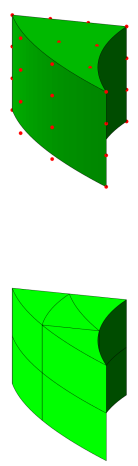

(c)
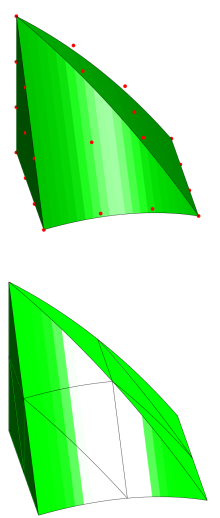

(d)

Figure 22: Uniform subdivision of the meshing primitives.

\section{Mesh Gallery}

With all of the technical details regarding mesh generation covered, we take this opportunity to present a few example meshes generated using the methodology presented in this paper. Figure 23 shows the mesh of a marine propeller, composed of sextic BernsteinBézier tetrahedra. The original T-spline surface is shown in Figure 23a, followed by the surface mesh in Figure 23b. Figure 23c shows a detailed view of the surface mesh with the boundaries of Bézier elements highlighted in bold, and Figure 23d shows a cut cell view of the volumetric mesh. To create the mesh of the propeller, a compatible linear mesh was first created using Tetgen [36]. Then, using our test for $P_{2}$ complexity, we determined the $P_{2}$ complexity of the propeller T-spline surface to be $P_{2}=6$. Finally, we created a geometrically exact mesh composed of sextic Bernstein-Bézier tetrahedra through degree elevation and surface reconstruction.

Figure 24 shows the mesh of a bike frame, composed of cubic Bernstein-Bézier tetrahedra. Again, the T-spline surface is shown in Figure 24a, followed by the surface mesh in Figure 24b, a detailed view in Figure 24c, and a cut cell view in Figure 24d. As with the propeller, a compatible linear mesh was created using Tetgen, and we found the $P_{2}$ complexity of the surface to be $P_{2}=6$. However, to demonstrate the flexibility of our method, we elect instead to create a lower-order mesh of the bike. This is achieved by simply performing degree elevation and surface reconstruction using Bernstein-Bézier tetrahedra of polynomial degree $p=3$ rather than $p=6$.

Finally, Figure 25 shows a mixed-element boundary layer mesh around an aircraft wing, composed of cubic Bernstein-Bézier elements. In this example, a boundary layer mesh of hexahedra is created around the wing surface, but transitions to tetrahedra via a layer of pyramids in the far field. Of course, a boundary layer mesh would normally consist of many, much thinner, hexahedral elements, but we just show a single, thicker, layer here for illustration purposes. For this mesh we begin with a NURBS representation of the aircraft wing shown in Figure 25a. Then, the hexahedral and pyramidal boundary layer elements were created by our own simple boundary layer mesh tool (Figure 25b). Triangle [35] was 
then used to create a quality linear surface mesh of the bounding box, and Tetgen was then used to create the linear volumetric mesh (Figure 25c). Figure 25d shows a detailed view of the boundary layer mesh transitioning to the tetrahedral mesh.

These three examples highlight several key aspects of our meshing procedure. First, the detailed views shown in Figure 23c and Figure 24c illustrate an important property of unstructured surface meshes. Namely, the length scale of the surface mesh is constrained by the length scale of the local Bézier elements as a result of the criteria imposed on the surface mesh outlined in Section 6.1. Second, the bike frame and the propeller serve to show how our method can easily generate either geometrically exact meshes or lower-order approximations. It is also worth noting that although the bike mesh is not geometrically exact, the lower-order mesh approximates the exact geometry with a great deal of accuracy. Finally, the boundary mesh around the airfoil illustrates one of the attractive advantages of mixed-element meshes, namely the ability to create geometrically exact volumetric meshes at relatively low polynomial degree.

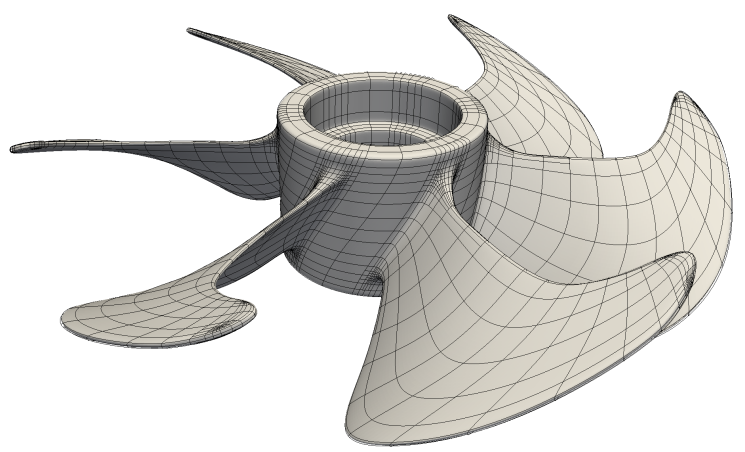

(a)

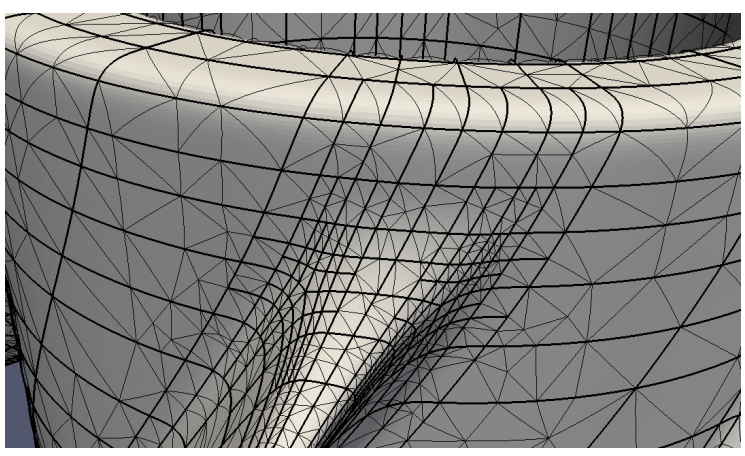

(c)

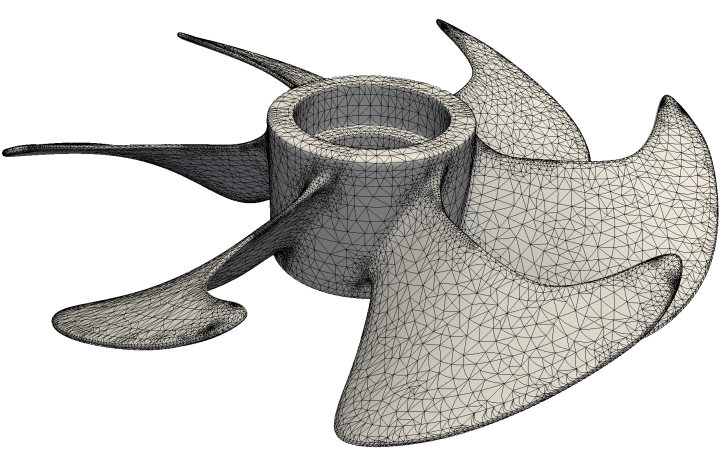

(b)

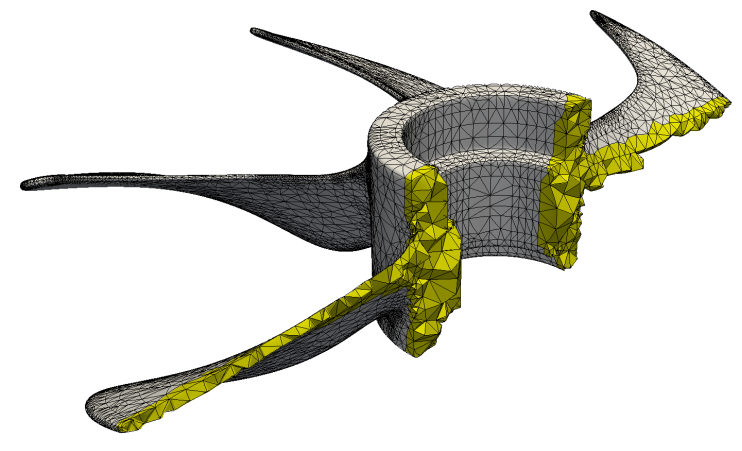

(d)

Figure 23: Geometrically exact mesh of a marine propeller using sextic Bézier tetrahedra. (a) T-spline surface. (b) Surface mesh. (c) Surface mesh detail. (d) Cut view of interior mesh. 


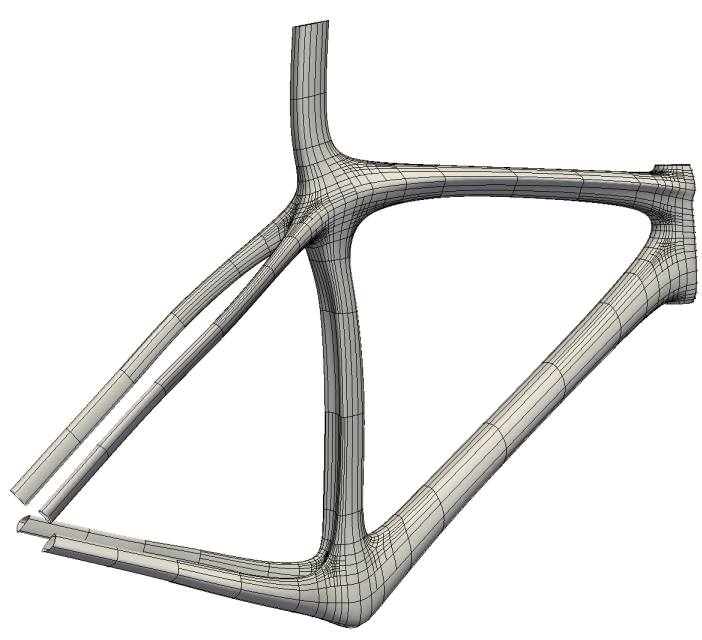

(a)

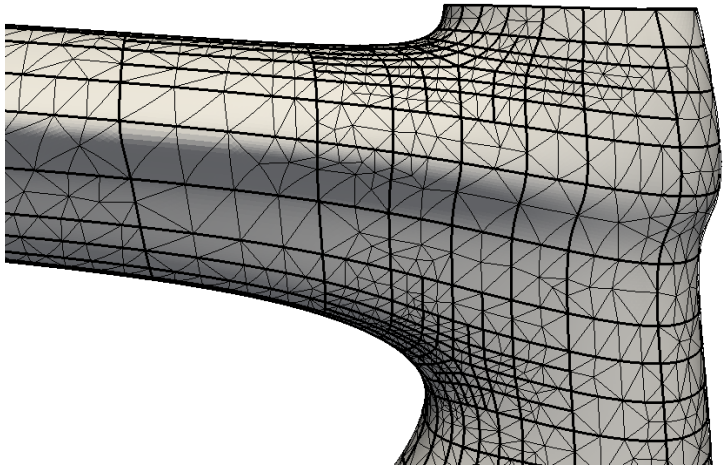

(c)

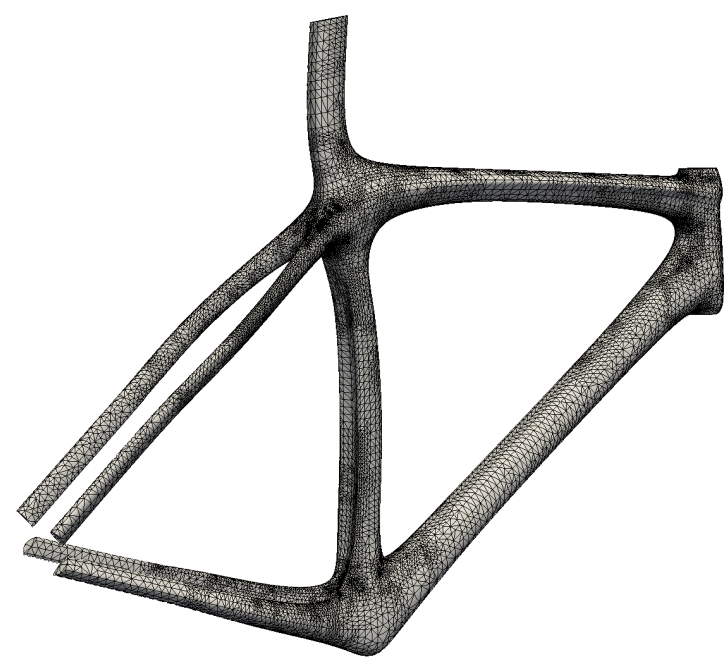

(b)

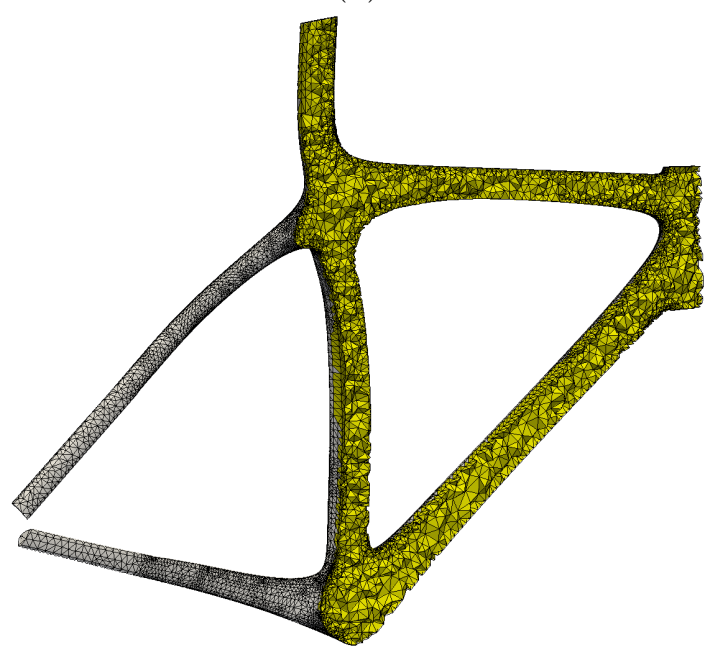

(d)

Figure 24: Lower-order mesh of a bike frame using cubic Bézier tetrahedra. (a) T-spline surface. (b) Surface mesh. (c) Surface mesh detail. (d) Cut view of interior mesh. 


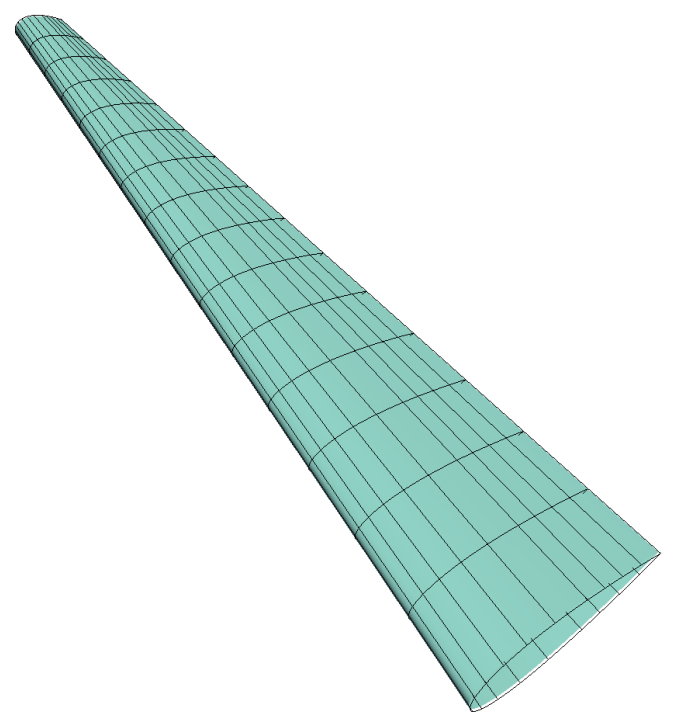

(a)

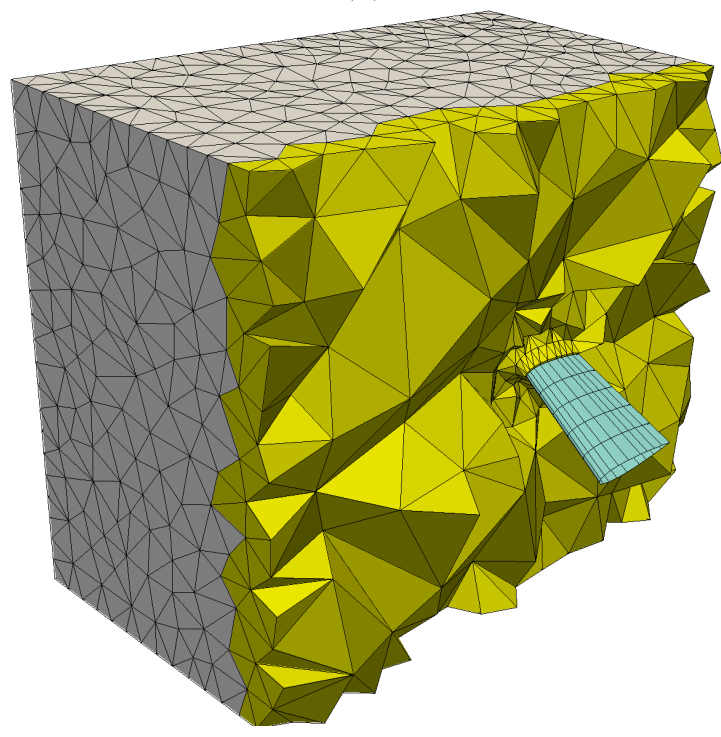

(c)

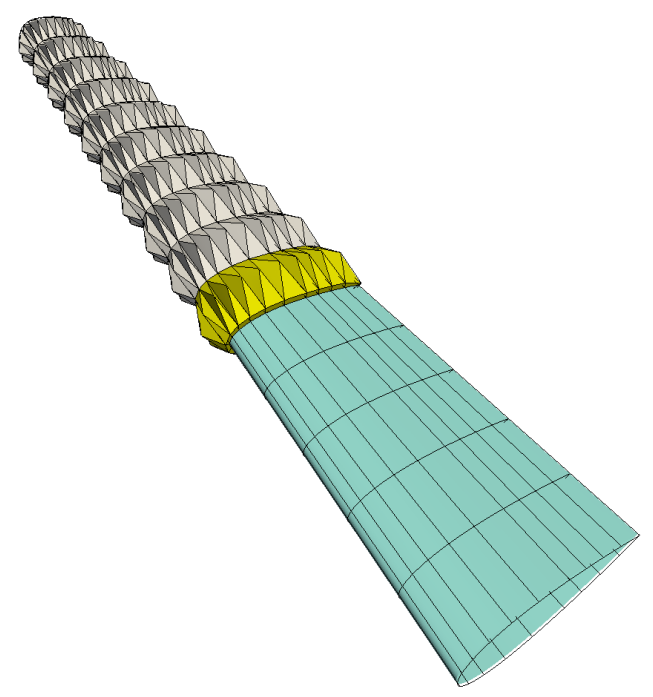

(b)

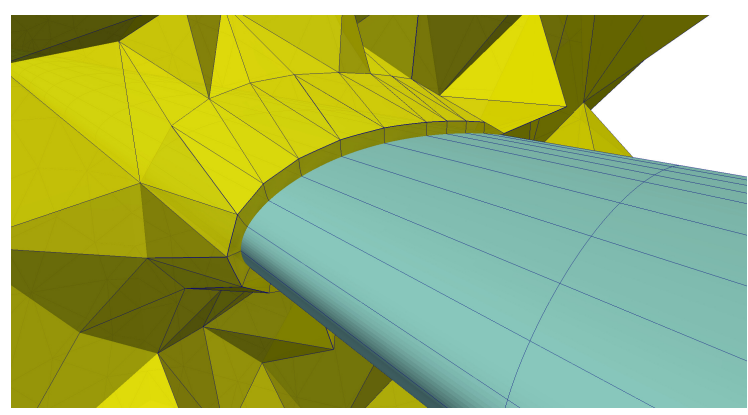

(d)

Figure 25: Mesh of the volume around an aircraft wing. (a) NURBS surface of the aircraft wing. (b) Boundary layer mesh of hexahedra and pyramids. (c) Cut view of volume mesh. (d) Volume mesh detail. 


\section{$9 \quad$ Mesh Quality}

As discussed in Section 6.4, care must be taken to ensure that our method produces quality meshes, with the clear motivation being the desire to create analysis suitable meshes for use with the finite element method. In this section, we present two metrics for evaluating the quality of higher order meshes. We then demonstrate by example how poor quality elements can arise in the mesh generation procedure, and conclude by highlighting some important mesh quality considerations for higher-order mesh generation.

First and foremost, to generate a quality mesh, the original linear elements must be of acceptable quality with regards to traditional mesh quality metrics such as aspect ratio and skewness. However, while ensuring linear mesh quality is necessary for ensuring quality of the degree elevated mesh, it by no means sufficient. Depending on the mesh in question, the surface reconstruction step can introduce undesirable mesh distortion. For example, Figure 26a shows a good quality linear tetrahedron. This tetrahedron is then degree elevated by linear interpolation to a cubic Bézier tetrahedron (shown in Figure 26b), and at this point still has good quality. However, Figures 26c-e show several highly distorted tetrahedra obtained through different edge and face replacements. We desire then to define some easily computable metrics to quantify element quality for higher order elements.

To define the first metric, first recall that for any isoparametric element, we have a bijective mapping of the form $\vec{x}^{E}: \hat{\Omega} \rightarrow \Omega^{E}$, for which we can define a Jacobian of the form $\nabla \vec{x}^{E}$. We can then define our first element quality metric as:

$$
J^{E}=\frac{\inf _{\vec{\xi} \in \Omega^{E}}\left|\operatorname{det}\left(\nabla \vec{x}^{E}\right)\right|}{\sup _{\vec{\xi} \in \Omega^{E}}\left|\operatorname{det}\left(\nabla \vec{x}^{E}\right)\right|}
$$

Intuitively speaking, the metric $J^{E}$ is a measure of the variation in the magnitude of the mapping $\vec{x}^{E}$ across the element. This can be likened to a higher-order notion of mesh regularity. The range of $J^{E}$ is $J^{E} \in[0,1]$, with element quality increasing as $J^{E} \rightarrow 1$.

To define the second metric, let us recall the mapping $\vec{x}^{\text {poly,E}}: \hat{\Omega} \rightarrow \Omega^{\text {poly,E}}$ that maps the reference polyhedron $\hat{\Omega}$ to the linear polyhedron in physical space $\Omega^{\text {poly,E}}$. We can then define a composite mapping $\vec{x}_{s}^{E}=\vec{x}^{E} \circ\left(\vec{x}^{p o l y, E}\right)^{-1}$ which maps the linear polyhedron $\Omega^{\text {poly }, E}$ to the curvilinear polyhedron $\Omega^{E}$. The mapping $\vec{x}_{s}^{E}$ encodes the nonlinear nature of the mapping $\vec{x}^{E}$. Now, let $\sigma_{i}$ denote the singular values of the singular value decomposition of $\nabla \vec{x}_{s}^{E}$. We then define our second quality metric as:

$$
S^{E}=\frac{\inf _{\vec{\xi} \in \Omega^{E}} \min \left(\sigma_{i}\right)}{\sup _{\vec{\xi} \in \Omega^{E}} \max \left(\sigma_{i}\right)}
$$

The metric $S^{E}$ can be thought of as a generalization of the element skewness to higher-order elements. The range of $S^{E}$ is $S^{E} \in[0,1]$, with element quality increasing as $S^{E} \rightarrow 1$. We note a slight divergence here from the classical definition of skewness, which is also defined on $[0,1]$, but with element quality increasing as $S \rightarrow 0$.

Table 2 shows the values of $J^{E}$ and $S^{E}$ for the elements shown in Figure 26b-e. It should be noted that rather than taking a true infimum or supremum as noted in the definitions of $J^{E}$ and $S^{E}$, we instead take a sampling over quadrature points. In particular, we sample a collapsed $(p+1)^{d_{s}}$-point Gaussian quadrature rule. The tetrahedron in $26 \mathrm{~b}$ is an ideal 
higher order tetrahedron, with $J^{E}=S^{E}=1$, but the quality degrades considerably under the distortions shown in Figures 26c-e. We note that these quality metrics are completely independent of the linear quality of the element, and only measure the higher order distortion of the element. For example, Figure 26f shows the results of degree elevating a poor quality linear "spire" tetrahedron. Despite the poor linear quality, $J^{E}=S^{E}=1$, since the higher order nodes are equispaced. Lastly, we note that while these distortions are illustrated only on a tetrahedra, the concept extends readily to hexahedra, pyramids and wedges.

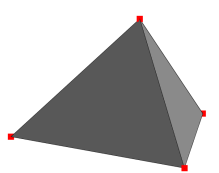

(a)

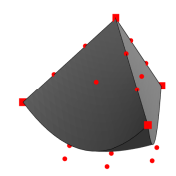

(d)

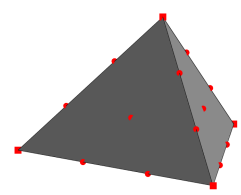

(b)

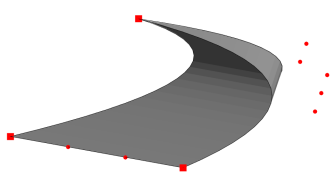

(e)

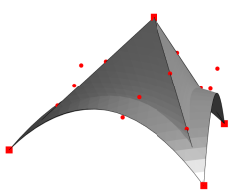

(c)

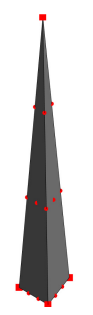

(f)

Figure 26: (a) An equilateral linear tetrahedron. (b) An equilateral cubic tetrahedron. (c-e) Various highly skewed tetrahedra. (f) A poor linear quality, good higher-order quality tetrahedron.

Table 2: Mesh quality for the tetrahedra in Figure 26 as sampled at quadrature points.

\begin{tabular}{|l|c|c|}
\hline & $J^{E}$ & $S^{E}$ \\
\hline $\mathrm{b}$ & 1 & 1 \\
\hline $\mathrm{c}$ & 0.019 & 0.0067 \\
\hline $\mathrm{d}$ & 0.4239 & 0.2302 \\
\hline $\mathrm{e}$ & 1 & 0.1930 \\
\hline $\mathrm{f}$ & 1 & 1 \\
\hline
\end{tabular}

With element-wise quality metrics established, we can use these metrics to define some notions of global mesh quality, viz.:

$$
J=\min _{\Omega^{E} \in \Omega_{M}} J^{E}
$$




$$
\begin{aligned}
& \bar{J}=\operatorname{mean}_{\Omega^{E} \in \Omega_{M}} J^{E} \\
& S=\min _{\Omega^{E} \in \Omega_{M}} S^{E} \\
& \bar{S}=\operatorname{mean}_{\Omega^{E} \in \Omega_{M}} S^{E}
\end{aligned}
$$

To further motivate the need for higher-order mesh quality metrics, we present a practical example of how mesh quality can degrade under surface reconstruction. Consider the linear element shown in Figure 29a. Under surface reconstruction, the corresponding higher order element becomes poorly conditioned, with the dihedral angle between the two boundary faces of the tetrahedra approaching $\theta=180^{\circ}$, as shown in Figure 29b. If the original linear element is split, the resulting higher order elements have much better quality, as shown in Figure 29c. Additionally, we have shown in previous work that poor element quality does not necessarily preclude optimal convergence, but we must guarantee that $J \rightarrow 1$ and $S \rightarrow 1$ under mesh refinement $[10,27]$. However, the Jacobian of the mapping $\vec{x}^{e}$ for the element shown in Figure 29b is singular where the dihedral angle approaches $\theta=180^{\circ}$. As such, the quality metrics for any mesh containing such an element will not approach unity under refinement. Figure 27 shows how the metrics $S$ and $J$ change under refinement for (a) the cylindrical mesh containing the bad quality element, and (b) the cylindrical mesh with the bad element removed by splitting.

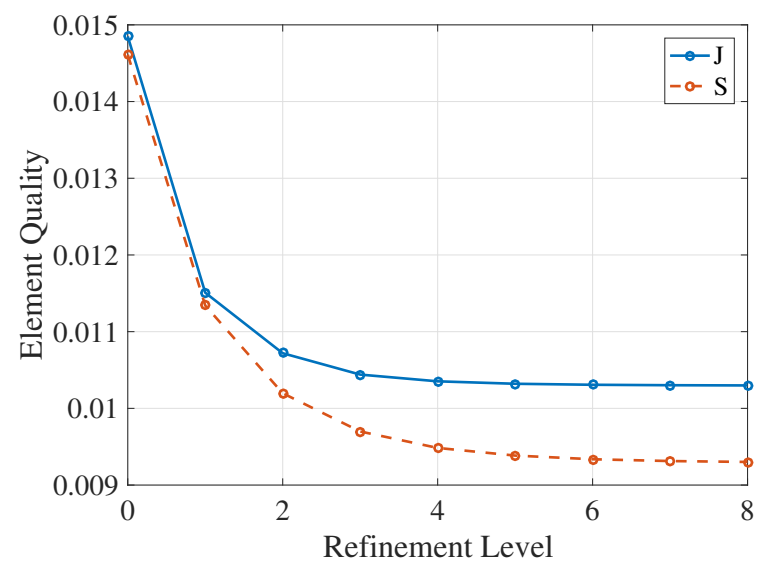

(a)

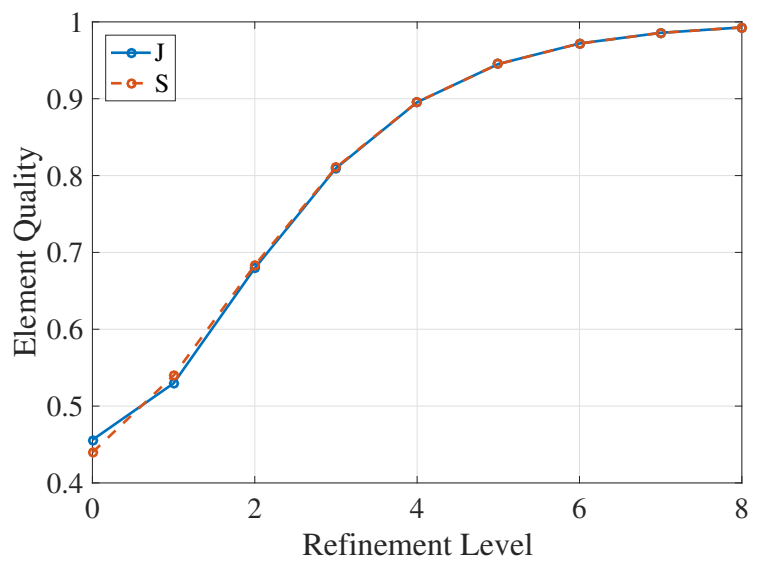

(b)

Figure 27: Mesh quality metrics under mesh refinement as sampled at quadrature points for: (a) the mesh of a cylinder with a bad element, and (b) the mesh of a cylinder with the bad element removed.

Finally, we demonstrate the ability of our mesh generation procedure to produce quality meshes for complex geometries of engineering interest. Figure 28 provides histograms of mesh element quality for the mesh of the bike frame shown in Figure 24. From the histograms, we see that the splitting and smoothing process dramatically improves the mesh quality on a global scale. We do note that there remain a very small number of poor quality 
elements, namely 6 elements with $J^{E}<0.05$ and 23 elements with $S^{E}<0.05$. This is a consequence of the fact that we seek a higher-order, geometrically exact mesh with a minimal number of total elements. Regardless, we have obtained high quality results using the bike frame mesh as demonstrated in Section 10.3. We are currently establishing a complete theory of mesh quality for meshes composed of Bernstein-Bézier elements, and are in the process of developing a meshing procedure capable of generating meshes that satisfy user-defined quality constraints (i.e. $J \leq c_{1}$ and $S \leq c_{2}$, where $c_{1}$ and $c_{2}$ are user-provided constants).

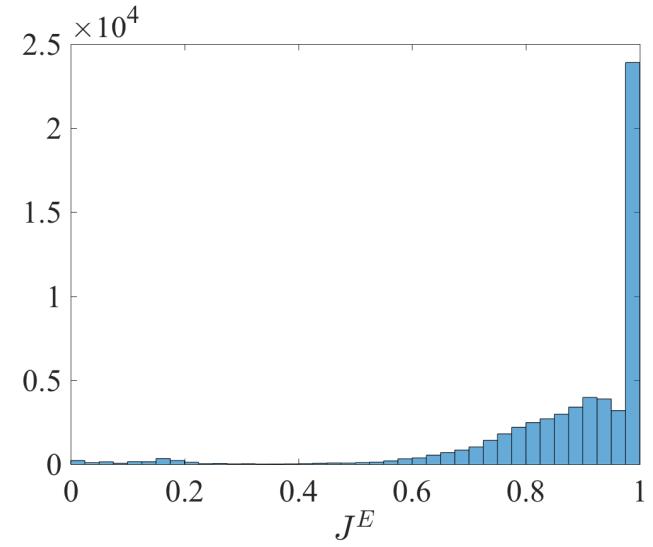

(a)

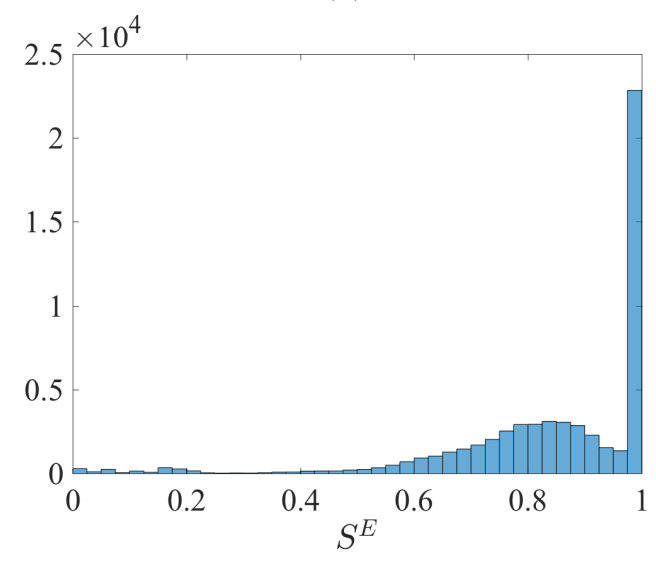

(c)

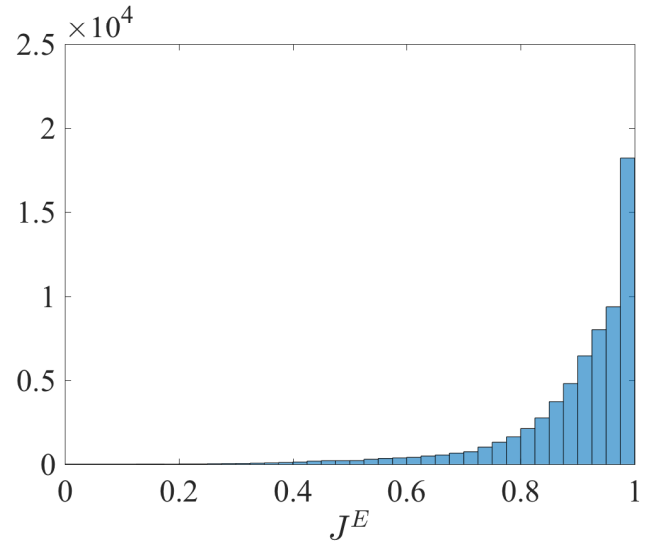

(b)

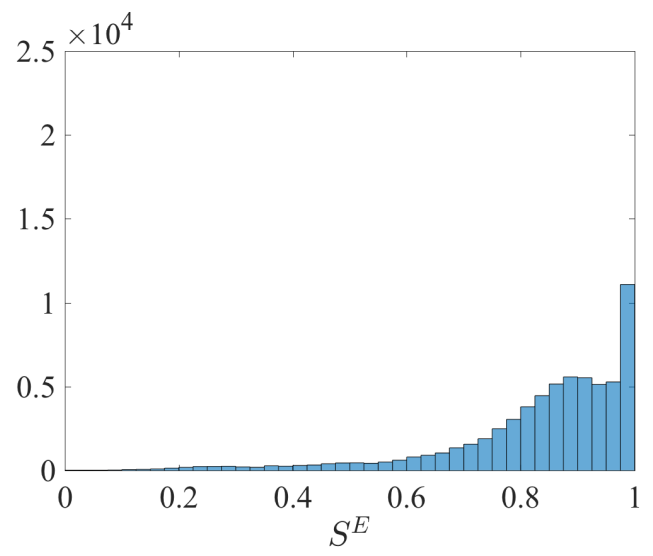

(d)

Figure 28: Distribution of element quality for mesh of the bike frame. (a) $J^{E}$ before splitting and smoothing. (b) $J^{E}$ after splitting and smoothing. (c) $S^{E}$ before splitting and smoothing. (d) $S^{E}$ after splitting and smoothing.

While we do not present any methods for automated mesh optimization in this paper, we have considered element quality here at length for several reasons. First, this section highlights the fact that care must be taken in the mesh generation process, particularly during linear mesh generation and surface reconstruction. Additionally, meshes produced by the mesh generation procedure outlined in this paper should be evaluated using these 
metrics. In cases of poor mesh quality, steps should be taken to improve mesh quality, either by improving the linear mesh, or through mesh smoothing. Finally, this section provides motivation for future work towards developing algorithms for automatic higher order mesh optimization, and quality mesh generation.

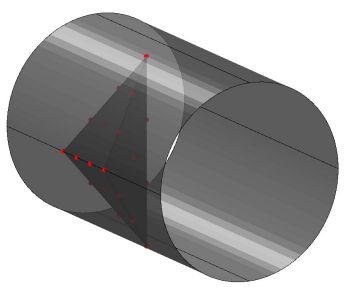

(a)

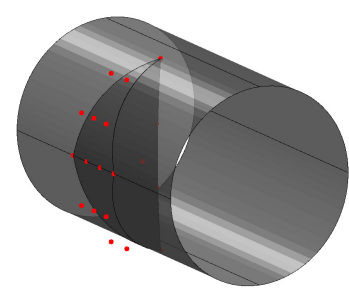

(b)

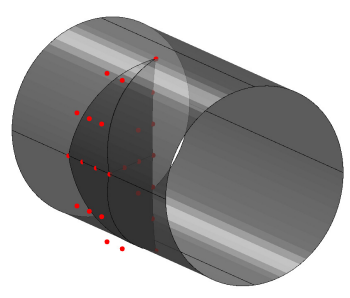

(c)

Figure 29: Example of a poor quality tetrahedron generated during surface reconstruction. (a) The original linear tetrahedron. (b) The poor quality higher order tetrahedron generated by surface reconstruction. (c) Improving element quality by splitting the poor quality element in two.

\section{Numerical Examples}

We now turn our attention from the problem of mesh generation to that of the analysis suitability of these discretizations. Specifically, we perform three numerical tests. First, a patch test is performed to demonstrate that the elements presented here are capable of exactly representing linear solutions, even under large deformation. Second, the method of manufactured solutions is used to verify that optimal convergence rates are observed. Finally, we present the solution of a linear elasticity problem over the bike frame from Figure 24 to demonstrate the analysis suitability of a more complicated geometry. For brevity, we consider only cubic Bernstein-Bézier discretizations in what follows.

\subsection{Patch Test}

Following Lipton et al. [21], we would like to show that these proposed higher order elements are capable of exactly representing certain solutions, even under severe mesh distortion. Since the behavior of hexahedra have already been extensively studied in the previous literature, we will limit our tests to tetrahedra, pyramids and wedges. For each class of element, we create a discretization of the unit cube using only that element type. in each case, the mesh is created so that each element has at least one face on the global boundary of the cube, and all the elements share a single vertex in the center. Figure 30 shows the meshes for each of the three geometric primitives. Then for each mesh, we perform two patch tests to study the behavior of the elements under severe distortion.

Test 1: In the first test, the center node that is shared by all elements is moved to one of the corners of the cube, causing the mesh to become skewed as is shown in Figure 31. 


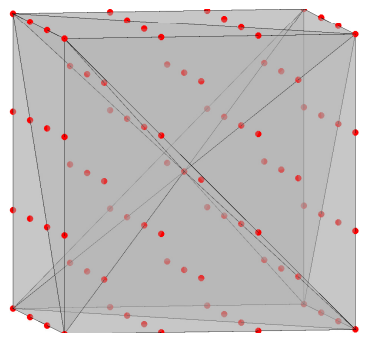

(a)

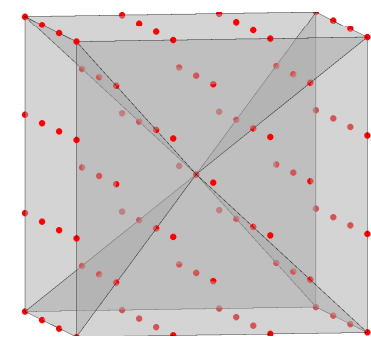

(b)

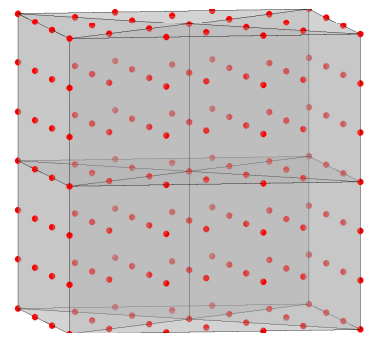

(c)

Figure 30: Undeformed meshes for the patch test. (a)Tetrahedral mesh. (b) Pyramidal mesh. (c) Wedge mesh.

However, the distribution of internal nodes still remains linear across the element. That is, the linear quality depreciates significantly, but $J^{E}=S^{E}=1$.

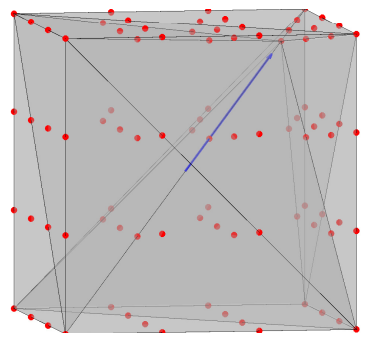

(a)

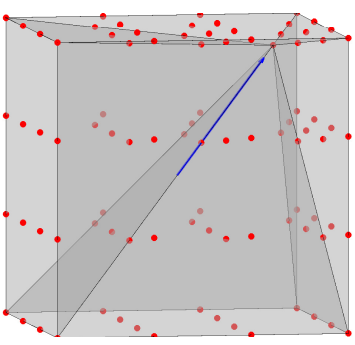

(b)

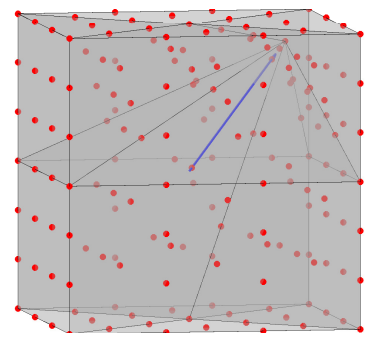

(c)

Figure 31: Deformations for the first patch test. (a)Tetrahedral mesh. (b) Pyramidal mesh. (c) Wedge mesh.

Test 2: In the second test, the center node is left in the center, but internal nodes of the Bézier elements are collapsed to the center point as shown in Figure 32. In this way, the linear quality remains unchanged, but $J^{E} \rightarrow 0$ and $S^{E} \rightarrow 0$.

We then solve two linear elasticity problems over each mesh, a constant tension, and constant shear Dirichlet problem, as illustrated in Figure 33. In each case, the bottom nodes are held fixed. In the constant tension case, we apply a uniform displacement of $d=0.1$ in the $z$ direction to the top nodes. In the constant shear case, we apply a uniform displacement of $d=0.1$ in both the $x$ and $y$ direction to the top nodes. We also enforce zero $z$ displacement on the sides of the cube. For each test, we gradually increase the mesh deformation from 0 (no deformation) to 1 (fully deformed), and evaluate the solution for each patch test at the control points on each element. If the computed solution agrees with the theoretical solution to within 13 decimal places for both the constant tension and constant shear case, we say that the patch test is passed. Table 3 shows the maximum deformation achieved for each test and element type before the patch test was no longer passed. 


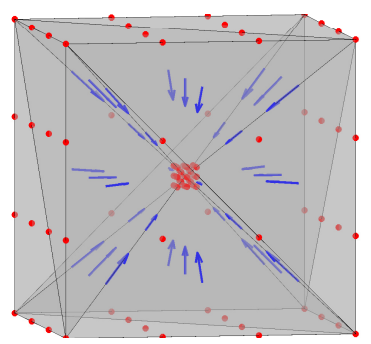

(a)

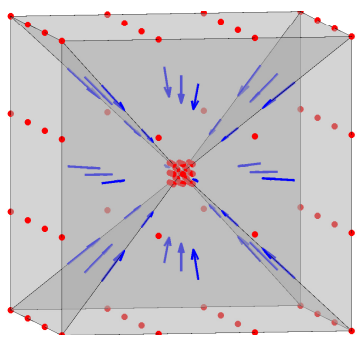

(b)

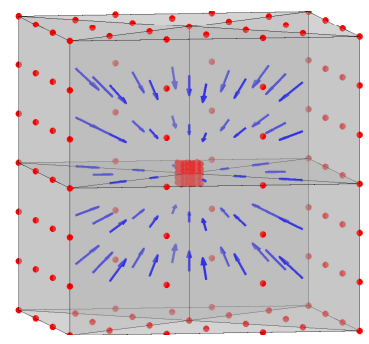

(c)

Figure 32: Deformations for the second patch test. (a)Tetrahedral mesh. (b) Pyramidal mesh. (c) Wedge mesh.

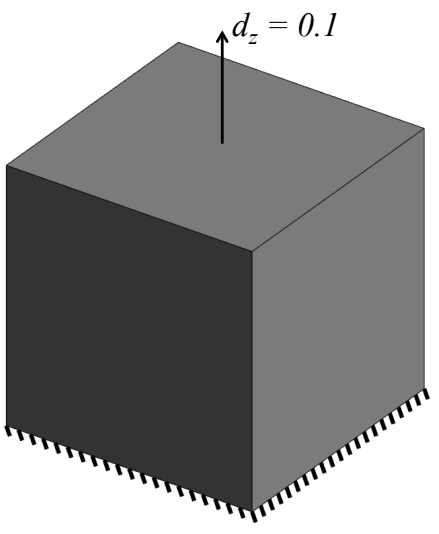

(a)

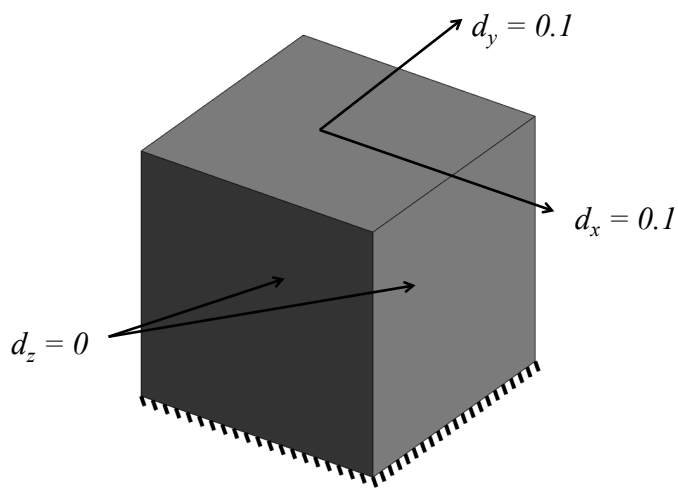

(b)

Figure 33: Boundary conditions for (a) the constant tension, and (b) the constant shear patch tests.

Table 3: Patch Test Results

\begin{tabular}{|l|c|c|}
\hline & Test 1 & Test 2 \\
\hline Tetrahedra & 0.99 & 0.99 \\
\hline Pyramids & 0.98 & 0.99 \\
\hline Wedges & 0.5 & 0.99 \\
\hline
\end{tabular}




\subsection{Method of Manufactured Solutions}

We also demonstrate higher order convergence of our method using the method of manufactured solutions. For the test, we consider three different meshes of a cylinder, including (a) a pure tetrahedral mesh, (b) a pure wedge mesh and (c) a mixed element mesh consisting of hexahedra, pyramids, and and tetrahedra. The three meshes are shown below in Figure 34. For each mesh, we define a temperature field over the cylinder defined by the function:

$$
\phi(x, y, z)=(x / L)^{2} y^{2} z^{2}
$$

The temperature field is shown in Figure 34d. We then enforce Dirichlet boundary conditions on the faces of the cylinder, and solve Poisson's equation for the temperature field over the domain under successive levels of refinement. The convergence plots of the error in the $L_{2}$ and $H_{1}$ norms versus the non-dimensional mesh size for each mesh are shown in Figure 35. As is readily seen from Figures 35a-b, the tetrahedral meshes exhibit optimal convergence rates after several levels of refinement. Additionally, both the wedge mesh and the mixed element mesh approach optimal convergence rates under successive refinement, as can be seen in Figures 35c-d and Figures 35e-f, respectively.

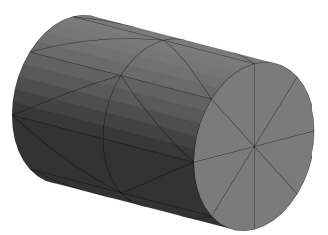

(a)

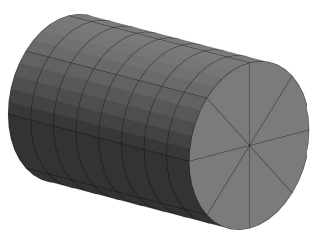

(b)

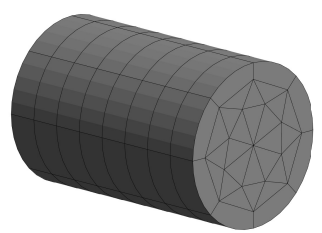

(c)

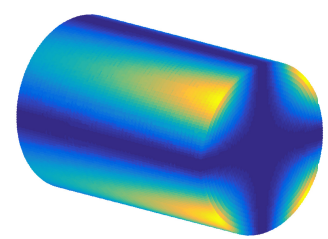

(d)

Figure 34: Meshes used with the method of manufactured solutions. (a) Tetrahedral mesh (b) Wedge mesh. (c) Mixed element hexahedral-pyramidal-tetrahedral mesh. (d) The prescribed temperature field.

\subsection{Practical Examples}

Finally, we present some analysis results for some slightly more interesting geometries. First, we simulate the effect of torsion on the propeller from Figure 23 by solving a linear elasticity problem using a superparametric approach. We prescribe zero displacement boundary conditions on the interior radius of the propeller, and prescribe set displacements for the propeller tips, as shown in Figure 36a. We then solve the linear elasticity equations over the mesh of sextic tetrahedra, while using cubic Bernstein polynomials as the basis for analysis. Figure 36b shows the resulting displacement magnitude.

Next, we solve a linear elasticity problem over the bike frame from Figure 24. The head tube and rear stays are held fixed as shown in Figure 37a, and a downward force is applied on the top of the seat post. For the bike simulation, we employed an isoparametric approach, using cubic Bernstein polynomials as the basis for a simulation over the cubic tetrahedral mesh. Figure 37b illustrates the magnitude of the displacement of the bike under the applied load. For both examples, the results seem to be accurate, at least from an intuitive sense, 


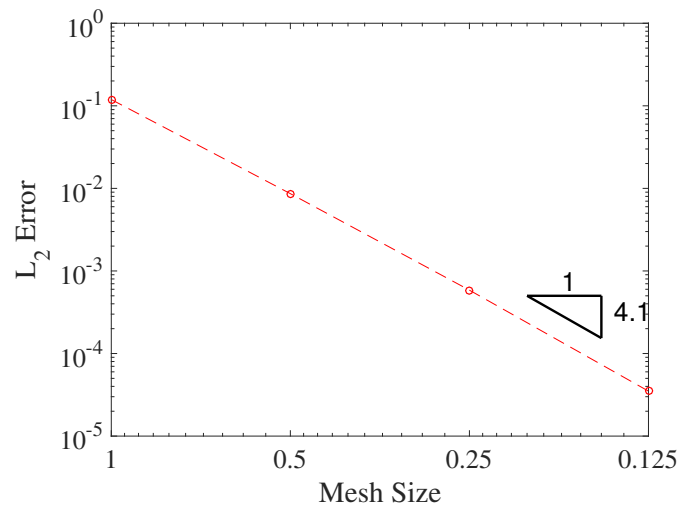

(a)

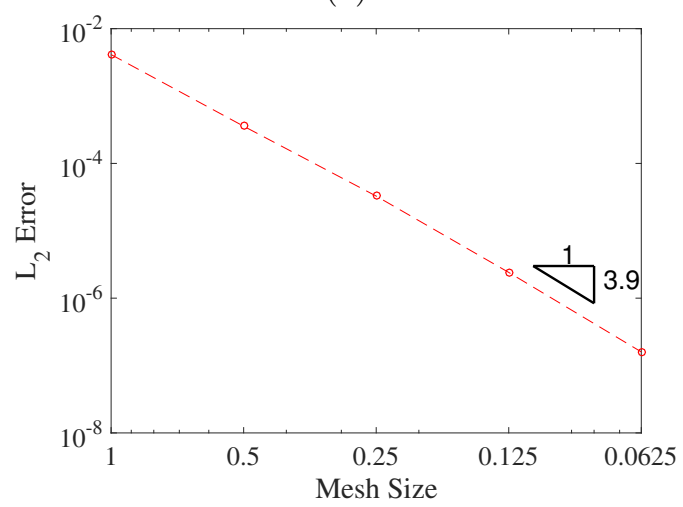

(c)

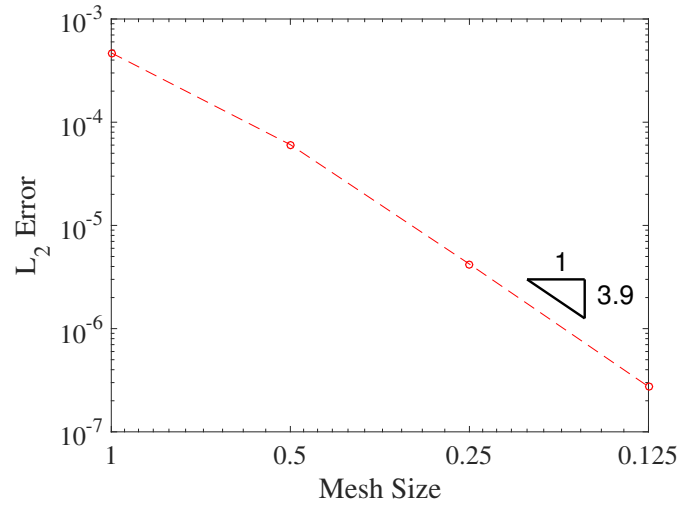

(e)

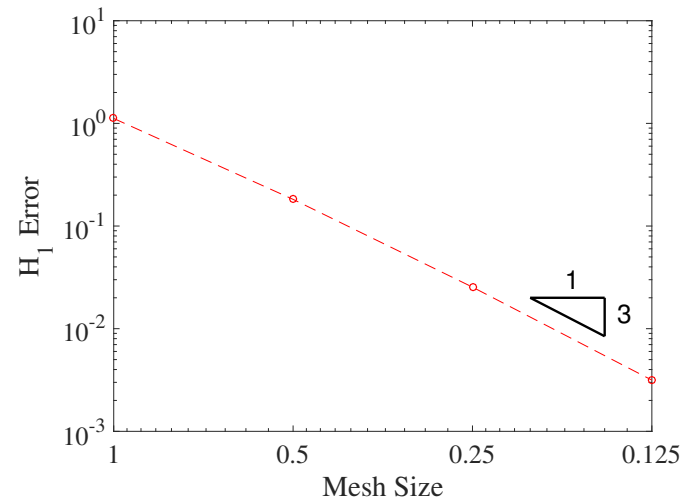

(b)

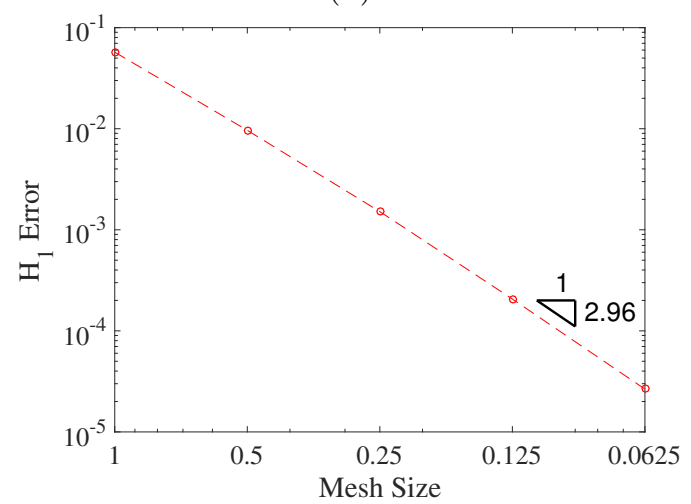

(d)

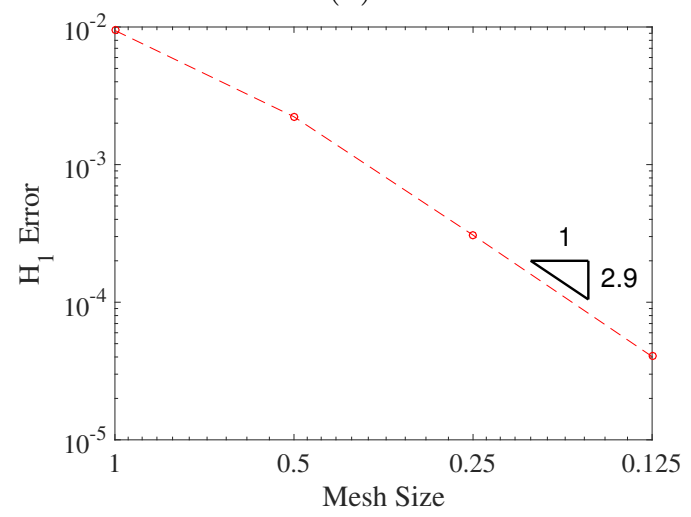

(f)

Figure 35: Convergence plots for three different meshes (a,b) $L_{2}$ and $H_{1}$ convergence plots for the tetrahedral mesh. (c,d) $L_{2}$ and $H_{1}$ convergence plots for the wedge mesh. (e,f) $L_{2}$ and $H_{1}$ convergence plots for the mixed element mesh. 
and, coupled with the other numerical tests in this section, give us confidence in the analysis suitability of these discretizations.

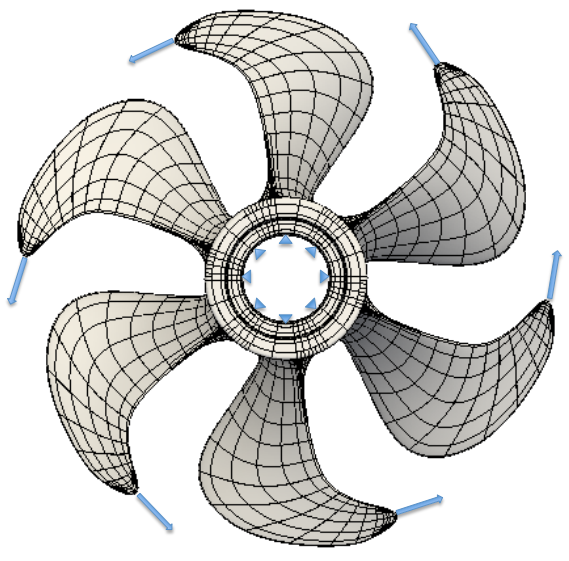

(a)

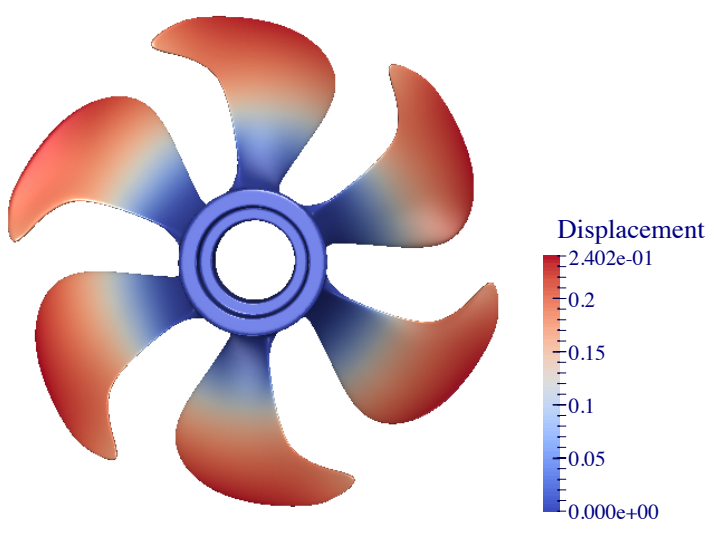

(b)

Figure 36: Superparametric simulation of linear elasticity on the propeller. (a) Boundary conditions. (b) Displacement magnitude.

\section{Conclusions}

In this paper, we have outlined a framework for isogeometric analysis using unstructured mixed-element discretizations. We first presented our method for creating geometrically exact meshes composed of rational Bézier elements through degree elevation of suitable linear meshes. Additionally, we introduced the concept of $P_{2}$ complexity, and noted that to guarantee a mesh is geometrically exact, we are often required to use elements of high polynomial degree ( $p=6$ for a bicubic NURBS bounding surface). We further demonstrated by example that our framework allows for the construction of geometrically exact meshes for superparameteric finite element analysis as well as the construction of approximate lowerorder meshes for isoparameteric finite element analysis. Alternatively, we demonstrated that cubic pyramidal Bézier elements may be utilized to connect a bicubic surface to a mesh of cubic tetrahedral Bézer elements in a watertight and geometrically exact fashion. Finally, we discussed some important mesh quality considerations, and concluded with a demonstration of the analysis suitability of unstructured Bernstein-Bézier discretizations. While the method outlined here is a promising step towards solving the problem of surface-to-volume parameterization, we note that there are several avenues of future research that should be considered. Most importantly, we note further work on the problem of mesh optimization is required. Although we outline the requirements for ensuring optimal convergence rates under mesh refinement, an automated mesh optimization algorithm would be invaluable for the automatic generation of quality higher-order meshes for analysis. Additionally, we have largely ignored the problem of trimming curves in the current work, as the goal was focus on geometrically exact mesh generation. However, we note that Xia and Qian presented a method for handling trimming curves in their recent work [41]. Their approach is able 


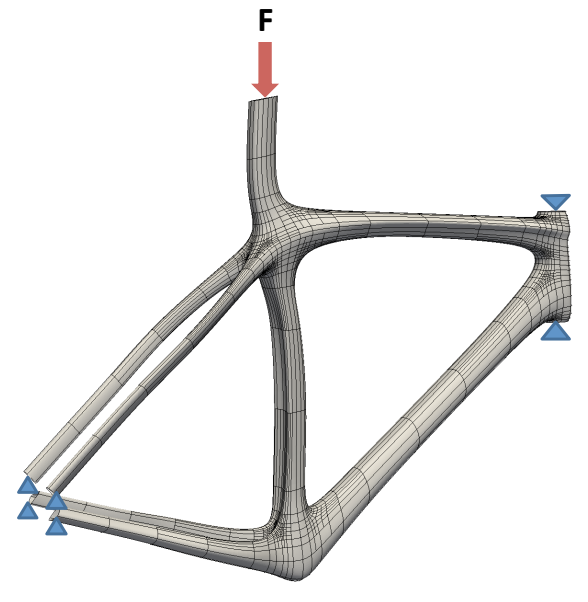

(a)

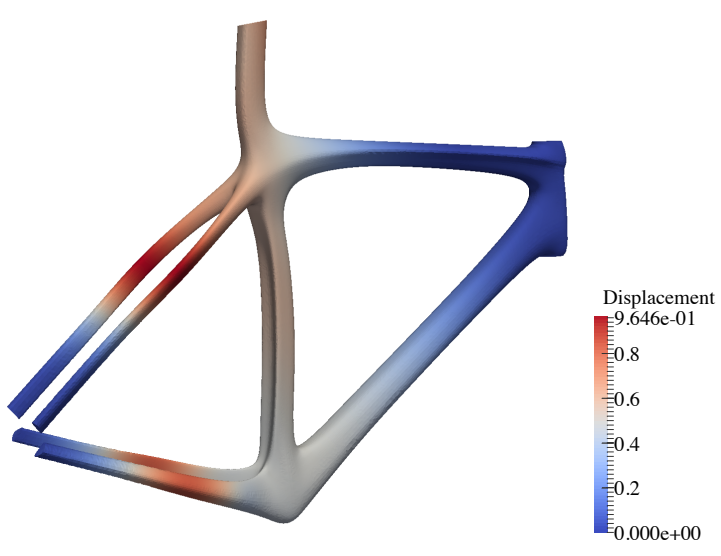

(b)

Figure 37: Lower-order isoparametric simulation of linear elasticity on a bike frame. (a) Boundary conditions. (b) Displacement magnitude.

to generate meshes that are geometrically exact with the exception of the elements that border trimming curves. In future work, we plan to employ a similar approach to extend our meshing capabilities to include meshing trimmed objects.

\section{Acknowledgements}

This material is based upon work supported by the National Science Foundation Graduate Research Fellowship Program under Grant No. DGE 1144083 as well as work supported by the Air Force Office of Scientific Research under Grant No. FA9550-14-1-0113. The authors would also like to thank the reviewers, whose suggestions greatly improved the quality of this paper.

\section{References}

[1] M. Aigner, C. Heinrich, B. Jüttler, E. Pilgerstorfer, B. Simeon, and A.-V. Vuong. Swept Volume Parameterization for Isogeometric Analysis. In Edwin R. Hancock, Ralph R. Martin, and Malcolm A. Sabin, editors, Mathematics of Surfaces XIII, number 5654 in Lecture Notes in Computer Science, pages 19-44. Springer Berlin Heidelberg, 2009.

[2] M. Ainsworth, O. Davydov, and L. L. Schumaker. Bernstein-Bézier finite elements on tetrahedral-hexahedral-pyramidal partitions. Computer Methods in Applied Mechanics and Engineering, 304:140-170, June 2016.

[3] H. Al Akhras, T. Elguedj, A. Gravouil, and M. Rochette. Isogeometric analysis-suitable trivariate NURBS models from standard B-Rep models. Computer Methods in Applied Mechanics and Engineering, 307:256-274, August 2016. 
[4] Y. Bazilevs, V. M. Calo, J. A. Cottrell, J. A. Evans, T. J. R. Hughes, S. Lipton, M. A. Scott, and T. W. Sederberg. Isogeometric analysis using T-splines. Computer Methods in Applied Mechanics and Engineering, 199(5-8):229-263, January 2010.

[5] P. E. Bernard, J. F. Remacle, N. Kowalski, and C. Geuzaine. Frame field smoothnessbased approach for hex-dominant meshing. Computer-Aided Design, 72:78-86, March 2016 .

[6] M. J. Borden, M. A. Scott, J. A. Evans, and T. J. R. Hughes. Isogeometric finite element data structures based on Bézier extraction of NURBS. International Journal for Numerical Methods in Engineering, 87(1-5):15-47, July 2011.

[7] J. Chan and T. Warburton. A short note on a Bernstein-Bezier basis for the pyramid. arXiv:1508.05609 [math], August 2015. arXiv: 1508.05609.

[8] L. P. Chew. Guaranteed-quality Mesh Generation for Curved Surfaces. In Proceedings of the Ninth Annual Symposium on Computational Geometry, SCG '93, pages 274-280, New York, NY, USA, 1993. ACM.

[9] J. J Chou. Higher order Bézier circles. Computer-Aided Design, 27(4):303-309, April 1995.

[10] L. Engvall and J. A. Evans. Isogeometric triangular Bernstein-Bézier discretizations: Automatic mesh generation and geometrically exact finite element analysis. Computer Methods in Applied Mechanics and Engineering, 304:378-407, June 2016.

[11] J. M. Escobar, J. M. Cascón, E. Rodríguez, and R. Montenegro. A new approach to solid modeling with trivariate T-splines based on mesh optimization. Computer Methods in Applied Mechanics and Engineering, 200(45-46):3210-3222, October 2011.

[12] J. M. Escobar, R. Montenegro, E. Rodríguez, and J. M. Cascón. The meccano method for isogeometric solid modeling and applications. Engineering with Computers, 30(3):331-343, December 2012.

[13] P.l. George and H. Borouchaki. Construction of tetrahedral meshes of degree two. International Journal for Numerical Methods in Engineering, 90(9):1156-1182, June 2012 .

[14] C. Geuzaine and J.-F. Remacle. Gmsh: A 3-D finite element mesh generator with builtin pre- and post-processing facilities. International Journal for Numerical Methods in Engineering, 79(11):1309-1331, September 2009.

[15] A. Haselbacher and J. Blazek. Accurate and Efficient Discretization of Navier-Stokes Equations on Mixed Grids. AIAA Journal, 38(11):2094-2102, 2000.

[16] T. J. R. Hughes, J. A. Cottrell, and Y. Bazilevs. Isogeometric analysis: CAD, finite elements, NURBS, exact geometry and mesh refinement. Computer Methods in Applied Mechanics and Engineering, 194(39-41):4135-4195, October 2005. 
[17] A. Johnen, J. F. Remacle, and C. Geuzaine. Geometrical validity of curvilinear finite elements. Journal of Computational Physics, 233:359-372, January 2013.

[18] M.-J. Lai and L. L. Schumaker. Spline Functions on Triangulations. Cambridge University Press, April 2007. Google-Books-ID: 6hvqGgbBmEoC.

[19] T. S. Lan and S. H. Lo. Finite element mesh generation over analytical curved surfaces. Computers \& Structures, 59(2):301-309, April 1996.

[20] X. Li, J. Zheng, T. W. Sederberg, T. J. R. Hughes, and M. A. Scott. On linear independence of T-spline blending functions. Computer Aided Geometric Design, 29(1):63-76, January 2012.

[21] S. Lipton, J. A. Evans, Y. Bazilevs, T. Elguedj, and T. J. R. Hughes. Robustness of isogeometric structural discretizations under severe mesh distortion. Computer Methods in Applied Mechanics and Engineering, 199(5-8):357-373, January 2010.

[22] L. Liu, Y. Zhang, Thomas J. R. Hughes, M. A. Scott, and T. W. Sederberg. Volumetric T-spline construction using Boolean operations. Engineering with Computers, 30(4):425-439, November 2013.

[23] L. Liu, Y. Zhang, Y. Liu, and W. Wang. Feature-preserving T-mesh construction using skeleton-based polycubes. Computer-Aided Design, 58:162-172, January 2015.

[24] X.J. Luo, M. S. Shephard, R. M. O'Bara, R. Nastasia, and M. W. Beall. Automatic p-version mesh generation for curved domains. Engineering with Computers, 20(3):273285, July 2004.

[25] T. Martin, E. Cohen, and M. Kirby. Volumetric Parameterization and Trivariate Bspline Fitting Using Harmonic Functions. In Proceedings of the 2008 ACM Symposium on Solid and Physical Modeling, SPM '08, pages 269-280, New York, NY, USA, 2008. $\mathrm{ACM}$.

[26] D. J. Mavriplis and V. Venkatakrishnan. A Unified Multigrid Solver for the NavierStokes Equations on Mixed Element Meshes. International Journal of Computational Fluid Dynamics, 8(4):247-263, August 1997.

[27] C. Michoski, J. Chan, L. Engvall, and J. A. Evans. Foundations of the blended isogeometric discontinuous Galerkin (BIDG) method. Computer Methods in Applied Mechanics and Engineering, 305:658-681, June 2016.

[28] P.-O. Persson and J. Peraire. Curved mesh generation and mesh refinement using lagrangian solid mechanics. In Proceedings of the 47th AIAA Aerospace Sciences Meeting and Exhibit, volume 204, 2009.

[29] O. Sahni, K. E. Jansen, M. S. Shephard, C. A. Taylor, and M. W. Beall. Adaptive boundary layer meshing for viscous flow simulations. Engineering with Computers, 24(3):267-285, April 2008. 
[30] M. A. Scott, M. J. Borden, C. V. Verhoosel, T. W. Sederberg, and T. J. R. Hughes. Isogeometric finite element data structures based on Bézier extraction of T-splines. International Journal for Numerical Methods in Engineering, 88(2):126-156, October 2011.

[31] M. A. Scott, X. Li, T. W. Sederberg, and T. J. R. Hughes. Local refinement of analysis-suitable T-splines. Computer Methods in Applied Mechanics and Engineering, 213-216:206-222, March 2012.

[32] T. W. Sederberg, J. Zheng, A. Bakenov, and A. Nasri. T-splines and T-NURCCs. In ACM SIGGRAPH 2003 Papers, SIGGRAPH '03, pages 477-484, New York, NY, USA, 2003. ACM.

[33] M. S. Shephard, J. E. Flaherty, K. E. Jansen, X. Li, X. Luo, N. Chevaugeon, J.F. Remacle, M. W. Beall, and R. M. O'Bara. ADAPT '03: Conference on Adaptive Methods for Partial Differential Equations and Large-Scale ComputationAdaptive mesh generation for curved domains. Applied Numerical Mathematics, 52(2):251-271, February 2005.

[34] S. J. Sherwin and J. Peiró. Mesh generation in curvilinear domains using high-order elements. International Journal for Numerical Methods in Engineering, 53(1):207-223, January 2002.

[35] J.R. Shewchuk. Triangle: Engineering a 2d quality mesh generator and Delaunay triangulator. In Ming C. Lin and Dinesh Manocha, editors, Applied Computational Geometry Towards Geometric Engineering, number 1148 in Lecture Notes in Computer Science, pages 203-222. Springer Berlin Heidelberg, 1996.

[36] H. Si. TetGen, a Delaunay-Based Quality Tetrahedral Mesh Generator. ACM Trans. Math. Softw., 41(2):11:1-11:36, February 2015.

[37] D. Sokolov, N. Ray, L. Untereiner, and B. Lévy. Hexahedral-dominant meshing, October 2015 .

[38] D. C. Thomas, M. A. Scott, J. A. Evans, K. Tew, and E. J. Evans. Bézier projection: A unified approach for local projection and quadrature-free refinement and coarsening of NURBS and T-splines with particular application to isogeometric design and analysis. Computer Methods in Applied Mechanics and Engineering, 284:55-105, February 2015.

[39] W. Wang, Y. Zhang, L. Liu, and T. J. R. Hughes. Trivariate solid T-spline construction from boundary triangulations with arbitrary genus topology. Computer-Aided Design, 45(2):351-360, February 2013.

[40] T. Warburton. An explicit construction of interpolation nodes on the simplex. Journal of Engineering Mathematics, 56(3):247-262, September 2006.

[41] S. Xia and X. Qian. Isogeometric analysis with Bézier tetrahedra. Computer Methods in Applied Mechanics and Engineering. 
[42] Z. Q. Xie, R. Sevilla, O. Hassan, and K. Morgan. The generation of arbitrary order curved meshes for 3d finite element analysis. Computational Mechanics, 51(3):361-374, June 2012.

[43] G. Xu, B. Mourrain, R. Duvigneau, and A. Galligo. Analysis-suitable volume parameterization of multi-block computational domain in isogeometric applications. Computer-Aided Design, 45(2):395-404, February 2013.

[44] S. Zeng and E. Cohen. Hybrid volume completion with higher-order Bézier elements. Computer Aided Geometric Design, 35-36:180-191, May 2015.

[45] A. Ženíšek. Polynomial approximation on tetrahedrons in the finite element method. Journal of Approximation Theory, 7(4):334-351, 1973. 